University of Texas Rio Grande Valley

ScholarWorks @ UTRGV

$11-2021$

\title{
Searches for Continuous Gravitational Waves from Young Supernova Remnants in the Early Third Observing Run of Advanced LIGO and Virgo
}

R. Abbott

T. D. Abbott

S. Abraham

Teviet Creighton

The University of Texas Rio Grande Valley

Mario C. Diaz

The University of Texas Rio Grande Valley

See next page for additional authors

Follow this and additional works at: https://scholarworks.utrgv.edu/pa_fac

Part of the Astrophysics and Astronomy Commons, and the Physics Commons

\section{Recommended Citation}

R. Abbott et al 2021 ApJ 92180.

This Article is brought to you for free and open access by the College of Sciences at ScholarWorks @ UTRGV. It has been accepted for inclusion in Physics and Astronomy Faculty Publications and Presentations by an authorized administrator of ScholarWorks @ UTRGV. For more information, please contact justin.white@utrgv.edu, william.flores01@utrgv.edu. 


\section{Authors}

R. Abbott, T. D. Abbott, S. Abraham, Teviet Creighton, Mario C. Diaz, Soma Mukherjee, Volker Quetschke, Karla E. Ramirez, and Wenhui Wang 


\section{Searches for Continuous Gravitational Waves from Young Supernova Remnants in the Early Third Observing Run of Advanced LIGO and Virgo}

R. Abbott ${ }^{1}$, T. D. Abbott ${ }^{2}$, S. Abraham ${ }^{3}$, F. Acernese ${ }^{4,5}$, K. Ackley ${ }^{6}$, A. Adams ${ }^{7}$, C. Adams ${ }^{8}$, R. X. Adhikari ${ }^{1}$, V. B. Adya ${ }^{9}$, C. Affeldt ${ }^{10,11}$, D. Agarwal ${ }^{3}$, M. Agathos ${ }^{12,13}$, K. Agatsuma ${ }^{14}$, N. Aggarwal ${ }^{15}$, O. D. Aguiar ${ }^{16}$, L. Aiello ${ }^{17,18,19}$, A. Ain ${ }^{20,21}$, P. Ajith ${ }^{22}$, T. Akutsu ${ }^{23,24}$, K. M. Aleman ${ }^{25}$, G. Allen ${ }^{26}$, A. Allocca, ${ }^{57}$, P. A. Altin ${ }^{9}$, A. Amato ${ }^{28}$, S. Anand ${ }^{1}$, A. Ananyeva ${ }^{1}$, S. B. Anderson ${ }^{1}$, W. G. Anderson ${ }^{29}$, M. Ando ${ }^{30,31}$, S. V. Angelova ${ }^{32}$, S. Ansoldi ${ }^{33,34}$, J. M. Antelis ${ }^{35}$, S. Antier ${ }^{36}$, S. Appert ${ }^{1}$,

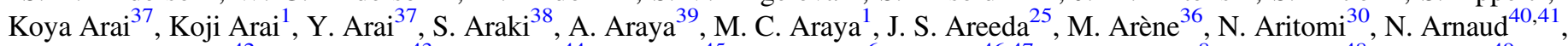

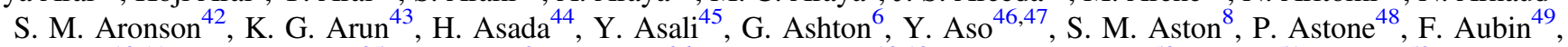
P. Aufmuth ${ }^{10,11}$, K. AultONeal ${ }^{35}$, C. Austin ${ }^{2}$, S. Babak ${ }^{36}$, F. Badaracco ${ }^{18,19}$, M. K. M. Bader ${ }^{50}$, S. Bae ${ }^{51}$, Y. Bae ${ }^{52}$, A. M. Baer ${ }^{7}$, S. Bagnasco ${ }^{53}$, Y. Bai ${ }^{1}$, L. Baiotti ${ }^{54}$, J. Baird ${ }^{36}$, R. Bajpai ${ }^{55}$, M. Ball ${ }^{56}$, G. Ballardin ${ }^{41}$, S. W. Ballmer ${ }^{57}$, M. Bals ${ }^{35}$, A. Balsamo ${ }^{7}$, G. Baltus $^{58}$, S. Banagiri ${ }^{59}$, D. Bankar ${ }^{3}$, R. S. Bankar ${ }^{3}$, J. C. Barayoga ${ }^{1}$, C. Barbieri ${ }^{60,61,62}$, B. C. Barish ${ }^{1}$, D. Barker ${ }^{63}$, P. Barneo $^{64}$, F. Barone ${ }^{5,65}$, B. Barr ${ }^{66}$, L. Barsotti ${ }^{67}$, M. Barsuglia ${ }^{36}$, D. Barta ${ }^{68}$, J. Bartlett ${ }^{63}$, M. A. Barton ${ }^{23,66}$, I. Bartos ${ }^{42}$, R. Bassiri ${ }^{69}$, A. Basti ${ }^{2021}$, M. Bawaj ${ }^{70,71}$, J. C. Bayley ${ }^{66}$, A. C. Baylor ${ }^{29}$, M. Bazzan ${ }^{72,73}$, B. Bécsy ${ }^{74}$, V. M. Bedakihale ${ }^{75}$, M. Bejger ${ }^{76}$, I. Belahcene $^{40}$, V. Benedetto ${ }^{77}$, D. Beniwal ${ }^{78}$, M. G. Benjamin ${ }^{35}$, T. F. Bennett ${ }^{79}$, J. D. Bentley ${ }^{14}$, M. BenYaala ${ }^{32}$, F. Bergamin ${ }^{10,11}$, B. K. Berger ${ }^{69}$, S. Bernuzzi ${ }^{13}$, D. Bersanetti ${ }^{80}$, A. Bertolini ${ }^{50}$, J. Betzwieser ${ }^{8}$, R. Bhandare ${ }^{81}$, A. V. Bhandari ${ }^{3}$, D. Bhattacharjee ${ }^{82}$, S. Bhaumik ${ }^{42}$, J. Bidler ${ }^{25}$, I. A. Bilenko ${ }^{83}$, G. Billingsley ${ }^{1}$, R. Birney ${ }^{84}$, O. Birnholtz ${ }^{85}$, S. Biscans ${ }^{1,67}$, M. Bischi ${ }^{86,87}$,

S. Biscoveanu ${ }^{67}$, A. Bisht ${ }^{10,11}$, B. Biswas ${ }^{3}$, M. Bitossi ${ }^{20,41}$, M.-A. Bizouard ${ }^{88}$, J. K. Blackburn ${ }^{1}$, J. Blackman ${ }^{89}$, C. D. Blair ${ }^{8,90}$, D. G. Blair ${ }^{90}$, R. M. Blair ${ }^{63}$, F. Bobba ${ }^{91,92}$, N. Bode ${ }^{10,11}$, M. Boer ${ }^{88}$, G. Bogaert ${ }^{88}$, M. Boldrini ${ }^{48,93}$, F. Bondu ${ }^{94}$, E. Bonilla ${ }^{69}$,

R. Bonnand ${ }^{49}$, P. Booker ${ }^{10,11}$, B. A. Boom ${ }^{50}$, R. Bork ${ }^{1}$, V. Boschi ${ }^{20}$, N. Bose ${ }^{95}$, S. Bose $^{3}$, V. Bossilkov ${ }^{90}$, V. Boudart ${ }^{58}$,

Y. Bouffanais $^{72,73}$, A. Bozzi ${ }^{41}$, C. Bradaschia ${ }^{20}$, P. R. Brady ${ }^{29}$, A. Bramley ${ }^{8}$, A. Branch ${ }^{8}$, M. Branchesi ${ }^{18,19}$, J. E. Brau ${ }^{56}$, M. Breschi ${ }^{13}$, T. Briant ${ }^{96}$, J. H. Briggs ${ }^{66}$, A. Brillet ${ }^{88}$, M. Brinkmann ${ }^{10,11}$, P. Brockill ${ }^{29}$, A. F. Brooks ${ }^{1}$, J. Brooks ${ }^{41}$, D. D. Brown ${ }^{78}$, S. Brunett ${ }^{1}$, G. Bruno ${ }^{97}$, R. Bruntz ${ }^{7}$, J. Bryant ${ }^{14}$, A. Buikema ${ }^{67}$, T. Bulik ${ }^{98}$, H. J. Bulten ${ }^{50,99}$, A. Buonanno ${ }^{100,101}$, R. Buscicchio ${ }^{14}$, D. Buskulic ${ }^{49}$, R. L. Byer ${ }^{69}$, L. Cadonati ${ }^{102}$, M. Caesar ${ }^{103}$, G. Cagnoli $^{28}$, C. Cahillane ${ }^{1}$, H. W. Cain III ${ }^{2}$, J. Calderón Bustillo ${ }^{104}$, J. D. Callaghan ${ }^{66}$, T. A. Callister ${ }^{105,106}$, E. Calloni ${ }^{5,27}$, J. B. Camp ${ }^{107}$, M. Canepa ${ }^{80,108}$, M. Cannavacciuolo ${ }^{91}$, K. C. Cannon ${ }^{31}$, H. Cao ${ }^{78}$, J. Cao ${ }^{109}$, Z. Cao ${ }^{110}$, E. Capocasa ${ }^{23}$, E. Capote ${ }^{25}$, G. Carapella ${ }^{9192}$, F. Carbognani ${ }^{41}$, J. B. Carlin ${ }^{111}$, M. F. Carney ${ }^{15}$, M. Carpinelli ${ }^{112,113}$, G. Carullo ${ }^{20,21}$, T. L. Carver ${ }^{17}$, J. Casanueva Diaz ${ }^{41}$, C. Casentini ${ }^{114,115}$, G. Castaldi ${ }^{116}$, S. Caudill ${ }^{50,117}$, M. Cavaglià ${ }^{82}$, F. Cavalier ${ }^{40}$, R. Cavalieri ${ }^{41}$, G. Cella ${ }^{20}$, P. Cerdá-Durán ${ }^{118}$, E. Cesarini ${ }^{115}$, W. Chaibi ${ }^{88}$, K. Chakravarti ${ }^{3}$, B. Champion ${ }^{119}$, C.-H. Chan ${ }^{120}$, C. Chan ${ }^{31}$, C. L. Chan ${ }^{104}$, M. Chan ${ }^{121}$, K. Chandra ${ }^{95}$, P. Chanial ${ }^{41}$, S. Chao ${ }^{120}$, P. Charlton ${ }^{122}$, E. A. Chase ${ }^{15}$, E. Chassande-Mottin ${ }^{36}$, D. Chatterjee ${ }^{29}$, M. Chaturvedi ${ }^{81}$, A. Chen ${ }^{104}$, C. Chen ${ }^{123,124}$, H. Y. Chen ${ }^{125}$, J. Chen ${ }^{120}$, K. Chen ${ }^{126}$, X. Chen ${ }^{90}$, Y.-B. Chen ${ }^{89}$, Y.-R. Chen ${ }^{124}$, Z. Chen ${ }^{17}$, H. Cheng ${ }^{42}$, C. K. Cheong ${ }^{104}$, H. Y. Cheung ${ }^{104}$, H. Y. Chia ${ }^{42}$, F. Chiadini ${ }^{92,127}$, C-Y. Chiang ${ }^{128}$, R. Chierici ${ }^{129}$, A. Chincarini ${ }^{80}$, M. L. Chiofalo ${ }^{20,21}$, A. Chiummo ${ }^{41}$, G. Cho ${ }^{130}$, H. S. Cho ${ }^{131}$ ', S. Choate ${ }^{103}$, R. K. Choudhary ${ }^{90}$, S. Choudhary ${ }^{3}$, N. Christensen ${ }^{88}$, H. Chu ${ }^{126}$, Q. Chu ${ }^{90}$, Y-K. Chu ${ }^{128}$, S. Chua ${ }^{96}$, K. W. Chung ${ }^{132}$, G. Ciani ${ }^{72,73}$, P. Ciecielag ${ }^{76}$, M. Cieślar ${ }^{76}$, M. Cifaldi ${ }^{14,115}$, A. A. Ciobanu ${ }^{78}$, R. Ciolfi ${ }^{73,133}$, F. Cipriano ${ }^{88}$, A. Cirone ${ }^{80,108}$, F. Clara ${ }^{63}$, E. N. Clark $^{134}$, J. A. Clark ${ }^{102}$, L. Clarke ${ }^{135}$, P. Clearwater ${ }^{111}$, S. Clesse ${ }^{136}$, F. Cleva ${ }^{88}$, E. Coccia ${ }^{18,19}$, P.-F. Cohadon ${ }^{96}$, D. E. Cohen ${ }^{40}$, L. Cohen ${ }^{2}$, M. Colleoni ${ }^{137}$, C. G. Collette ${ }^{138}$, M. Colpi ${ }^{60,61}$, C. M. Compton ${ }^{63}$, M. Constancio Jr. ${ }^{16}$, L. Conti ${ }^{73}$, S. J. Cooper ${ }^{14}$, P. Corban ${ }^{8}$, T. R. Corbitt ${ }^{2}$, I. Cordero-Carrión ${ }^{139}$, S. Corezzi ${ }^{70,71}$, K. R. Corley ${ }^{45}$, N. Cornish ${ }^{74}$, D. Corre ${ }^{40}$, A. Corsi ${ }^{140}$, S. Cortese ${ }^{41}$, C. A. Costa ${ }^{16}$, R. Cotesta ${ }^{101}$, M. W. Coughlin ${ }^{59}$, S. B. Coughlin ${ }^{15,17}$, J.-P. Coulon ${ }^{88}$, S. T. Countryman ${ }^{45}$, B. Cousins ${ }^{141}$, P. Couvares ${ }^{1}$, P. B. Covas ${ }^{137}$, D. M. Coward $^{90}$, M. J. Cowart ${ }^{8}$, D. C. Coyne ${ }^{1}$, R. Coyne ${ }^{142}$, J. D. E. Creighton ${ }^{29}$,

T. D. Creighton ${ }^{143}$, A. W. Criswell ${ }^{59}$, M. Croquette $^{96}$, S. G. Crowder $^{144}$, J. R. Cudell ${ }^{58}$, T. J. Cullen ${ }^{2}$, A. Cumming ${ }^{66}$,

R. Cummings ${ }^{66}$, E. Cuoco ${ }^{20,41,145}$, M. Curyło ${ }^{98}$, T. Dal Canton ${ }^{40,101}$, G. Dálya ${ }^{146}$, A. Dana ${ }^{69}$, L. M. DaneshgaranBajastani ${ }^{79}$, B. D’Angelo ${ }^{80,108}$, S. L. Danilishin ${ }^{147}$, S. D'Antonio ${ }^{115}$, K. Danzmann ${ }^{10,11}$, C. Darsow-Fromm ${ }^{148}$, A. Dasgupta ${ }^{75}$,

L. E. H. Datrier ${ }^{66}$, V. Dattilo ${ }^{41}$, I. Dave ${ }^{81}$, M. Davier ${ }^{40}$, G. S. Davies ${ }^{149,150}$, D. Davis ${ }^{1}$, E. J. Daw ${ }^{151}$, R. Dean ${ }^{103}$, D. DeBra ${ }^{69}$,

M. Deenadayalan ${ }^{3}$, J. Degallaix ${ }^{152}$, M. De Laurentis ${ }^{5,27}$, S. Deléglise ${ }^{96}$, V. Del Favero ${ }^{119}$, F. De Lillo9 ${ }^{97}$, N. De Lillo ${ }^{66}$,

W. Del Pozzo ${ }^{20,21}$, L. M. DeMarchi ${ }^{15}$, F. De Matteis ${ }^{114,115}$, V. D’Emilio ${ }^{17}$, N. Demos ${ }^{67}$, T. Dent ${ }^{149}$, A. Depasse ${ }^{97}$,

R. De Pietri ${ }^{153,154}$, R. De Rosa ${ }^{5,27}$, C. De Rossi ${ }^{41}$, R. DeSalvo ${ }^{116}$, R. De Simone ${ }^{127}$, S. Dhurandhar ${ }^{3}$, M. C. Díaz ${ }^{143}$, M. Diaz-Ortiz Jr. ${ }^{42}$, N. A. Didio ${ }^{57}$, T. Dietrich ${ }^{101}$, L. Di Fiore ${ }^{5}$, C. Di Fronzo ${ }^{14}$, C. Di Giorgio ${ }^{91,92}$, F. Di Giovanni ${ }^{118}$, T. Di Girolamo ${ }^{5,27}$, A. Di Lieto ${ }^{20,21}$, B. Ding ${ }^{138}$, S. Di Pace ${ }^{48,93}$, I. Di Palma ${ }^{48,93}$, F. Di Renzo ${ }^{20,21}$, A. K. Divakarla ${ }^{42}$, A. Dmitriev ${ }^{14}$, Z. Doctor ${ }^{56}$, L. D’Onofrio ${ }^{5,27}$, F. Donovan ${ }^{67}$, K. L. Dooley ${ }^{17}$, S. Doravari ${ }^{3}$, I. Dorrington ${ }^{17}$, M. Drago ${ }^{18,19}$, J. C. Driggers ${ }^{63}$, Y. Drori ${ }^{1}$, Z. Du ${ }^{109}$, J.-G. Ducoin ${ }^{40}$, P. Dupej ${ }^{66}$, O. Durante ${ }^{91,92}$, D. D’Urso ${ }^{112,113}$, P.-A. Duverne ${ }^{40}$, S. E. Dwyer ${ }^{63}$, P. J. Easter ${ }^{6}$, M. Ebersold ${ }^{155}$, G. Eddolls ${ }^{66}$, B. Edelman ${ }^{56}$, T. B. Edo ${ }^{1,151}$, O. Edy ${ }^{150}$, A. Effler ${ }^{8}$, S. Eguchi ${ }^{121}$, J. Eichholz ${ }^{9}$, S. S. Eikenberry ${ }^{42}$, M. Eisenmann ${ }^{49}$, R. A. Eisenstein ${ }^{67}$, A. Ejlli1 ${ }^{17}$, Y. Enomoto ${ }^{30}$, L. Errico ${ }^{5,27}$, R. C. Essick $^{125}$, H. Estellés ${ }^{137}$, D. Estevez ${ }^{156}$, Z. Etienne ${ }^{157}$, T. Etzel ${ }^{1}$, M. Evans ${ }^{67}$, T. M. Evans ${ }^{8}$, B. E. Ewing ${ }^{141}$, V. Fafone ${ }^{18,114,115}$, H. Fair ${ }^{57}$, S. Fairhurst ${ }^{17}$, X. Fan ${ }^{109}$, A. M. Farah ${ }^{125}$, S. Farinon ${ }^{80}$, B. Farr ${ }^{56}$, W. M. Farr ${ }^{105,106}$, N. W. Farrow ${ }^{6}$, E. J. Fauchon-Jones ${ }^{17}$, M. Favata ${ }^{158}$, M. Fays ${ }^{58,151}$, M. Fazio ${ }^{159}$, J. Feicht ${ }^{1}$, M. M. Fejer ${ }^{69}$, F. Feng ${ }^{36}$, E. Fenyvesi ${ }^{68,160}$, D. L. Ferguson ${ }^{102}$, A. Fernandez-Galiana ${ }^{67}$, 
I. Ferrante ${ }^{20,21}$, T. A. Ferreira ${ }^{16}$, F. Fidecaro ${ }^{20,21}$, P. Figura ${ }^{98}$, I. Fiori ${ }^{41}$, M. Fishbach ${ }^{15,125}$, R. P. Fisher ${ }^{7}$, R. Fittipaldi ${ }^{92,161}$, V. Fiumara ${ }^{92,162}$, R. Flaminio ${ }^{23,49}$, E. Floden ${ }^{59}$, E. Flynn ${ }^{25}$, H. Fong ${ }^{31}$, J. A. Font ${ }^{118,163}$, B. Fornal ${ }^{164}$, P. W. F. Forsyth ${ }^{9}$, A. Franke ${ }^{148}$, S. Frasca ${ }^{48,93}$, F. Frasconi ${ }^{20}$, C. Frederick ${ }^{165}$, Z. Frei ${ }^{146}$, A. Freise ${ }^{166}$, R. Frey ${ }^{56}$, P. Fritschel ${ }^{67}$, V. V. Frolov ${ }^{8}$, G. G. Fronzé ${ }^{53}$, Y. Fujii ${ }^{167}$, Y. Fujikawa ${ }^{168}$, M. Fukunaga ${ }^{37}$, M. Fukushima ${ }^{24}$, P. Fulda ${ }^{42}$, M. Fyffe ${ }^{8}$, H. A. Gabbard ${ }^{66}$, B. U. Gadre ${ }^{101}$, S. M. Gaebel ${ }^{14}$, J. R. Gair ${ }^{101}$, J. Gais ${ }^{104}$, S. Galaudage ${ }^{6}$, R. Gamba ${ }^{13}$, D. Ganapathy ${ }^{67}$, A. Ganguly ${ }^{22}$, D. Gao ${ }^{169}$, S. G. Gaonkar ${ }^{3}$, B. Garaventa ${ }^{80,108}$, C. García-Núñez ${ }^{84}$, C. García-Quirós ${ }^{137}$, F. Garufi ${ }^{5,27}$, B. Gateley ${ }^{63}$, S. Gaudio $^{35}$, V. Gayathri ${ }^{42}$, G. Ge ${ }^{169}$, G. Gemme ${ }^{80}$, A. Gennai ${ }^{20}$, J. George ${ }^{81}$, L. Gergely ${ }^{170}$, P. Gewecke ${ }^{148}$, S. Ghonge ${ }^{102}$, Abhirup. Ghosh ${ }^{101}$, Archisman Ghosh ${ }^{171}$, Shaon Ghosh ${ }^{29,158}$, Shrobana Ghosh ${ }^{17}$, Sourath Ghosh ${ }^{42}$, B. Giacomazzo ${ }^{60,61,62}$, L. Giacoppo ${ }^{48,93}$, J. A. Giaime ${ }^{2,8}$, K. D. Giardina ${ }^{8}$, D. R. Gibson ${ }^{84}$, C. Gier ${ }^{32}$, M. Giesler ${ }^{89}$, P. Giri ${ }^{20,21}$, F. Gissi ${ }^{77}$, J. Glanzer ${ }^{2}$, A. E. Gleckl ${ }^{25}$, P. Godwin ${ }^{141}$, E. Goetz ${ }^{172}$, R. Goetz ${ }^{42}$, N. Gohlke ${ }^{10,11}$, B. Goncharov ${ }^{6}$, G. González ${ }^{2}$, A. Gopakumar ${ }^{173}$, M. Gosselin ${ }^{41}$, R. Gouaty ${ }^{49}$, B. Grace ${ }^{9}$, A. Grado ${ }^{5,174}$, M. Granata ${ }^{152}$, V. Granata ${ }^{91}$, A. Grant ${ }^{66}$, S. Gras ${ }^{67}$, P. Grassia ${ }^{1}$, C. Gray ${ }^{63}$, R. Gray ${ }^{66}$, G. Greco $^{70}$, A. C. Green ${ }^{42}$, R. Green ${ }^{17}$, A. M. Gretarsson ${ }^{35}$, E. M. Gretarsson ${ }^{35}$, D. Griffith ${ }^{1}$, W. Griffiths ${ }^{17}$, H. L. Griggs ${ }^{102}$, G. Grignani $^{70,71}$, A. Grimaldi ${ }^{175,176}$, E. Grimes ${ }^{35}$, S. J. Grimm ${ }^{18,19}$, H. Grote ${ }^{17}$, S. Grunewald ${ }^{101}$, P. Gruning ${ }^{40}$, J. G. Guerrero ${ }^{25}$, G. M. Guidi ${ }^{86,87}$, A. R. Guimaraes ${ }^{2}$, G. Guixé ${ }^{64}$, H. K. Gulati ${ }^{75}$, H.-K. Guo ${ }^{164}$, Y. Guo ${ }^{50}$, Anchal Gupta ${ }^{1}$, Anuradha Gupta ${ }^{177}$, P. Gupta ${ }^{50,117}$, E. K. Gustafson ${ }^{1}$, R. Gustafson ${ }^{178}$, F. Guzman ${ }^{134}$, S. Ha ${ }^{179}$, L. Haegel ${ }^{36}$, A. Hagiwara ${ }^{37,180}$, S. Haino ${ }^{128}$,

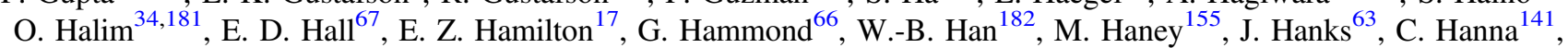

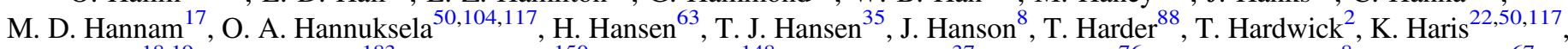
J. Harms ${ }^{18,19}$, G. M. Harry ${ }^{183}$, I. W. Harry ${ }^{150}$, D. Hartwig ${ }^{148}$, K. Hasegawa ${ }^{37}$, B. Haskell ${ }^{76}$, R. K. Hasskew ${ }^{8}$, C.-J. Haster ${ }^{67}$,

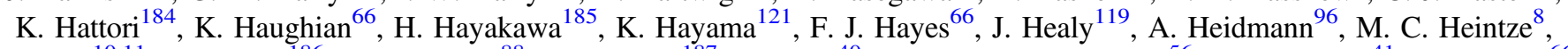
J. Heinze ${ }^{10,11}$, J. Heinzel ${ }^{186}$, H. Heitmann ${ }^{88}$, F. Hellman ${ }^{187}$, P. Hello ${ }^{40}$, A. F. Helmling-Cornell ${ }^{56}$, G. Hemming ${ }^{41}$, M. Hendry ${ }^{66}$, I. S. Heng $^{66}$, E. Hennes ${ }^{50}$, J. Hennig ${ }^{10,11}$, M. H. Hennig ${ }^{10,11}$, F. Hernandez Vivanco ${ }^{6}$, M. Heurs ${ }^{10,11}$, S. Hild ${ }^{50,147}$, P. Hill ${ }^{32}$, Y. Himemoto $^{188}$, A. S. Hines ${ }^{134}$, Y. Hiranuma ${ }^{189}$, N. Hirata ${ }^{23}$, E. Hirose ${ }^{37}$, S. Hochheim ${ }^{10,11}$, D. Hofman ${ }^{152}$, J. N. Hohmann ${ }^{148}$, A. M. Holgado ${ }^{26}$, N. A. Holland ${ }^{9}$, I. J. Hollows ${ }^{151}$, Z. J. Holmes ${ }^{78}$, K. Holt ${ }^{8}$, D. E. Holz ${ }^{125}$, Z. Hong ${ }^{190}$, P. Hopkins ${ }^{17}$, J. Hough ${ }^{66}$, E. J. Howell ${ }^{90}$, C. G. Hoy $^{17}$, D. Hoyland ${ }^{14}$, A. Hreibi ${ }^{10,11}$, B-H. Hsieh ${ }^{37}$, Y. Hsu ${ }^{120}$, G-Z. Huang ${ }^{190}$, H-Y. Huang ${ }^{128}$, P. Huang ${ }^{169}$, Y-C. Huang $^{124}$, Y.-J. Huang ${ }^{128}$, Y.-W. Huang ${ }^{67}$, M. T. Hübner ${ }^{6}$, A. D. Huddart ${ }^{135}$, E. A. Huerta ${ }^{26}$, B. Hughey ${ }^{35}$, D. C. Y. Hui ${ }^{191}$, V. Hui ${ }^{49}$, S. Husa ${ }^{137}$, S. H. Huttner ${ }^{66}$, R. Huxford ${ }^{141}$, T. Huynh-Dinh ${ }^{8}$, S. Ide ${ }^{192}$, B. Idzkowski ${ }^{98}$, A. Iess ${ }^{14,115}$, B. Ikenoue ${ }^{24}$, S. Imam ${ }^{190}$, K. Inayoshi ${ }^{193}$, H. Inchauspe ${ }^{42}$, C. Ingram ${ }^{78}$, Y. Inoue ${ }^{126}$, G. Intini ${ }^{48,93}$, K. Ioka ${ }^{194}$, M. Isi ${ }^{67}$, K. Isleif ${ }^{148}$, K. Ito ${ }^{195}$, Y. Itoh ${ }^{196,197}$, B. R. Iyer ${ }^{22}$, K. Izumi ${ }^{198}$, V. JaberianHamedan ${ }^{90}$, T. Jacqmin ${ }^{96}$, S. J. Jadhav ${ }^{199}$, S. P. Jadhav ${ }^{3}$, A. L. James ${ }^{17}$, A. Z. Jan ${ }^{119}$, K. Jani ${ }^{102}$, K. Janssens ${ }^{200}$, N. N. Janthalur ${ }^{199}$, P. Jaranowski ${ }^{201}$, D. Jariwala ${ }^{42}$, R. Jaume ${ }^{137}$, A. C. Jenkins ${ }^{132}$, C. Jeon ${ }^{202}$, M. Jeunon ${ }^{59}$, W. Jia ${ }^{67}$, J. Jiang ${ }^{42}$, H.-B. Jin ${ }^{203,204}$, G. R. Johns ${ }^{7}$, A. W. Jones ${ }^{90}$, D. I. Jones ${ }^{205}$, J. D. Jones ${ }^{63}$, P. Jones ${ }^{14}$, R. Jones ${ }^{66}$, R. J. G. Jonker ${ }^{50}$, L. Ju ${ }^{90}$, K. Jung ${ }^{179}$, P. Jung ${ }^{185}$, J. Junker ${ }^{10,11}$, K. Kaihotsu ${ }^{195}$, T. Kajita ${ }^{206}$, M. Kakizaki ${ }^{184}$,

C. V. Kalaghatgi ${ }^{17}$, V. Kalogera ${ }^{15}$, B. Kamai ${ }^{1}$, M. Kamiizumi ${ }^{185}$, N. Kanda ${ }^{196,197}$, S. Kandhasamy ${ }^{3}$, G. Kang ${ }^{51}$, J. B. Kanner ${ }^{1}$, Y. Kao ${ }^{120}$, S. J. Kapadia ${ }^{22}$, D. P. Kapasi ${ }^{9}$, S. Karat ${ }^{1}$, C. Karathanasis ${ }^{207}$, S. Karki ${ }^{82}$, R. Kashyap ${ }^{141}$, M. Kasprzack ${ }^{1}$,

W. Kastaun ${ }^{10,11}$, S. Katsanevas ${ }^{41}$, E. Katsavounidis ${ }^{67}$, W. Katzman ${ }^{8}$, T. Kaur ${ }^{90}$, K. Kawabe ${ }^{63}$, K. Kawaguchi ${ }^{37}$, N. Kawai ${ }^{208}$, T. Kawasaki ${ }^{30}$, F. Kéfélian ${ }^{88}$, D. Keitel ${ }^{137}$, J. S. Key ${ }^{209}$, S. Khadka ${ }^{69}$, F. Y. Khalili ${ }^{83}$, I. Khan ${ }^{18,115}$, S. Khan ${ }^{17}$, E. A. Khazanov ${ }^{210}$, N. Khetan ${ }^{18,19}$, M. Khursheed ${ }^{81}$, N. Kijbunchoo ${ }^{9}$, C. Kim ${ }^{202,211}$, J. C. Kim $^{212}$, J. Kim ${ }^{213}$, K. Kim ${ }^{214}$, W. S. Kim ${ }^{52}$, Y.-M. Kim ${ }^{179}$, C. Kimball ${ }^{15}$, N. Kimura ${ }^{180}$, P. J. King ${ }^{63}$, M. Kinley-Hanlon ${ }^{66}$, R. Kirchhoff ${ }^{10,11}$, J. S. Kissel ${ }^{63}$, N. Kita ${ }^{30}$, H. Kitazawa ${ }^{195}$, L. Kleybolte ${ }^{148}$, S. Klimenko ${ }^{42}$, A. M. Knee ${ }^{172}$, T. D. Knowles ${ }^{157}$, E. Knyazev ${ }^{67}$, P. Koch ${ }^{10,11}$, G. Koekoek ${ }^{50,147}$, Y. Kojima ${ }^{215}$, K. Kokeyama ${ }^{185}$, S. Koley ${ }^{50}$, P. Kolitsidou ${ }^{17}$, M. Kolstein ${ }^{207}$, K. Komori ${ }^{30,67}$, V. Kondrashov ${ }^{1}$, A. K. H. Kong ${ }^{124}$, A. Kontos ${ }^{216}$, N. Koper ${ }^{10,11}$, M. Korobko ${ }^{148}$, K. Kotake ${ }^{121}$, M. Kovalam ${ }^{90}$, D. B. Kozak ${ }^{1}$, C. Kozakai ${ }^{46}$, R. Kozu ${ }^{217}$, V. Kringel ${ }^{10,11}$, N. V. Krishnendu ${ }^{10,11}$, A. Królak ${ }^{218,219}$, G. Kuehn ${ }^{10,11}$, F. Kuei ${ }^{120}$, A. Kumar ${ }^{199}$, P. Kumar ${ }^{220}$, Rahul Kumar ${ }^{63}$, Rakesh Kumar ${ }^{75}$, J. Kume $^{31}$, K. Kuns ${ }^{67}$, C. Kuo ${ }^{126}$, H-S. Kuo ${ }^{190}$, Y. Kuromiya ${ }^{195}$, S. Kuroyanagi ${ }^{221}$, K. Kusayanagi ${ }^{208}$, K. Kwak ${ }^{179}$, S. Kwang ${ }^{29}$, D. Laghi ${ }^{20,21}$, E. Lalande ${ }^{222}$, T. L. Lam ${ }^{104}$, A. Lamberts ${ }^{88,223}$, M. Landry ${ }^{63}$, B. B. Lane ${ }^{67}$, R. N. Lang ${ }^{67}$, J. Lange ${ }^{119,224}$, B. Lantz ${ }^{69}$, I. La Rosa ${ }^{49}$, A. Lartaux-Vollard ${ }^{40}$, P. D. Lasky ${ }^{6}$, M. Laxen ${ }^{8}$, A. Lazzarini ${ }^{1}$, C. Lazzaro ${ }^{72,73}$, P. Leaci ${ }^{48,93}$, S. Leavey ${ }^{10,11}$, Y. K. Lecoeuche ${ }^{63}$, H. K. Lee ${ }^{225}$, H. M. Lee ${ }^{214}$, H. W. Lee ${ }^{212}$, J. Lee ${ }^{130}$, K. Lee ${ }^{69}$, R. Lee ${ }^{124}$, J. Lehmann ${ }^{10,11}$, A. Lemaître ${ }^{226}$, E. Leon ${ }^{25}$, M. Leonardi ${ }^{23}$, N. Leroy ${ }^{40}$, N. Letendre ${ }^{49}$, Y. Levin ${ }^{6}$, J. N. Leviton ${ }^{178}$, A. K. Y. Li ${ }^{1}$, B. Li ${ }^{120}$, J. Li $^{15}$, K. L. Li $^{124}$, T. G. F. Li $^{104}$, X. Li ${ }^{89}$, C-Y. Lin ${ }^{227}$, F-K. Lin ${ }^{128}$, F-L. Lin ${ }^{190}$, H. L. Lin ${ }^{126}$, L. C.-C. Lin ${ }^{179}$, F. Linde ${ }^{50,228}$, S. D. Linker ${ }^{79}$, J. N. Linley ${ }^{66}$, T. B. Littenberg ${ }^{229}$, G. C. Liu ${ }^{123}$, J. Liu ${ }^{10,11}$, K. Liu ${ }^{120}$, X. Liu ${ }^{29}$, M. Llorens-Monteagudo ${ }^{118}$, R. K. L. Lo ${ }^{1}$, A. Lockwood $^{230}$, M. L. Lollie ${ }^{2}$, L. T. London ${ }^{67}$, A. Longo ${ }^{231,232}$, D. Lopez ${ }^{155}$, M. Lorenzini ${ }^{114,115}{ }^{2}$, V. Loriette ${ }^{233}$, M. Lormand ${ }^{8}$, G. Losurdo $^{20}$, J. D. Lough ${ }^{10,11}$, C. O. Lousto ${ }^{119}$, G. Lovelace ${ }^{25}$, H. Lück ${ }^{10,11}$, D. Lumaca ${ }^{114,115}$, A. P. Lundgren ${ }^{150}$, L.-W. Luo ${ }^{128}$, R. Macas ${ }^{17}$, M. MacInnis ${ }^{67}$, D. M. Macleod ${ }^{17}$, I. A. O. MacMillan ${ }^{1}$, A. Macquet ${ }^{88}$, I. Magaña Hernandez ${ }^{29}$, F. Magaña-Sandoval ${ }^{42}$, C. Magazzù ${ }^{20}$, R. M. Magee ${ }^{141}$, R. Maggiore ${ }^{14}$, E. Majorana ${ }^{48,93}$, C. Makarem ${ }^{1}$, I. Maksimovic ${ }^{233}$, S. Maliakal ${ }^{1}$, A. Malik ${ }^{81}$,

N. Man $^{88}$, V. Mandic ${ }^{59}$, V. Mangano ${ }^{48,93}$, J. L. Mango ${ }^{234}$, G. L. Mansell ${ }^{63,67}$, M. Manske ${ }^{29}$, M. Mantovani ${ }^{41}$, M. Mapelli ${ }^{72,73}$,

F. Marchesoni ${ }^{70,235}$, M. Marchio ${ }^{23}$, F. Marion ${ }^{49}$, Z. Mark ${ }^{89}$, S. Márka ${ }^{45}$, Z. Márka ${ }^{45}$, C. Markakis ${ }^{12}$, A. S. Markosyan ${ }^{69}$,

A. Markowitz ${ }^{1}$, E. Maros ${ }^{1}$, A. Marquina ${ }^{139}$, S. Marsat ${ }^{36}$, F. Martelli ${ }^{86,87}$, I. W. Martin ${ }^{66}$, R. M. Martin ${ }^{158}$, M. Martinez ${ }^{207}$, V. Martinez $^{28}$, K. Martinovic ${ }^{132}$, D. V. Martynov ${ }^{14}$, E. J. Marx ${ }^{67}$, H. Masalehdan ${ }^{148}$, K. Mason ${ }^{67}$, E. Massera ${ }^{151}$, A. Masserot ${ }^{49}$, T. J. Massinger ${ }^{67}$, M. Masso-Reid ${ }^{66}$, S. Mastrogiovanni ${ }^{36}$, A. Matas ${ }^{101}$, M. Mateu-Lucena ${ }^{137}$, F. Matichard ${ }^{1,67}$, 
M. Matiushechkina ${ }^{10,11}$, N. Mavalvala ${ }^{67}$, J. J. McCann ${ }^{90}$, R. McCarthy ${ }^{63}$, D. E. McClelland ${ }^{9}$, P. McClincy ${ }^{141}$, S. McCormick ${ }^{8}$, L. McCuller ${ }^{67}$, G. I. McGhee ${ }^{66}$, S. C. McGuire ${ }^{236}$, C. McIsaac ${ }^{150}$, J. McIver ${ }^{172}$, D. J. McManus ${ }^{9}$, T. McRae ${ }^{9}$, S. T. McWilliams ${ }^{157}$, D. Meacher ${ }^{29}$, M. Mehmet ${ }^{10,11}$, A. K. Mehta ${ }^{101}$, A. Melatos ${ }^{111}$, D. A. Melchor ${ }^{25}$, G. Mendell ${ }^{63}$, A. Menendez-Vazquez ${ }^{207}$,

C. S. Menoni ${ }^{159}$, R. A. Mercer ${ }^{29}$, L. Mereni ${ }^{152}$, K. Merfeld ${ }^{56}$, E. L. Merilh ${ }^{63}$, J. D. Merritt ${ }^{56}$, M. Merzougui ${ }^{88}$, S. Meshkov ${ }^{1,284}$, C. Messenger ${ }^{66}$, C. Messick ${ }^{24}$, P. M. Meyers ${ }^{111}$, F. Meylahn ${ }^{10,11}$, A. Mhaske ${ }^{3}$, A. Miani ${ }^{175,176}$, H. Miao ${ }^{14}$, I. Michaloliakos ${ }^{42}$, C. Michel ${ }^{152}$, Y. Michimura ${ }^{30}$, H. Middleton ${ }^{111}$, L. Milano ${ }^{27}$, A. L. Miller ${ }^{42,97}$, M. Millhouse ${ }^{111}$, J. C. Mills ${ }^{17}$, E. Milotti ${ }^{34,181}$, M. C. Milovich-Goff ${ }^{79}$, O. Minazzoli ${ }^{88,237}$, Y. Minenkov ${ }^{115}$, N. Mio ${ }^{238}$, Ll. M. Mir ${ }^{207}$, A. Mishkin ${ }^{42}$, C. Mishra ${ }^{239}$, T. Mishra ${ }^{42}$, T. Mistry ${ }^{151}$, S. Mitra ${ }^{3}$, V. P. Mitrofanov ${ }^{83}$, G. Mitselmakher ${ }^{42}$, R. Mittleman ${ }^{67}$, O. Miyakawa ${ }^{185}$, A. Miyamoto ${ }^{196}$, Y. Miyazaki ${ }^{30}$, K. Miyo ${ }^{185}$, S. Miyoki ${ }^{185}$, Geoffrey Mo ${ }^{67}$, K. Mogushi ${ }^{82}$, S. R. P. Mohapatra ${ }^{67}$, S. R. Mohite ${ }^{29}$, I. Molina ${ }^{25}$, M. Molina-Ruiz ${ }^{187}$, M. Mondin ${ }^{79}$, M. Montani ${ }^{86,87}$, C. J. Moore ${ }^{14}$, D. Moraru ${ }^{63}$, F. Morawski ${ }^{76}$, A. More ${ }^{3}$, C. Moreno ${ }^{35}$, G. Moreno $^{63}$, Y. Mori ${ }^{195}$, S. Morisaki ${ }^{31,37}$, Y. Moriwaki ${ }^{184}$, B. Mours ${ }^{156}$, C. M. Mow-Lowry ${ }^{14}$, S. Mozzon ${ }^{150}$, F. Muciaccia ${ }^{48,93}$, Arunava Mukherjee ${ }^{66,240}$, D. Mukherjee ${ }^{141}$, Soma Mukherjee ${ }^{143}$, Subroto Mukherjee ${ }^{75}$, N. Mukund ${ }^{10,11}$, A. Mullavey ${ }^{8}$, J. Munch ${ }^{78}$, E. A. Muñiz ${ }^{57}$,

P. G. Murray ${ }^{66}$, R. Musenich ${ }^{80,108}$, S. L. Nadji ${ }^{10,11}$, K. Nagano ${ }^{198}$, S. Nagano ${ }^{241}$, A. Nagar ${ }^{53,242}$, K. Nakamura ${ }^{23}$, H. Nakano ${ }^{243}$, M. Nakano ${ }^{37}$, R. Nakashima ${ }^{208}$, Y. Nakayama ${ }^{184}$, I. Nardecchia ${ }^{114,115}$, T. Narikawa ${ }^{37}$, L. Naticchioni ${ }^{48}$, B. Nayak ${ }^{79}$,

R. K. Nayak $^{244}$, R. Negishi ${ }^{189}$, B. F. Neil ${ }^{90}$, J. Neilson ${ }^{77,92}$, G. Nelemans ${ }^{245}$, T. J. N. Nelson ${ }^{8}$, M. Nery ${ }^{10,11}$, A. Neunzert ${ }^{209}$, K. Y. $\mathrm{Ng}^{67}$, S. W. S. Ng ${ }^{78}$, C. Nguyen ${ }^{36}$, P. Nguyen $^{56}$, T. Nguyen ${ }^{67}$, L. Nguyen Quynh ${ }^{246}$, W.-T. Ni ${ }^{169,203,247}$, S. A. Nichols ${ }^{2}$, A. Nishizawa ${ }^{31}$, S. Nissanke ${ }^{50,248}$, F. Nocera ${ }^{41}$, M. Noh ${ }^{172}$, M. Norman ${ }^{17}$, C. North ${ }^{17}$, S. Nozaki ${ }^{184}$, L. K. Nuttall ${ }^{150}$, J. Oberling ${ }^{63}$, B. D. O’Brien ${ }^{42}$, Y. Obuchi ${ }^{24}$, J. O’Dell ${ }^{135}$, W. Ogaki ${ }^{37}$, G. Oganesyan ${ }^{18,19}$, J. J. Oh ${ }^{52}$, K. Oh $^{191}$, S. H. Oh $^{52}$, M. Ohashi ${ }^{185}$, N. Ohishi ${ }^{46}$, M. Ohkawa ${ }^{168}$, F. Ohme ${ }^{10,11}$, H. Ohta ${ }^{31}$, M. A. Okada ${ }^{16}$, Y. Okutani ${ }^{192}$, K. Okutomi ${ }^{185}$, C. Olivetto ${ }^{41}$, K. Oohara ${ }^{189}$, C. Ooi $^{30}$, R. Oram ${ }^{8}$, B. O'Reilly ${ }^{8}$, R. G. Ormiston ${ }^{59}$, N. D. Ormsby ${ }^{7}$, L. F. Ortega ${ }^{42}$, R. O'Shaughnessy ${ }^{119}$, E. O’Shea ${ }^{220}$,

S. Oshino ${ }^{185}$, S. Ossokine ${ }^{101}$, C. Osthelder ${ }^{1}$, S. Otabe ${ }^{208}$, D. J. Ottaway ${ }^{78}$, H. Overmier ${ }^{8}$, A. E. Pace ${ }^{141}$, G. Pagano ${ }^{20,21}$, M. A. Page ${ }^{90}$, G. Pagliaroli ${ }^{18,19}$, A. Pai ${ }^{95}$, S. A. Pai ${ }^{81}$, J. R. Palamos ${ }^{56}$, O. Palashov ${ }^{210}$, C. Palomba ${ }^{48}$, K. Pan ${ }^{124}$, P. K. Panda ${ }^{199}$, H. Pang ${ }^{126}$, P. T. H. Pang ${ }^{50,117}$, C. Pankow ${ }^{15}$, F. Pannarale ${ }^{48,93}$, B. C. Pant ${ }^{81}$, F. Paoletti ${ }^{20}$, A. Paoli ${ }^{41}$, A. Paolone ${ }^{48,249}$, A. Parisi ${ }^{123}$, J. Park ${ }^{214}$, W. Parker ${ }^{8,236}$, D. Pascucci ${ }^{50}$, A. Pasqualetti ${ }^{41}$, R. Passaquieti ${ }^{20,21}$, D. Passuello ${ }^{20}$, M. Patel ${ }^{7}$, B. Patricelli ${ }^{20,41}$, E. Payne ${ }^{6}$,

T. C. Pechsiri ${ }^{42}$, M. Pedraza ${ }^{1}$, M. Pegoraro ${ }^{73}$, A. Pele ${ }^{8}$, F. E. Peña Arellano ${ }^{185}$, S. Penn ${ }^{250}$, A. Perego ${ }^{175,176}$, A. Pereira ${ }^{28}$, T. Pereira $^{251}$, C. J. Perez ${ }^{63}$, C. Périgois ${ }^{49}$, A. Perreca ${ }^{175,176}$, S. Perriès ${ }^{129}$, J. Petermann ${ }^{148}$, D. Petterson ${ }^{1}$, H. P. Pfeiffer ${ }^{101}{ }^{1}$, K. A. Pham ${ }^{59}$, K. S. Phukon ${ }^{3,50,228}$, O. J. Piccinni ${ }^{48}$, M. Pichot ${ }^{88}$, M. Piendibene ${ }^{20,21}$, F. Piergiovanni ${ }^{86,87}$, L. Pierini ${ }^{48,93}$, V. Pierro ${ }^{77,92}$, G. Pillant ${ }^{41}$, F. Pilo ${ }^{20}$, L. Pinard ${ }^{152}$, I. M. Pinto ${ }^{77,92,252,253}$, B. J. Piotrzkowski ${ }^{29}$, K. Piotrzkowski ${ }^{97}$, M. Pirello ${ }^{63}$, M. Pitkin ${ }^{254}$, E. Placidi ${ }^{48,93}$, W. Plastino ${ }^{231,232}$, C. Pluchar ${ }^{134}$, R. Poggiani ${ }^{20,21}$, E. Polini ${ }^{49}$, D. Y. T. Pong ${ }^{104}$, S. Ponrathnam ${ }^{3}$, P. Popolizio $^{41}$, E. K. Porter ${ }^{36}$, J. Powell ${ }^{255}$, M. Pracchia ${ }^{49}$, T. Pradier ${ }^{156}$, A. K. Prajapati ${ }^{75}$, K. Prasai ${ }^{69}$, R. Prasanna ${ }^{199}$, G. Pratten ${ }^{14}$, T. Prestegard ${ }^{29}$, M. Principe ${ }^{77,92,252}$, G. A. Prodi ${ }^{176,256}$, L. Prokhorov ${ }^{14}$, P. Prosposito ${ }^{114,115}$, L. Prudenzi $^{101}$, A. Puecher ${ }^{50,117}$, M. Punturo ${ }^{70}$, F. Puosi ${ }^{20,21}$, P. Puppo ${ }^{48}$, M. Pürrer ${ }^{101}$, H. Qi ${ }^{17}$, V. Quetschke ${ }^{143}$, P. J. Quinonez ${ }^{35}$, R. Quitzow-James ${ }^{82}$, F. J. Raab ${ }^{63}$, G. Raaijmakers ${ }^{50,248}$, H. Radkins ${ }^{63}$, N. Radulesco ${ }^{88}$, P. Raffai ${ }^{146}$, S. X. Rail ${ }^{222}$, S. Raja ${ }^{81}$, C. Rajan ${ }^{81}$, K. E. Ramirez ${ }^{143}$, T. D. Ramirez ${ }^{25}$, A. Ramos-Buades ${ }^{101}$, J. Rana ${ }^{141}$, P. Rapagnani ${ }^{48,93}$, U. D. Rapol ${ }^{257}$, B. Ratto ${ }^{35}$, V. Raymond ${ }^{17}$, N. Raza ${ }^{172}$, M. Razzano ${ }^{20,21}$, J. $\operatorname{Read}^{25}$, L. A. Rees ${ }^{183}$, T. Regimbau ${ }^{49}$, L. Rei ${ }^{80}$, S. Reid ${ }^{32}$, D. H. Reitze ${ }^{1,42}$, P. Relton ${ }^{17}$, P. Rettegno ${ }^{53,258}$, F. Ricci ${ }^{48,93}$, C. J. Richardson ${ }^{35}$, J. W. Richardson ${ }^{1}$, L. Richardson ${ }^{134}$, P. M. Ricker ${ }^{26}$, G. Riemenschneider ${ }^{53,258}$, K. Riles ${ }^{178}$, M. Rizzo ${ }^{15}$, N. A. Robertson ${ }^{1,66}$, R. Robie ${ }^{1}$, F. Robinet ${ }^{40}$, A. Rocchi ${ }^{15}$, J. A. Rocha ${ }^{25}$, S. Rodriguez ${ }^{25}$, R. D. Rodriguez-Soto ${ }^{35}$, L. Rolland $^{49}$, J. G. Rollins ${ }^{1}$, V. J. Roma ${ }^{56}$, M. Romanelli ${ }^{94}$, R. Romano ${ }^{4,5}$, C. L. Romel ${ }^{63}$, A. Romero ${ }^{207}$, I. M. Romero-Shaw ${ }^{6}$, J. H. Romie ${ }^{8}$, C. A. Rose ${ }^{29}$, D. Rosińska ${ }^{98}$, S. G. Rosofsky ${ }^{26}$, M. P. Ross ${ }^{230}$, S. Rowan ${ }^{66}$, S. J. Rowlinson ${ }^{14}$, Santosh Roy ${ }^{3}$, Soumen Roy $^{259}$, D. Rozza ${ }^{112,113}$, P. Ruggi ${ }^{41}$, K. Ryan ${ }^{63}$, S. Sachdev ${ }^{141}$, T. Sadecki ${ }^{63}$, J. Sadiq ${ }^{149}$, N. Sago ${ }^{260}$, S. Saito ${ }^{24}$, Y. Saito ${ }^{185}$, K. Sakai ${ }^{261}$, Y. Sakai ${ }^{189}$, M. Sakellariadou ${ }^{132}$, Y. Sakuno ${ }^{121}$, O. S. Salafia ${ }^{60,61,62}{ }^{\text {, L. Salconi }}{ }^{41}$, M. Saleem ${ }^{43}$, F. Salemi ${ }^{175,176}$, A. Samajdar ${ }^{50,117}$, E. J. Sanchez ${ }^{1}$, J. H. Sanchez ${ }^{25}$, L. E. Sanchez ${ }^{1}$, N. Sanchis-Gual ${ }^{262}$, J. R. Sanders ${ }^{263}$,

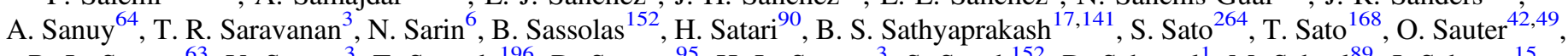
R. L. Savage ${ }^{63}$, V. Savant ${ }^{3}$, T. Sawada ${ }^{196}$, D. Sawant ${ }^{95}$, H. L. Sawant ${ }^{3}$, S. Sayah ${ }^{152}$, D. Schaetzl ${ }^{1}$, M. Scheel ${ }^{89}$, J. Scheuer $^{15}$, A. Schindler-Tyka ${ }^{42}$, P. Schmidt ${ }^{14}$, R. Schnabel ${ }^{148}$, M. Schneewind ${ }^{10,11}$, R. M. S. Schofield ${ }^{56}$, A. Schönbeck ${ }^{148}$, B. W. Schulte ${ }^{10,11}$, B. F. Schutz ${ }^{10,17}$, E. Schwartz ${ }^{17}$, J. Scott ${ }^{66}$, S. M. Scott ${ }^{9}$, M. Seglar-Arroyo ${ }^{49}$, E. Seidel ${ }^{26}$, T. Sekiguchi ${ }^{31}$, Y. Sekiguchi ${ }^{265}$, D. Sellers ${ }^{8}$, A. S. Sengupta ${ }^{259}$, N. Sennett ${ }^{101}$, D. Sentenac ${ }^{41}$, E. G. Seo ${ }^{104}$, V. Sequino ${ }^{5,27}$, A. Sergeev ${ }^{210}$, Y. Setyawati $^{10,11}$, T. Shaffer ${ }^{63}$, M. S. Shahriar ${ }^{15}$, B. Shams ${ }^{164}$, L. Shao ${ }^{193}$, S. Sharifi ${ }^{2}$, A. Sharma ${ }^{18,19}$, P. Sharma ${ }^{81}$, P. Shawhan ${ }^{100}$,

N. S. Shcheblanov ${ }^{226}$, H. Shen ${ }^{26}$, S. Shibagaki ${ }^{121}$, M. Shikauchi ${ }^{31}$, R. Shimizu ${ }^{24}$, T. Shimoda ${ }^{30}$, K. Shimode ${ }^{185}$, R. Shink ${ }^{222}$,

H. Shinkai ${ }^{266}$, T. Shishido ${ }^{47}$, A. Shoda ${ }^{23}$, D. H. Shoemaker ${ }^{67}$, D. M. Shoemaker ${ }^{224}$, K. Shukla ${ }^{187}$, S. ShyamSundar ${ }^{81}$,

M. Sieniawska ${ }^{98}$, D. Sigg $^{63}$, L. P. Singer ${ }^{107}$, D. Singh $^{141}$, N. $\operatorname{Singh}^{98}$, A. Singha ${ }^{50,147}$, A. M. Sintes ${ }^{137}$, V. Sipala ${ }^{112,113}$, V. Skliris ${ }^{17}$, B. J. J. Slagmolen, T. J. Slaven-Blair ${ }^{90}$, J. Smetana ${ }^{14}$, J. R. Smith ${ }^{25}$, R. J. E. Smith ${ }^{6}$, S. N. Somala ${ }^{267}$, K. Somiya ${ }^{208}$, E. J. Son ${ }^{52}$, K. Soni ${ }^{3}$, S. Soni ${ }^{2}$, B. Sorazu ${ }^{66}$, V. Sordini ${ }^{129}$, F. Sorrentino ${ }^{80}$, N. Sorrentino ${ }^{20,21}$, H. Sotani ${ }^{268}$, R. Soulard ${ }^{88}$, T. Souradeep ${ }^{3,257}$, E. Sowell ${ }^{140}$, V. Spagnuolo ${ }^{50,147}$, A. P. Spencer ${ }^{66}$, M. Spera ${ }^{72,73}$, A. K. Srivastava ${ }^{75}$, V. Srivastava ${ }^{57}$, K. Staats ${ }^{15}$, C. Stachie ${ }^{88}$, D. A. Steer ${ }^{36}$, J. Steinlechner ${ }^{50,147}$, S. Steinlechner ${ }^{50,147}$, D. J. Stops ${ }^{14}$, M. Stover ${ }^{165}$, K. A. Strain ${ }^{66}$, L. C. Strang ${ }^{111}$, G. Stratta ${ }^{87,269}$, A. Strunk ${ }^{63}$, R. Sturani ${ }^{251}$, A. L. Stuver ${ }^{103}$, J. Südbeck ${ }^{148}$, S. Sudhagar ${ }^{3}$, V. Sudhir ${ }^{67}$, R. Sugimoto ${ }^{198,270}$, H. G. Suh ${ }^{29}$, T. Z. Summerscales ${ }^{271}$, H. Sun ${ }^{90}$, L. Sun ${ }^{1,9}$, S. Sunil ${ }^{75}$, A. Sur ${ }^{76}$, J. Suresh ${ }^{31,37}$, P. J. Sutton ${ }^{17}$, Takamasa Suzuki ${ }^{168}$, 
Toshikazu Suzuki $^{37}$, B. L. Swinkels ${ }^{50}$, M. J. Szczepańczyk ${ }^{42}$, P. Szewczyk ${ }^{98}$, M. Tacca ${ }^{50}$, H. Tagoshi ${ }^{37}$, S. C. Tait ${ }^{66}$, H. Takahashi ${ }^{272}$, R. Takahashi ${ }^{23}$, A. Takamori ${ }^{39}$, S. Takano ${ }^{30}$, H. Takeda ${ }^{30}$, M. Takeda ${ }^{196}$, C. Talbot ${ }^{1}$, H. Tanaka ${ }^{273}$, Kazuyuki Tanaka ${ }^{196}$, Kenta Tanaka ${ }^{273}$, Taiki Tanaka ${ }^{37}$, Takahiro Tanaka ${ }^{260}$, A. J. Tanasijczuk ${ }^{97}$, S. Tanioka ${ }^{23,47}$, D. B. Tanner ${ }^{42}$, D. Tao ${ }^{1}$, A. Tapia ${ }^{25}$, E. N. Tapia San Martin ${ }^{23}$, E. N. Tapia San Martin ${ }^{50}$, J. D. Tasson ${ }^{186}$, S. Telada ${ }^{274}$, R. Tenorio ${ }^{137}$, L. Terkowski ${ }^{14}$, M. Test ${ }^{29}$, M. P. Thirugnanasambandam ${ }^{3}$, M. Thomas ${ }^{8}$, P. Thomas ${ }^{63}$, J. E. Thompson ${ }^{17}$, S. R. Thondapu ${ }^{81}$, K. A. Thorne ${ }^{8}$, E. Thrane ${ }^{6}$, Shubhanshu Tiwari ${ }^{155}$, Srishti Tiwari ${ }^{173}$, V. Tiwari ${ }^{17}$, K. Toland ${ }^{66}$, A. E. Tolley ${ }^{150}$, T. Tomaru ${ }^{23}$, Y. Tomigami ${ }^{196}$, T. Tomura ${ }^{185}$, M. Tonelli ${ }^{20,21}$, A. Torres-Forné ${ }^{118}$, C. I. Torrie ${ }^{1}$, I. Tosta e Melo ${ }^{112,113}$, D. Töyrä9 ${ }^{2}$

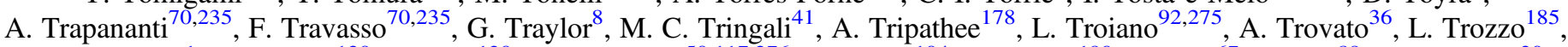
R. J. Trudeau ${ }^{1}$, D. S. Tsai ${ }^{120}$, D. Tsai ${ }^{120}$, K. W. Tsang ${ }^{50,117,276}$, T. Tsang ${ }^{104}$, J-S. Tsao ${ }^{190}$, M. Tse ${ }^{67}$, R. Tso ${ }^{89}$, K. Tsubono ${ }^{30}$, S. Tsuchida ${ }^{196}$, L. Tsukada ${ }^{31}$, D. Tsuna ${ }^{31}$, T. Tsutsui ${ }^{31}$, T. Tsuzuki ${ }^{24}$, M. Turconi ${ }^{88}$, D. Tuyenbayev ${ }^{128}$, A. S. Ubhi ${ }^{14}$, N. Uchikata $^{37}$, T. Uchiyama ${ }^{185}$, R. P. Udall ${ }^{1,102}$, A. Ueda ${ }^{180}$, T. Uehara ${ }^{277,278}$, K. Ueno ${ }^{31}$, G. Ueshima ${ }^{279}$, D. Ugolini ${ }^{280}$, C. S. Unnikrishnan ${ }^{173}$, F. Uraguchi ${ }^{24}$, A. L. Urban ${ }^{2}$, T. Ushiba ${ }^{185}$, S. A. Usman ${ }^{125}$, A. C. Utina ${ }^{50,147}$, H. Vahlbruch ${ }^{10,11}$, G. Vajente ${ }^{1}$, A. Vajpeyi ${ }^{6}$, G. Valdes ${ }^{2}$, M. Valentini ${ }^{175,176}$, V. Valsan ${ }^{29}$, N. van Bakel $^{50}$, M. van Beuzekom ${ }^{50}$, J. F. J. van den Brand ${ }^{50,99,147}$, C. Van Den Broeck ${ }^{50,117}$, D. C. Vander-Hyde ${ }^{57}$, L. van der Schaaf ${ }^{50}$, J. V. van Heijningen ${ }^{90,97}$, J. Vanosky ${ }^{1}$,

M. H. P. M. van Putten ${ }^{281}$, M. Vardaro ${ }^{50,228}$, A. F. Vargas ${ }^{111}$, V. Varma ${ }^{89}$, M. Vasúth ${ }^{68}$, A. Vecchio ${ }^{14}$, G. Vedovato ${ }^{73}$, J. Veitch $^{66}$, P. J. Veitch ${ }^{78}$, K. Venkateswara ${ }^{230}$, J. Venneberg ${ }^{10,11}$, G. Venugopalan ${ }^{1}$, D. Verkindt ${ }^{49}$, Y. Verma ${ }^{81}$, D. Veske ${ }^{45}$, F. Vetrano $^{86}$, A. Vicere ${ }^{86,87}$, A. D. Viets ${ }^{234}$, V. Villa-Ortega ${ }^{149}$, J.-Y. Vinet ${ }^{88}$, S. Vitale ${ }^{67}$, T. Vo ${ }^{57}$, H. Vocca ${ }^{70,71}$, E. R. G. von Reis ${ }^{63}$, J. von Wrangel ${ }^{10,11}$, C. Vorvick ${ }^{63}$, S. P. Vyatchanin ${ }^{83}$, L. E. Wade ${ }^{165}$, M. Wade ${ }^{165}$, K. J. Wagner ${ }^{119}$, R. C. Walet ${ }^{50}$, M. Walker ${ }^{7}$, G. S. Wallace ${ }^{32}$, L. Wallace ${ }^{1}$, S. Walsh ${ }^{29}$, J. Wang ${ }^{169}$, J. Z. Wang ${ }^{178}$, W. H. Wang ${ }^{143}$, R. L. Ward ${ }^{9}$, J. Warner ${ }^{63}$, M. Was ${ }^{49}$,

T. Washimi ${ }^{23}$, N. Y. Washington ${ }^{1}$, J. Watchi ${ }^{138}$, B. Weaver ${ }^{63}$, L. Wei $^{10,11}$, M. Weinert ${ }^{10,11}$, A. J. Weinstein ${ }^{1}$, R. Weiss ${ }^{67}$, C. M. Weller ${ }^{230}$, F. Wellmann ${ }^{10,11}$, L. Wen ${ }^{90}$, P. Weßels ${ }^{10,11}$, J. W. Westhouse ${ }^{35}$, K. Wette ${ }^{9}$, J. T. Whelan ${ }^{119}$, D. D. White ${ }^{25}$, B. F. Whiting ${ }^{42}$, C. Whittle ${ }^{67}$, D. Wilken ${ }^{10,11}$, D. Williams ${ }^{66}$, M. J. Williams ${ }^{66}$, A. R. Williamson ${ }^{150}$, J. L. Willis ${ }^{1}$, B. Willke ${ }^{10,11}$, D. J. Wilson ${ }^{134}$, W. Winkler ${ }^{10,11}$, C. C. Wipf ${ }^{1}$, T. Wlodarczyk ${ }^{101}$, G. Woan ${ }^{66}$, J. Woehler ${ }^{10,11}$, J. K. Wofford ${ }^{119}$, I. C. F. Wong ${ }^{104}$, C. $\mathrm{Wu}^{124}$, D. S. $\mathrm{Wu}^{10,11}$, H. Wu ${ }^{124}$, S. Wu ${ }^{124}$, D. M. Wysocki ${ }^{29,119}$, L. Xiao ${ }^{1}$, W-R. Xu ${ }^{190}$, T. Yamada ${ }^{273}$, H. Yamamoto $^{1}$, Kazuhiro Yamamoto ${ }^{184}$, Kohei Yamamoto ${ }^{273}$, T. Yamamoto ${ }^{185}$, K. Yamashita ${ }^{184}$, R. Yamazaki ${ }^{192}$, F. W. Yang ${ }^{164}$, L. Yang ${ }^{159}$,

Yang Yang ${ }^{42}$, Yi Yang ${ }^{282}$, Z. Yang ${ }^{59}$, M. J. Yap ${ }^{9}$, D. W. Yeeles ${ }^{17}$, A. B. Yelikar ${ }^{119}$, M. Ying ${ }^{120}$, K. Yokogawa ${ }^{195}$,

J. Yokoyama $^{30,31}$, T. Yokozawa ${ }^{185}$, A. Yoon ${ }^{7}$, T. Yoshioka ${ }^{195}$, Hang Yu ${ }^{89}$, Haocun Yu ${ }^{67}$, H. Yuzurihara ${ }^{37}$, A. Zadrożny ${ }^{219}$, M. Zanolin ${ }^{35}$, S. Zeidler ${ }^{283}$, T. Zelenova ${ }^{41}$, J.-P. Zendri ${ }^{73}$, M. Zevin ${ }^{15}$, M. Zhan ${ }^{169}$, H. Zhang ${ }^{190}$, J. Zhang ${ }^{90}$, L. Zhang ${ }^{1}$, R. Zhang ${ }^{42}$, T. Zhang ${ }^{14}$, C. Zhao ${ }^{90}$, G. Zhao ${ }^{138}$, Yue Zhao ${ }^{164}$, Yuhang Zhao ${ }^{23}$, Z. Zhou ${ }^{15}$, X. J. Zhu ${ }^{6}$, Z.-H. Zhu ${ }^{110}$, M. E. Zucker ${ }^{1,67}$, and J. Zweizig ${ }^{1}$

${ }^{1}$ LIGO Laboratory, California Institute of Technology, Pasadena, CA 91125, USA

${ }^{2}$ Louisiana State University, Baton Rouge, LA 70803, USA

${ }^{3}$ Inter-University Centre for Astronomy and Astrophysics, Pune 411007, India

${ }^{4}$ Dipartimento di Farmacia, Università di Salerno, I-84084 Fisciano, Salerno, Italy

${ }^{5}$ INFN, Sezione di Napoli, Complesso Universitario di Monte S.Angelo, I-80126 Napoli, Italy

${ }^{6}$ OzGrav, School of Physics \& Astronomy, Monash University, Clayton 3800, Victoria, Australia

Christopher Newport University, Newport News, VA 23606, USA

${ }^{8}$ LIGO Livingston Observatory, Livingston, LA 70754, USA

${ }^{9}$ OzGrav, Australian National University, Canberra, Australian Capital Territory 0200, Australia

${ }^{10}$ Max Planck Institute for Gravitational Physics (Albert Einstein Institute), D-30167 Hannover, Germany

${ }^{11}$ Leibniz Universität Hannover, D-30167 Hannover, Germany

${ }^{12}$ University of Cambridge, Cambridge CB2 1TN, UK

${ }^{13}$ Theoretisch-Physikalisches Institut, Friedrich-Schiller-Universität Jena, D-07743 Jena, Germany

${ }^{14}$ University of Birmingham, Birmingham B15 2TT, UK

${ }^{15}$ Center for Interdisciplinary Exploration \& Research in Astrophysics (CIERA), Northwestern University, Evanston, IL 60208, USA

${ }^{16}$ Instituto Nacional de Pesquisas Espaciais, 12227-010 São José dos Campos, São Paulo, Brazil

${ }^{17}$ Gravity Exploration Institute, Cardiff University, Cardiff CF24 3AA, UK

${ }^{18}$ Gran Sasso Science Institute (GSSI), I-67100 L'Aquila, Italy

${ }^{19}$ INFN, Laboratori Nazionali del Gran Sasso, I-67100 Assergi, Italy ${ }^{20}$ INFN, Sezione di Pisa, I-56127 Pisa, Italy

${ }^{21}$ Università di Pisa, I-56127 Pisa, Italy

${ }^{22}$ International Centre for Theoretical Sciences, Tata Institute of Fundamental Research, Bengaluru 560089, India

${ }^{23}$ Gravitational Wave Science Project, National Astronomical Observatory of Japan (NAOJ), Mitaka City, Tokyo 181-8588, Japan

${ }^{24}$ Advanced Technology Center, National Astronomical Observatory of Japan (NAOJ), Mitaka City, Tokyo 181-8588, Japan

${ }^{25}$ California State University Fullerton, Fullerton, CA 92831, USA

${ }^{26}$ NCSA, University of Illinois at Urbana-Champaign, Urbana, IL 61801, USA

${ }^{27}$ Università di Napoli "Federico II," Complesso Universitario di Monte S.Angelo, I-80126 Napoli, Italy

${ }^{28}$ Université de Lyon, Université Claude Bernard Lyon 1, CNRS, Institut Lumière Matière, F-69622 Villeurbanne, France

${ }^{29}$ University of Wisconsin-Milwaukee, Milwaukee, WI 53201, USA

${ }^{30}$ Department of Physics, The University of Tokyo, Bunkyo-ku, Tokyo 113-0033, Japan

${ }^{31}$ Research Center for the Early Universe (RESCEU), The University of Tokyo, Bunkyo-ku, Tokyo 113-0033, Japan

${ }^{32}$ SUPA, University of Strathclyde, Glasgow G1 1XQ, UK

${ }^{33}$ Dipartimento di Matematica e Informatica, Università di Udine, I-33100 Udine, Italy

${ }^{34}$ INFN, Sezione di Trieste, I-34127 Trieste, Italy

${ }^{35}$ Embry-Riddle Aeronautical University, Prescott, AZ 86301, USA

${ }^{36}$ Université de Paris, CNRS, Astroparticule et Cosmologie, F-75006 Paris, France

${ }^{37}$ Institute for Cosmic Ray Research (ICRR), KAGRA Observatory, The University of Tokyo, Kashiwa City, Chiba 277-8582, Japan 
${ }^{38}$ Accelerator Laboratory, High Energy Accelerator Research Organization (KEK), Tsukuba City, Ibaraki 305-0801, Japan

${ }^{39}$ Earthquake Research Institute, The University of Tokyo, Bunkyo-ku, Tokyo 113-0032, Japan

${ }^{40}$ Université Paris-Saclay, CNRS/IN2P3, IJCLab, F-91405 Orsay, France

${ }^{41}$ European Gravitational Observatory (EGO), I-56021 Cascina, Pisa, Italy

${ }^{43}$ University of Florida, Gainesville, FL 32611, USA
${ }^{2}$ Chennai Mathematical Institute, Chennai 603103, India

${ }^{44}$ Department of Mathematics and Physics, Hirosaki University, Hirosaki City, Aomori 036-8561, Japan

${ }^{45}$ Columbia University, New York, NY 10027, USA

${ }^{46}$ Kamioka Branch, National Astronomical Observatory of Japan (NAOJ), Kamioka-cho, Hida City, Gifu 506-1205, Japan

${ }^{47}$ The Graduate University for Advanced Studies (SOKENDAI), Mitaka City, Tokyo 181-8588, Japan

${ }^{48}$ INFN, Sezione di Roma, I-00185 Roma, Italy

${ }^{49}$ Univ. Grenoble Alpes, Laboratoire d'Annecy de Physique des Particules (LAPP), Université Savoie Mont Blanc, CNRS/IN2P3, F-74941 Annecy, France 50 Nikhef, Science Park 105, 1098 XG Amsterdam, The Netherlands

${ }^{51}$ Korea Institute of Science and Technology Information (KISTI), Yuseong-gu, Daejeon 34141, Republic of Korea

${ }^{52}$ National Institute for Mathematical Sciences, Daejeon 34047, Republic of Korea

53 INFN Sezione di Torino, I-10125 Torino, Italy
${ }^{54}$ International College, Osaka University, Toyonaka City, Osaka 560-0043, Japan

${ }^{55}$ School of High Energy Accelerator Science, The Graduate University for Advanced Studies (SOKENDAI), Tsukuba City, Ibaraki 305-0801, Japan

${ }_{57}^{56}$ University of Oregon, Eugene, OR 97403, USA

${ }_{58}^{57}$ Syracuse University, Syracuse, NY 13244, USA

${ }^{58}$ Université de Liège, B-4000 Liège, Belgium

${ }^{59}$ University of Minnesota, Minneapolis, MN 55455, USA

${ }^{60}$ Università degli Studi di Milano-Bicocca, I-20126 Milano, Italy

${ }^{61}$ INFN, Sezione di Milano-Bicocca, I-20126 Milano, Italy

${ }^{62}$ INAF, Osservatorio Astronomico di Brera sede di Merate, I-23807 Merate, Lecco, Italy

${ }^{63}$ LIGO Hanford Observatory, Richland, WA 99352, USA

${ }^{64}$ Institut de Ciències del Cosmos, Universitat de Barcelona, C/ Martí i Franquès 1, Barcelona, E-08028, Spain

${ }^{65}$ Dipartimento di Medicina, Chirurgia e Odontoiatria "Scuola Medica Salernitana," Università di Salerno, I-84081 Baronissi, Salerno, Italy

${ }^{66}$ SUPA, University of Glasgow, Glasgow G12 8QQ, UK

${ }^{67}$ LIGO Laboratory, Massachusetts Institute of Technology, Cambridge, MA 02139, USA

${ }^{68}$ Wigner RCP, RMKI, H-1121 Budapest, Konkoly Thege Miklós út 29-33, Hungary

${ }^{69}$ Stanford University, Stanford, CA 94305, USA

${ }^{70}$ INFN, Sezione di Perugia, I-06123 Perugia, Italy

${ }^{71}$ Università di Perugia, I-06123 Perugia, Italy

${ }^{72}$ Università di Padova, Dipartimento di Fisica e Astronomia, I-35131 Padova, Italy

${ }^{73}$ INFN, Sezione di Padova, I-35131 Padova, Italy

${ }^{74}$ Montana State University, Bozeman, MT 59717, USA

${ }^{75}$ Institute for Plasma Research, Bhat, Gandhinagar 382428, India

${ }^{76}$ Nicolaus Copernicus Astronomical Center, Polish Academy of Sciences, 00-716, Warsaw, Poland

${ }^{77}$ Dipartimento di Ingegneria, Università del Sannio, I-82100 Benevento, Italy

${ }^{78}$ OzGrav, University of Adelaide, Adelaide, South Australia 5005, Australia

${ }^{79}$ California State University, Los Angeles, 5151 State University Dr., Los Angeles, CA 90032, USA

80 INFN, Sezione di Genova, I-16146 Genova, Italy

81 RRCAT, Indore, Madhya Pradesh 452013, India
${ }^{82}$ Missouri University of Science and Technology, Rolla, MO 65409, USA

${ }^{83}$ Faculty of Physics, Lomonosov Moscow State University, Moscow 119991, Russia

${ }^{84}$ SUPA, University of the West of Scotland, Paisley PA1 2BE, UK

${ }^{85}$ Bar-Ilan University, Ramat Gan, 5290002, Israel

${ }_{87}^{86}$ Università degli Studi di Urbino "Carlo Bo," I-61029 Urbino, Italy

${ }^{87}$ INFN, Sezione di Firenze, I-50019 Sesto Fiorentino, Firenze, Italy

${ }^{88}$ Artemis, Université Côte d'Azur, Observatoire de la Côte d'Azur, CNRS, F-06304 Nice, France

${ }^{89}$ CaRT, California Institute of Technology, Pasadena, CA 91125, USA

${ }^{90}$ OzGrav, University of Western Australia, Crawley, Western Australia 6009, Australia

${ }^{91}$ Dipartimento di Fisica "E.R. Caianiello," Università di Salerno, I-84084 Fisciano, Salerno, Italy

92 INFN, Sezione di Napoli, Gruppo Collegato di Salerno, Complesso Universitario di Monte S. Angelo, I-80126 Napoli, Italy

${ }^{93}$ Università di Roma "La Sapienza," I-00185 Roma, Italy

${ }^{94}$ Univ Rennes, CNRS, Institut FOTON - UMR6082, F-3500 Rennes, France

95 Indian Institute of Technology Bombay, Powai, Mumbai 400 076, India

${ }^{96}$ Laboratoire Kastler Brossel, Sorbonne Université, CNRS, ENS-Université PSL, Collège de France, F-75005 Paris, France

${ }_{98}^{97}$ Université catholique de Louvain, B-1348 Louvain-la-Neuve, Belgium

98 Astronomical Observatory Warsaw University, 00-478 Warsaw, Poland

${ }^{99}$ VU University Amsterdam, 1081 HV Amsterdam, The Netherlands

${ }^{100}$ University of Maryland, College Park, MD 20742, USA

${ }^{101}$ Max Planck Institute for Gravitational Physics (Albert Einstein Institute), D-14476 Potsdam, Germany

${ }^{102}$ School of Physics, Georgia Institute of Technology, Atlanta, GA 30332, USA

${ }_{103}$ Villanova University, 800 Lancaster Ave., Villanova, PA 19085, USA

${ }^{104}$ Faculty of Science, Department of Physics, The Chinese University of Hong Kong, Shatin, N.T., Hong Kong

${ }^{105}$ Stony Brook University, Stony Brook, NY 11794, USA

${ }^{106}$ Center for Computational Astrophysics, Flatiron Institute, New York, NY 10010, USA

${ }^{107}$ NASA Goddard Space Flight Center, Greenbelt, MD 20771, USA

${ }^{108}$ Dipartimento di Fisica, Università degli Studi di Genova, I-16146 Genova, Italy

${ }^{109}$ Tsinghua University, Beijing 100084, People's Republic of China

${ }^{110}$ Department of Astronomy, Beijing Normal University, Beijing 100875, People's Republic of China

${ }^{111}$ OzGrav, University of Melbourne, Parkville, Victoria 3010, Australia

112 Università degli Studi di Sassari, I-07100 Sassari, Italy

${ }^{113}$ INFN, Laboratori Nazionali del Sud, I-95125 Catania, Italy 


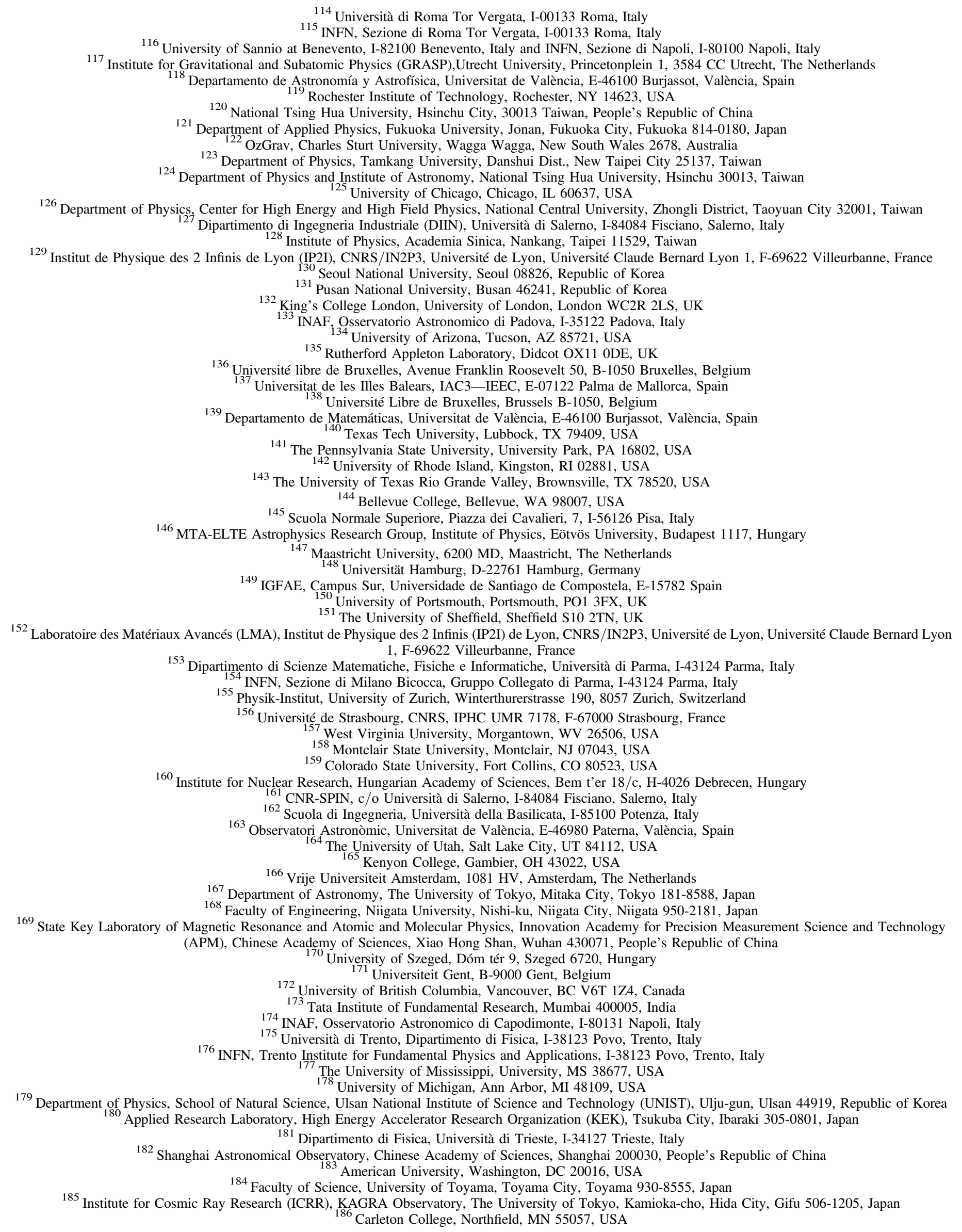




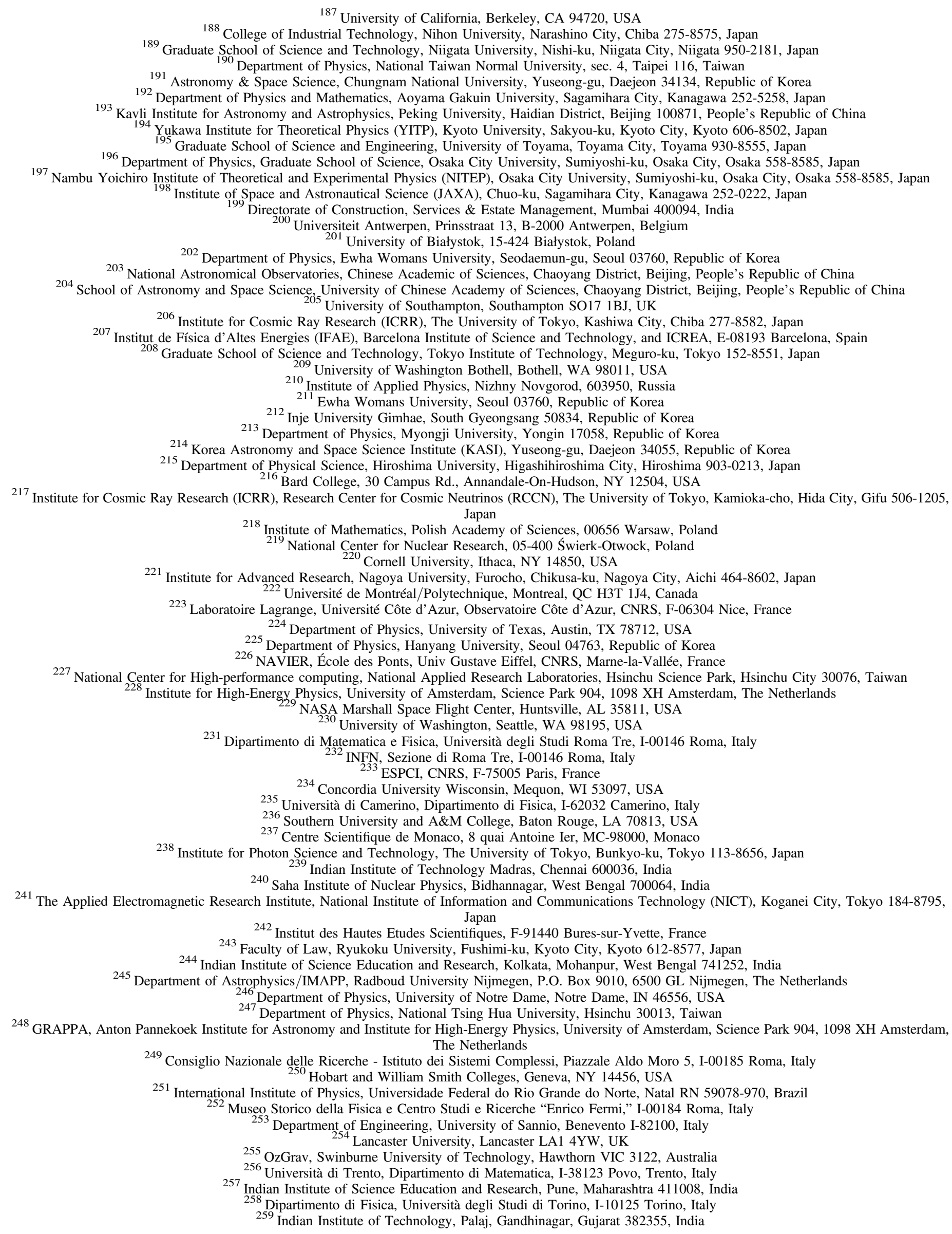




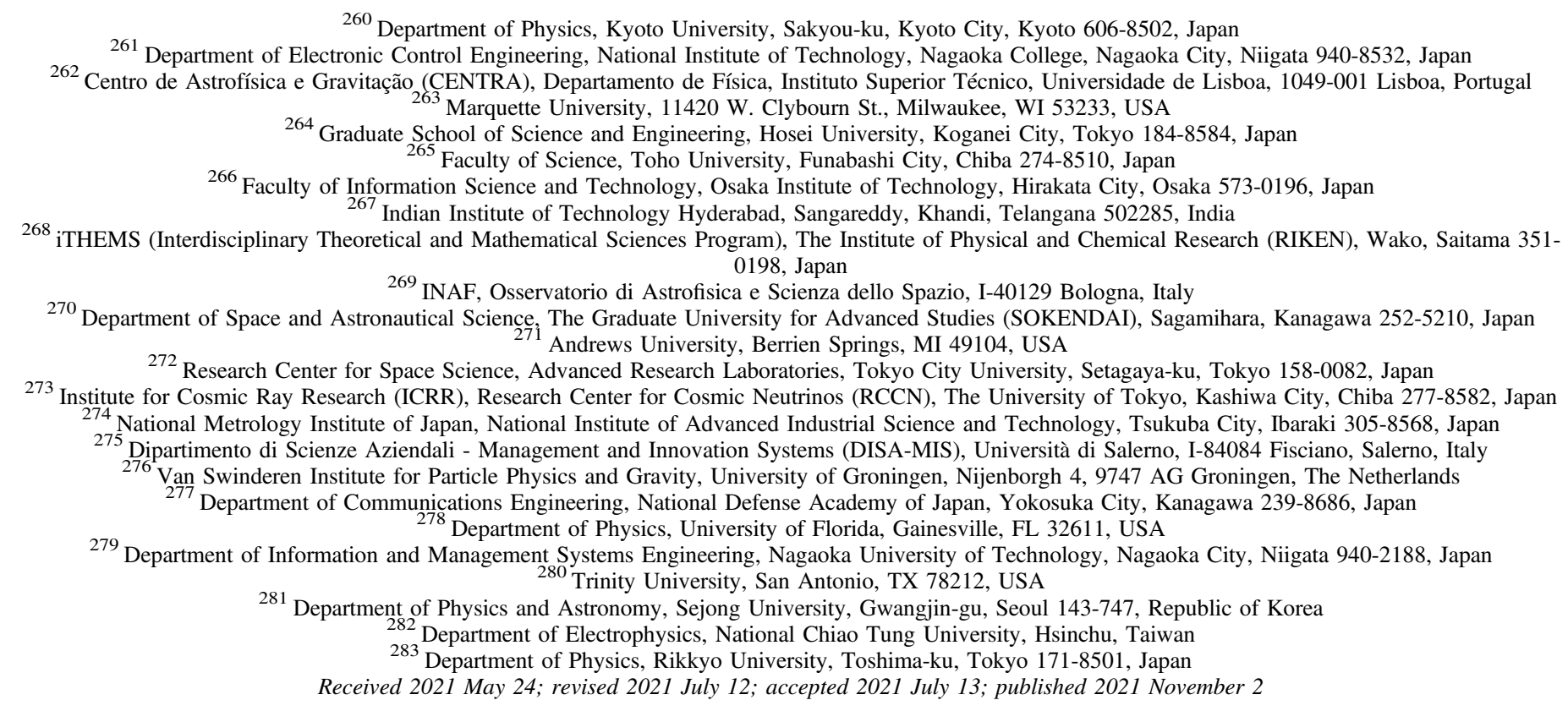

\begin{abstract}
We present results of three wide-band directed searches for continuous gravitational waves from 15 young supernova remnants in the first half of the third Advanced LIGO and Virgo observing run. We use three search pipelines with distinct signal models and methods of identifying noise artifacts. Without ephemerides of these sources, the searches are conducted over a fRequency band spanning from 10 to $2 \mathrm{kHz}$. We find no evidence of continuous gravitational radiation from these sources. We set upper limits on the intrinsic signal strain at $95 \%$ confidence level in sample subbands, estimate the sensitivity in the full band, and derive the corresponding constraints on the fiducial neutron star ellipticity and $r$-mode amplitude. The best $95 \%$ confidence constraints placed on the signal strain are $7.7 \times 10^{-26}$ and $7.8 \times 10^{-26}$ near $200 \mathrm{~Hz}$ for the supernova remnants G39.2-0.3 and G65.7 +1.2 , respectively. The most stringent constraints on the ellipticity and $r$-mode amplitude reach $\lesssim 10^{-7}$ and $\lesssim 10^{-5}$, respectively, at frequencies above $\sim 400 \mathrm{~Hz}$ for the closest supernova remnant G266.2-1.2/Vela Jr.
\end{abstract}

Unified Astronomy Thesaurus concepts: Gravitational waves (678); Gravitational wave astronomy (675); Supernova remnants (1667); Neutron stars (1108)

\section{Introduction}

Transient gravitational waves (GWs) from compact binary coalescences (Abbott et al. 2019b, 2021a) have been directly observed by the Advanced Laser Interferometer GravitationalWave Observatory (Advanced LIGO) detectors (Aasi et al. 2015a) and the Advanced Virgo detector (Acernese et al. 2015). Continuous GWs (CWs) have not yet been detected. The most likely sources of CWs detectable by ground-based interferometers are nonaxisymmetric, rapidly rotating neutron stars. Searches for CWs have been carried out targeting various isolated sources, including known pulsars with electromagnetic ephemerides (Abbott et al. 2019c, 2021b), neutron stars without ephemerides in the galactic center or in globular clusters (Aasi et al. 2013; Abbott et al. 2017; Dergachev et al. 2019; Piccinni et al. 2020), neutron stars in binary systems (Abbott et al. 2019d; Middleton et al. 2020; Zhang et al. 2021), and young supernova remnants (SNRs; Aasi et al. 2015b; Sun et al. 2016; Ming et al. 2019; Abbott et al. 2019e; Lindblom \& Owen 2020; Millhouse et al. 2020; Papa et al. 2020; Beniwal et al. 2021). Searches have also been conducted over the whole sky for CWs instead of targeting at a particular direction (Abbott et al. 2019f; Covas \& Sintes 2020; Dergachev \& Papa 2020; Abbott et al. 2021c; Steltner et al. 2021;

${ }^{284}$ Deceased, August 2020.
Wette et al. 2021). This work searches for CWs from SNRs in the first half of the third observing run $(\mathrm{O} 3 \mathrm{a})$, which commenced on 2019 April 1 and ended on 2020 March 27 (Acernese et al. 2015; Buikema et al. 2020).

Young neutron stars in SNRs are one potential source of continuous, quasi-monochromatic GWs. If pulsations are observed in electromagnetic emission from the neutron star, one can search for CWs guided by the ephemerides obtained from those observations, as in, e.g., Abbott et al. (2019c) and Abbott et al. (2020). Even so, there is no guarantee that the GW-emitting quadrupole is phase locked to the electromagnetic pulsations. When there is no phase locking, search algorithms are needed that can track small (and possibly randomly varying) displacements between the gravitational and electromagnetic frequencies (Abbott et al. 2019a; Beniwal et al. 2021). If the neutron star does not pulsate, it may be observed as an X-ray point source, known as a central compact object (Gotthelf et al. 2013). In the latter scenario, the maximum GW strain can be inferred from the age of the SNR (Wette et al. 2008; Riles 2013), as has been done in recent GW searches (Millhouse et al. 2020; Beniwal et al. 2021).

A rotating, nonaxisymmetric neutron star has a time-varying mass quadrupole (from the point of view of a distant observer) and emits GWs at a strain proportional to the stellar ellipticity, 
which is affected by the nuclear equation of state, the history of strain buildup and diffusion in the crust, and the magnetic field configuration (Glampedakis \& Gualtieri 2018). For an isolated star, young neutron stars may have larger nonaxisymmetries than older ones and consequently may produce stronger GW emissions (Knispel \& Allen 2008; Riles 2017). As the star ages, ohmic (Haensel et al. 1990), thermal (Gnedin et al. 2001; Potekhin et al. 2015), tectonic, or other relaxation processes work to reduce the asymmetries introduced in the birth process. Young neutron stars are therefore promising targets for $\mathrm{CW}$ searches. The GW frequency is proportional to the stellar spin frequency $f_{\star}$. For thermoelastic (Ushomirsky et al. 2000; Johnson-McDaniel \& Owen 2013) or magnetic (Cutler 2002; Mastrano et al. 2011; Lasky \& Melatos 2013) mass quadrupoles, the predicted frequency is either $f_{\star}$ or $2 f_{\star} ; r$-mode current quadrupoles emit at $\sim 4 f_{\star} / 3$ (Andersson 1998; Owen et al. 1998; Caride et al. 2019), with minor equation-of-statedependent corrections; also, pinned superfluids in neutron stars may produce CWs at frequencies proportional to $f_{\star}$ (Jones 2010; Melatos et al. 2015).

In young, rapidly rotating neutron stars, $f_{\star}$ evolves quickly under the action of gravitational and electromagnetic torques (Knispel \& Allen 2008; Riles 2013). Rapid spin-down in young SNRs creates challenges for traditional CW search methods, especially over a long observation with duration $T_{\text {obs }} \gtrsim 1 \mathrm{yr}$. Most previous searches for SNRs have been restricted to short ( $\sim 1$ month) stretches of data (e.g., Abadie et al. 2010; Abbott et al. 2019e), limited parameter space (e.g., Lindblom \& Owen 2020), or have had a high associated computational cost (e.g., Sun et al. 2016; Papa et al. 2020). Accounting for spindown in a coherent search requires a very large number of templates, which increases computation cost beyond feasibility. Furthermore, $f_{\star}$ may wander randomly, a phenomenon known as spin wandering or timing noise (Hobbs et al. 2010; Shannon $\&$ Cordes 2010; Price et al. 2012; Ashton et al. 2015; Namkham et al. 2019; Parthasarathy et al. 2019; Lower et al. 2020), due to unknown internal or magnetospheric processes (Cordes \& Greenstein 1981; Melatos \& Link 2014). One computationally efficient alternative to a coherent search is a semicoherent search in which the integration is calculated coherently on blocks of short duration $T_{\text {coh }}$ and added incoherently over the full $T_{\text {obs }}$.

We apply three semicoherent methods to search for signals from 15 known young SNRs in the data collected in the first half (6 months) of O3: the directed Band-Sampled-Data (BSD) pipeline (Piccinni et al. 2018), based on the FrequencyHough (FH) transform (Astone et al. 2014b; Antonucci et al. 2008), and the single-harmonic Viterbi and dual-harmonic Viterbi pipelines, both based on a hidden Markov model (HMM) tracking scheme (Sun et al. 2018, 2019). The two Viterbi methods achieve a lower sensitivity compared to the BSD pipeline but take into consideration the uncertainties associated with the star's stochastic spin evolution, with one of them tracking two harmonics of the star's spin frequency simultaneously (Sun et al. 2018, 2019), making the three methods complementary to each other.

The structure of the paper is as follows. In Section 2, we introduce the 15 young SNR targets, listing their location, estimated age, and distance. In Section 3, we briefly describe the interferometric data analyzed. In Section 4, we review each of the three search methods and the parameter space covered. The strain upper limits, estimated sensitivity, and astrophysical interpretation are discussed in Section 5. A conclusion is given in Section 6. The postprocessing procedure applied to the candidates identified in each search is presented in Appendix A. Technical details on the pipelines are described in Appendix B.

\section{Targeted Sources}

The target SNRs are selected from the Green supernova catalog (Green 2019) and the SNRcat, an online catalog of high-energy galactic SNRs hosted by the University of Manitoba (Ferrand \& Safi-Harb 2012; SNR 2020), as SNRs with X-ray point sources are likely to contain neutron stars. Of the 15 SNRs in Table 1, 7 are searched using all three different pipelines, while the remaining 8 are only searched by the single-harmonic Viterbi pipeline. The characteristic ages of the neutron stars are inferred from the estimated supernova ages listed in the table. In the three pipelines, we cover parts of different parameter spaces, corresponding to slightly different assumptions of the characteristic age of the star. See Section 4 for details for each pipeline.

The 15 SNRs were previously searched in the earlier LIGO observing runs, but no CW signal was identified (Abbott et al. 2019e; Lindblom \& Owen 2020; Millhouse et al. 2020; Papa et al. 2020). Additionally, Papa et al. (2020) performed a follow-up search for subthreshold candidates obtained in the first observing run of Advanced LIGO (O1) (Ming et al. 2019) for three of the SNRs, Cassiopeia A (Cas A), Vela Jr., and G347.3-0.5, using data collected in the second observing run of Advanced LIGO (O2), and reported one possible CW candidate in G347.3-0.5. This fully coherent follow-up search uses two stretches of data in $\mathrm{O} 2$ ( $T_{\text {coh }} \sim 4$ months each). As indicated in Table 1, only the single-harmonic Viterbi pipeline (which allows for stochastic spin wandering) searches G347.3-0.5 semicoherently using a short $T_{\text {coh }}$. Since the signal-to-noise ratio roughly scales $\propto T_{\text {coh }}^{1 / 2}$, the sensitivity presented in Papa et al. (2020) exceeds that presented here for G347.3-0.5, provided that the signal power leaked into adjacent frequency bins due to the spin-down and spin wandering over the coherent duration is negligible. In addition, the candidate reported in Papa et al. (2020) was originally identified as a subthreshold one. Therefore, it is not surprising that we do not find a possible candidate in G347.3-0.5.

\section{Instrumental Overview and Data}

The O3 observing run started on 2019 April 1 at 15:00 UTC and ended on 2020 March 27 at 17:00 UTC. For the search, we use data collected by the two Advanced LIGO detectors in Hanford, Washington (H), and Livingston, Louisiana (L), and Advanced Virgo in the first half of O3, from the start until 2019 October 1. This time period is referred to as "O3a." The data collected by the two LIGO detectors during the second half of O3 (O3b), starting from 2019 November 1 until the end of O3, are used by the BSD pipeline (Section 4.1) and dual-harmonic Viterbi pipeline (Section 4.3) to cross-check candidates. Data collected by Virgo are only used by the BSD pipeline, which runs the initial search using individual detectors separately (Section 4.1). In the two Viterbi-based pipelines, the Virgo data are not used owing to the detector's relatively lower sensitivity, and the two pipelines both operate on all detectors combined. All three pipelines use data collected when the detectors are in the nominal low-noise observing mode (Davis et al. 2021). The 
Table 1

The 15 SNRs Covered in This Analysis

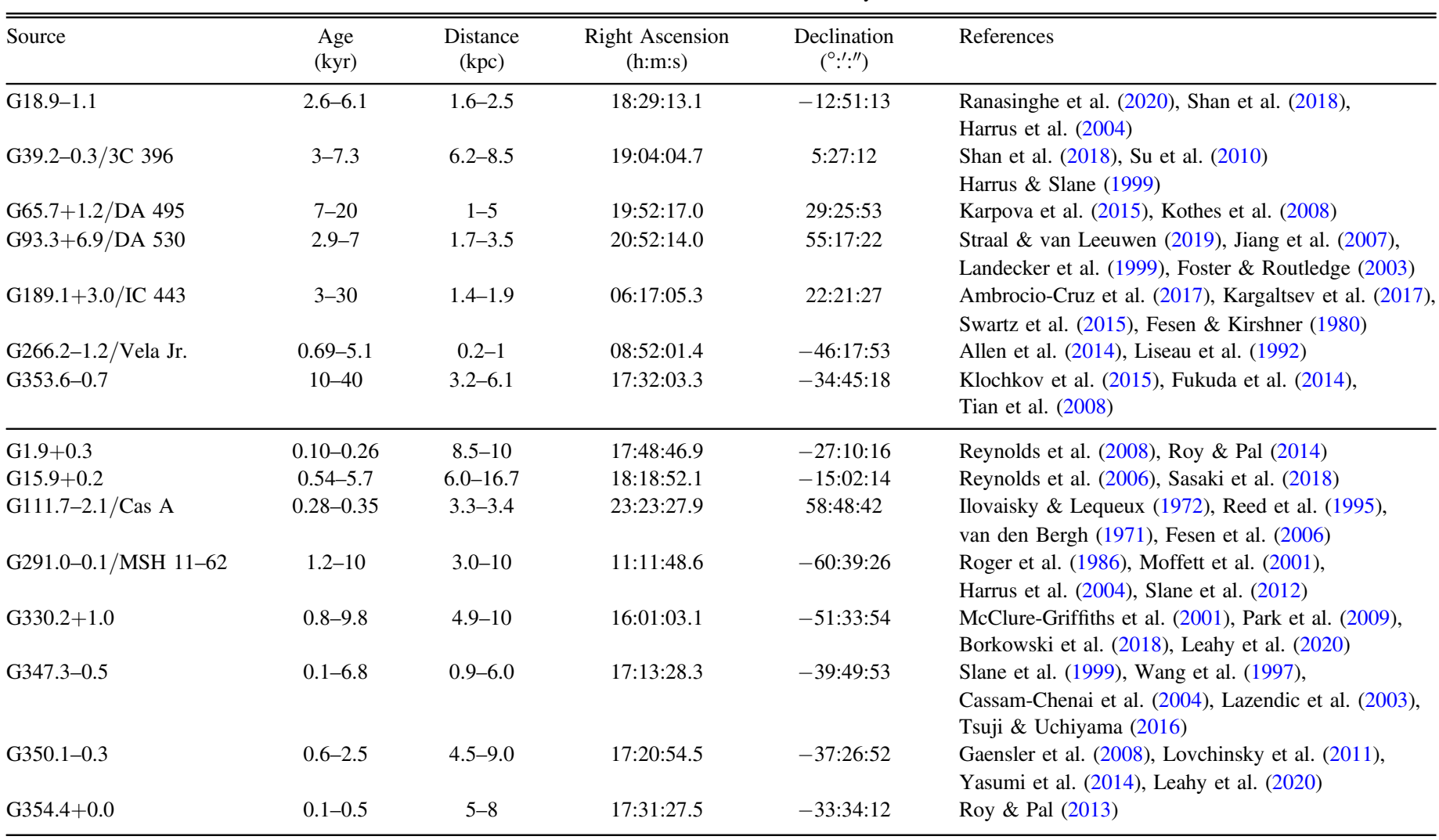

Note. Sources in the upper half of the table are searched by all three pipelines described in Section 4. Sources in the bottom half are searched by a single pipeline described in Section 4.2. The ages and distances listed are consistent with the values used in the previous LIGO analysis (Abbott et al. 2019e).

BSD pipeline (Section 4.1) uses low-latency calibrated data (C00 frames; Sun et al. 2020) for $\mathrm{H}$ and L detectors and the "online" calibration version for Virgo, after a procedure of removing significant short-duration noise transients, known as "glitches" (Davis et al. 2021), in the Short Fourier Transform Database (SFDB; Astone et al. 2005). Tests show that the difference between the C00 data, after glitch removal in SFDB, and glitch-gated $\mathrm{C} 01$ frames is negligible. The two Viterbi pipelines (Sections 4.2 and 4.3) use the high-latency calibrated data (C01 frames; Sun et al. 2020), passed through a procedure of glitch gating (Zweizig \& Riles 2020).

\section{Search Methods}

\subsection{BSD}

The BSD-directed search pipeline is a hierarchical semicoherent method based on the FH transform (Antonucci et al. 2008; Astone et al. 2014b). A previous search using the BSDdirected search pipeline, pointing to the Galactic center in Advanced LIGO O2, was reported in Piccinni et al. (2020). The pipeline descibed in this section is based on the BSD framework, i.e., a library of functions that allows the user to freely select a subset of the detector strain data (in both frequency and time domain), starting from a collection of basic files (BSD files) in a special data format. All the properties of the framework are described in Piccinni et al. (2018), and here we only remind the reader that the standard format of the BSD files, containing an opportunely down-sampled complex time series, covers a $10 \mathrm{~Hz}$ frequency band and $\sim 1$ month of data.
For the purpose of this search, where the actual signal frequency is unknown, each BSD file is partially corrected for the Doppler modulation in each $1 \mathrm{~Hz}$ frequency subband using its central frequency (see Piccinni et al. 2020 for more details). From this partially corrected time series, a collection of time-frequency peaks (called "peakmaps") is obtained, by choosing all the local maxima above a given threshold from equalized spectra (Astone et al. 2005). The equalization is given by the square modulus of the periodogram divided by the average spectrum. In this way also narrow peaks are kept. This peakmap is the input of the FH transform, which maps each time-frequency peak into the intrinsic source frequency and spin-down $\left(f_{0}, \dot{f}_{0}\right)$ plane at a given reference time. The resolution of a single $\mathrm{FH}$ map is the size of the bins in the template grid

$$
\begin{gathered}
\delta f_{\mathrm{FH}}=\frac{1}{T_{\mathrm{coh}} K_{f}}, \\
\delta \dot{f}_{\mathrm{FH}}=\frac{1}{T_{\mathrm{coh}} T_{\mathrm{obs}} K_{\dot{f}}},
\end{gathered}
$$

where $T_{\text {coh }}$ is the coherence time, while $T_{\text {obs }}$ is the observational time. The parameters $K_{f}$ and $K_{\dot{f}}$ are the overresolution factors as described in Astone et al. (2014b), here chosen as $K_{f}=10$ and $K_{\dot{f}}=2$. The coherence time $T_{\text {coh }}$ scales with the maximum frequency of the band as $1 / \sqrt{f_{\max }}$, and hence the frequency and spin-down bin sizes in Equations (1) and (2) change for each $10 \mathrm{~Hz}$ band. For a source with age $t_{\text {age, }}$, the 
Table 2

Sources Searched in the BSD Analysis (Section 4.1) and the Parameter Space Covered

\begin{tabular}{|c|c|c|c|c|}
\hline Source & Minimum $t_{\text {age }}(\mathrm{kyr})$ & $\begin{array}{c}T_{\text {coh }}(\mathrm{hr}) \\
(@ 100 \mathrm{~Hz})\end{array}$ & $f(\mathrm{~Hz})$ & $\begin{array}{l}\dot{f}\left(\mathrm{~Hz} \mathrm{~s}^{-1}\right) \\
(@ 100 \mathrm{~Hz})\end{array}$ \\
\hline G65.7+1.2, G189.1+3.0, G266.2-1.2 & 3 & 8 & $(10,600)$ & $\left(-1.06 \times 10^{-9}, 1.06 \times 10^{-10}\right)$ \\
\hline G353.6-0.7 & 27 & 8 & $(10,1000)$ & $\left(-1.17 \times 10^{-10}, 1.17 \times 10^{-11}\right)$ \\
\hline G18.9-1.1 & 4.4 & 8 & $(10,1000)$ & $\left(-7.13 \times 10^{-10}, 7.13 \times 10^{-11}\right)$ \\
\hline G39.2-0.3 & 4.7 & 8 & $(10,1000)$ & $\left(-6.75 \times 10^{-10}, 6.75 \times 10^{-11}\right)$ \\
\hline $\mathrm{G} 93.3+6.9$ & 5 & 8 & $(10,1000)$ & $\left(-6.34 \times 10^{-10}, 6.34 \times 10^{-11}\right)$ \\
\hline
\end{tabular}

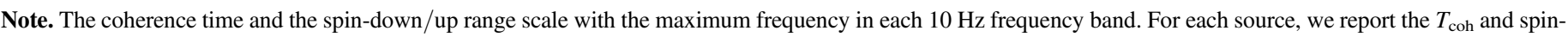
down/up range used for the frequency band $[90,100] \mathrm{Hz}$ where $f_{\max }=100 \mathrm{~Hz}$.

spin-down range is defined as $-f_{\max } / t_{\text {age }} \leqslant \dot{f} \leqslant 0.1 f_{\max } / t_{\text {age }}$, where $f_{\max }$ is the maximum frequency in each $10 \mathrm{~Hz}$ band. In this analysis, the age of the source affects the parameter space investigated, with a wider spin-down range covered when the source is younger. When possible, we use the youngest age estimate available in the SNRcat catalog (Ferrand \& SafiHarb 2012; SNR 2020). On the other hand, according to the age of the source, we can consider the effects of the secondorder spin-down as negligible or not (a discussion is reported in Appendix B.1). In this search, we investigate a frequency band of $[10,600] \mathrm{Hz}$ for targets with assumed $t_{\text {age }} \leqslant 3 \mathrm{kyr}$ and a wider range of $[10,1000] \mathrm{Hz}$ for older sources. We remind the reader of the subtle difference when talking about the source age estimates (which is most of the time inferred from the SNR age) and the characteristic age of the star (which is unknown because they have no observed electromagnetic pulsations). The maximum coherence time used is $17.8 \mathrm{hr}$ for the frequency band $[10,20] \mathrm{Hz}$ and a minimum of $2.5 \mathrm{hr}$ for $[990,1000] \mathrm{Hz}$. We search both positive and negative $\dot{f}$ to allow for the possibility of unexpected spin-up. A summary of the parameter space investigated for each source is shown in Table 2.

The first set of candidates is selected from a final FH map, which is the sum of all the single monthly based FH maps spanning the same frequency and spin-down ranges. These candidates are independently selected in each detector, including Virgo, using the ranking procedure of Astone et al. (2014b), where candidates with the highest FH number count are kept. At a later stage, coincidences are calculated between the candidate sets from the two LIGO detectors using a coincidence distance defined as

$$
d=\sqrt{\left(\frac{\Delta f}{\delta f_{\mathrm{FH}}}\right)^{2}+\left(\frac{\Delta \dot{f}}{\delta \dot{f}_{\mathrm{FH}}}\right)^{2}},
$$

where $\Delta f$ and $\Delta \dot{f}$ are the differences between the candidate parameters in each data set. A candidate is then selected when the coincidence distance is below a given threshold distance, $d_{\mathrm{thr}}$, in this search chosen equal to 4 . The choice of the window size has been widely discussed in Astone et al. (2014b), using injected simulated signals.

The coincidence step has been applied first to the pair of LIGO candidates. At a later stage, the same coincidence criterion has been applied between the HL coincident candidates and the most significant Virgo candidates. Candidates found in triple coincidence were discarded after applying the postprocessing methods described in Appendix A. However, we cannot conclude with certainty that a pair of LIGO candidates are nonastrophysical if they have $d<d_{\mathrm{thr}}$ but are not seen in Virgo data, because Virgo is less sensitive than LIGO. For this reason we also postprocessed all the candidates found in coincidence between $\mathrm{H}$ and $\mathrm{L}$ only.

Surviving candidates are further investigated through a follow-up process described in Appendix A. Also, we apply a threshold to the Critical Ratio (CR) $\rho_{\mathrm{CR}}$, which measures the statistical significance of a candidate based on the number count associated with the pixel of the FH map where the candidate lies. The threshold $\rho_{\mathrm{CR}, \text { thr }}$ is chosen as the mean $\rho_{\mathrm{CR}}$ plus one standard deviation of the $\mathrm{CR}$ distribution across the candidates excluding those due to known instrumental lines (Appendix A.1.1) and with an inconsistent significance among the two detectors (Appendix A.1.2). For the targets G65.7+1.2, $\mathrm{G} 189.1+3.0$, and $\mathrm{G} 266.2-1.2$, we use $\rho_{\mathrm{CR}, \mathrm{thr}}=4.7$; for G18.9-1.1 and G93.3+6.9, we use $\rho_{\mathrm{CR}, \mathrm{thr}}=4.6$; and for G353.6-0.7 and G39.2-0.3, we use $\rho_{\mathrm{CR}, \mathrm{thr}}=4.5$. The threshold chosen here is less stringent than in Piccinni et al. (2020), where the threshold was $\approx 6.5$, corresponding to the probability of picking an average of one false candidate over the total number of points in the parameter space, under the assumption of Gaussian noise. For this work, a lower CR threshold is picked since we are using some new postprocessing methods, described in Appendix A, which allow us to follow up a higher number of candidates, given the low computational cost of each step.

\subsection{Single-harmonic Viterbi}

An HMM is an efficient search algorithm capable of handling both spin-down and spin wandering. Previous searches for young SNRs using an HMM (Sun et al. 2018) were conducted in the Advanced LIGO O2 data, but no evidence for a GW signal was reported (Millhouse et al. 2020).

An HMM models a time-varying signal with underlying hidden (i.e., unobservable) parameters by treating the hidden parameters as links in a Markov chain, with each hidden parameter linked to an observable through a likelihood statistic. Given an observed sequence, the goal is to infer the most probable hidden sequence. For a set of $N_{T}$ observations at discrete times $\left\{t_{0}, t_{1}, \ldots, t_{N_{T}-1}\right\}$, the corresponding discrete states $\left\{q\left(t_{0}\right), q\left(t_{1}\right), \ldots, q\left(t_{N_{T}-1}\right)\right\}$ (chosen from $N_{Q}$ possible hidden states $\left.\left\{q_{1}, \ldots, q_{N_{Q}}\right\}\right)$ form a Markov chain with transition probabilities from $t_{k}$ to $t_{k+1}$ defined by $A_{q_{i} q_{j}}=$ $P\left[q\left(t_{k+1}\right)=q_{j} \mid q\left(t_{k}\right)=q_{i}\right]$. For this search, we choose $A_{q_{i} q_{i}}=A_{q_{i \pm 1} q_{i}}=1 / 3$ and all other $A_{q_{i} q_{j}}=0$, allowing the frequency to remain static or wander up or down one bin for each time step. This allows us to track both spin-down and stochastic spin wandering, which may cause spin-up. Strictly speaking, spin-down is expected to be more rapid than spin-up due 
Table 3

Sources Searched in the Single-harmonic Viterbi Analysis (Section 4.2) and the Parameter Space Covered

\begin{tabular}{lccccc}
\hline \hline Source & Minimum $t_{\text {age }}(\mathrm{kyr})$ & $D(\mathrm{kpc})$ & $T_{\text {coh }}(\mathrm{hr})$ & $f(\mathrm{~Hz})$ & $\dot{f}(\mathrm{~Hz} \mathrm{~s})$ \\
\hline G1.9+0.3 & 0.10 & 8.5 & 1.0 & $(31.56,121.7)$ & $\left(-3.858 \times 10^{-8}, 3.858 \times 10^{-8}\right)$ \\
G15.9+0.2 & 0.54 & 8.5 & 1.0 & $(44.03,657.1)$ & $\left(-3.858 \times 10^{-8}, 3.858 \times 10^{-8}\right)$ \\
G18.9-1.1 & 4.4 & 2 & 1.9 & $(31.02,1511)$ & $\left(-1.507 \times 10^{-8}, 1.507 \times 10^{-8}\right)$ \\
G39.2-0.3 & 3.0 & 6.2 & 2.8 & $(62.02,459.2)$ & $\left(-1.968 \times 10^{-8}, 1.968 \times 10^{-8}\right)$ \\
G65.7+1.2 & 20 & 1.5 & 4.7 & $(35.10,1128)$ & $\left(-3.149 \times 10^{-9}, 3.149 \times 10^{-9}\right)$ \\
G93.3+6.9 & 5.0 & 1.7 & 1.9 & $(30.00,1668)$ & $\left(-1.335 \times 10^{-8}, 1.335 \times 10^{-8}\right)$ \\
G111.7-2.1 & 0.30 & 3.3 & 1.0 & $(25.71,365.1)$ & $\left(-3.858 \times 10^{-8}, 3.858 \times 10^{-8}\right)$ \\
G189.1+3.0 & 3.0 & 1.5 & 1.4 & $(26.13,2000)$ & $\left(-1.968 \times 10^{-8}, 1.968 \times 10^{-8}\right)$ \\
G266.2-1.2 & 0.6 & 1.0 & $(18.36,839.6)$ & $\left(-3.858 \times 10^{-8}, 3.858 \times 10^{-8}\right)$ \\
G291.0-0.1 & 1.2 & 3.5 & 1.0 & $(31.97,1460)$ & $\left(-3.858 \times 10^{-8}, 3.858 \times 10^{-8}\right)$ \\
G330.2+1.0 & 1.0 & 0.9 & $(21.74,1939)$ & $\left(-3.858 \times 10^{-8}, 3.858 \times 10^{-8}\right)$ \\
G347.3-0.5 & 1.6 & 4.5 & $(31.96,730.1)$ & $\left(-3.858 \times 10^{-8}, 3.858 \times 10^{-8}\right)$ \\
G350.1-0.3 & 0.60 & 3.2 & 1.0 & $(77.86,318.3)$ & $\left(-3.858 \times 10^{-8}, 3.858 \times 10^{-8}\right)$ \\
G353.6-0.7 & 57 & 10 & $(25.72,121.7)$ & $\left(-2.295 \times 10^{-9}, 2.295 \times 10^{-9}\right)$ \\
G354.4+0.0 & 0.10 & 1.0 & & $\left(-3.858 \times 10^{-8}, 3.858 \times 10^{-8}\right)$ \\
\hline
\end{tabular}

Note. The parameter space for each of the 15 sources is derived using the age and distance estimates in the second and third columns.

to spin wandering, but the exact values of $A_{q_{i} q_{j}}$ have minimal effect on the performance of an HMM, provided they capture the behavior of the signal in a broad sense (Quinn \& Hannan 2001; Suvorova et al. 2016). We assume a uniform prior over the initial state, i.e., $\Pi\left[q\left(t_{0}\right)\right]=N_{Q}^{-1}$. The observations are denoted $\left\{o\left(t_{0}\right), o\left(t_{1}\right), \ldots, o\left(t_{N_{T}-1}\right)\right\}$ and are connected to $q\left(t_{k}\right)$ through unknown parameters. We call the probability of observing $o\left(t_{k}\right)$ given some state $q\left(t_{k}\right)$ the emission probability $L_{o\left(t_{k}\right) q\left(t_{k}\right)}=$ $P\left[o\left(t_{k}\right) \mid q\left(t_{k}\right)\right]$. Given some observed sequence $O$, we can then infer the most likely hidden sequence $Q^{*}$ by maximizing

$$
P\left(Q^{*} \mid O\right)=\Pi\left[q\left(t_{0}\right)\right] \prod_{k=1}^{N_{T}-1} L_{o\left(t_{k}\right) q\left(t_{k}\right)} A_{q\left(t_{k}\right) q\left(t_{k-1}\right)} .
$$

The Viterbi algorithm is an efficient implementation of the inference step, using dynamic programming to sample and discard unfavorable paths at each time step (Viterbi 1967; Suvorova et al. 2016).

For our purposes, the hidden state is the true GW frequency and the observable is the value of the $\mathcal{F}$-statistic, calculated coherently over a block of duration $T_{\text {coh }}$ and width (in the frequency domain) $\left(2 T_{\text {coh }}\right)^{-1}$. The $\mathcal{F}$-statistic is a maximum likelihood filter for a CW signal of frequency $f$ with time derivatives $\dot{f}, \ddot{f}$, etc. (for more details on the $\mathcal{F}$-statistic, please see Jaranowski et al. 1998). In this search, we compute the $\mathcal{F}$-statistic as a function of $f$ only and account for spin-down by choosing $T_{\text {coh }} \propto\left|\dot{f}_{0}^{\max }\right|^{-1 / 2}$ (as in Sun et al. 2018), where $\dot{f}_{0}^{\text {max }}$ is the maximum $\dot{f}$ within $T_{\text {coh }}$, such that the signal should wander by at most one frequency bin per time step.

We choose our parameter space according to the detectability of a potential signal. First, we estimate the maximum expected GW strain for a neutron star at distance $D$ with characteristic age $t_{\mathrm{age}}$ and a principle moment of inertia $I_{\mathrm{zz}}$ using

$$
\begin{aligned}
h_{0}^{\mathrm{age}}= & 2.27 \times 10^{-24}\left(\frac{1 \mathrm{kpc}}{D}\right)\left(\frac{1 \mathrm{kyr}}{t_{\mathrm{age}}}\right)^{1 / 2} \\
& \times\left(\frac{I_{\mathrm{zz}}}{10^{38} \mathrm{~kg} \mathrm{~m}^{2}}\right)^{1 / 2}
\end{aligned}
$$

and assuming purely gravitational spin-down (Wette et al. 2008). We also estimate the minimum detectable strain using an analytic estimate of the $95 \%$ confidence sensitivity for a semicoherent search, given by (Wette et al. 2008; Sun et al. 2018)

$$
h_{0}^{\text {est }}=\Theta S_{n}(f)^{1 / 2}\left(T_{\text {obs }} T_{\text {coh }}\right)^{-1 / 4} \text {, }
$$

where $S_{n}(f)$ is the noise amplitude spectral density. The statistical threshold $\Theta$ is defined by the location in parameter space and typically lies in the range $30 \lesssim \Theta \lesssim 40$. Following previous studies for CWs with an HMM, we take $\Theta=35$ (Wette et al. 2008; Sun et al. 2018). The frequency range for each source is defined by $h_{0}^{\text {est }}<h_{0}^{\text {age }}$. The parameter space for each source, including $T_{\text {coh }}$, is summarized in Table 3 , and the process for defining the parameter space is described in Appendix B.2.

We split the data into $N_{\text {band }}$ frequency subbands of width $2 \mathrm{~Hz}$ to ensure that loud, non-Gaussian noise artifacts (e.g., lines) are confined to one subband and do not affect the whole analysis. We overlap the frequency subbands by $0.57 \mathrm{~Hz}$, ensuring that any signal corresponding to a rapidly spinning down neutron star can always be contained in a single subband.

For each subband, we apply the Viterbi algorithm outlined above and obtain $N_{Q}$ frequency paths ending in $N_{Q}$ different bins with associated likelihoods $\mathcal{L}$. Alternative implementations of Viterbi (including Suvorova et al. 2016; Sun et al. 2018) used a Viterbi score as their detection statistic (see Section 4.3). This statistic generally requires $N_{T} \ll N_{Q}$. Millhouse et al. (2020) demonstrated that this statistic fails to identify an injected (or real) path for $N_{T} \sim N_{Q}$ because the score is calculated for the optimal path relative to other paths in the band. If most of the paths overlap, the optimal path is similar to other paths in the band. In this search, we have a minimum $T_{\text {coh }}=1 \mathrm{hr}\left(N_{T}=4391, N_{Q}=14,400\right)$, which is sufficient for almost one-third of Viterbi paths to converge over $T_{\text {obs }}$ and consequently lower than the sensitivity of the Viterbi score. To maintain the search sensitivity with $N_{T} \sim N_{Q}$, we use the loglikelihood $\mathcal{L}$ as our detection statistic. Using the process outlined in Appendix B.2, we determine the 1\% false-alarm 
Table 4

Sources Searched in the Dual-harmonic Viterbi Analysis (Section 4.3) and the Parameter Space Covered

\begin{tabular}{|c|c|c|c|c|}
\hline Source & Minimum $t_{\text {age }}(\mathrm{kyr})$ & $T_{\text {coh }}(\mathrm{hr})$ & $f_{\star}(\mathrm{Hz})$ & $\dot{f}_{\star}\left(\mathrm{Hz} \mathrm{s}^{-1}\right)$ \\
\hline G65.7+1.2 & 20 & 12 & $(50,338)$ & $\left(-1.34 \times 10^{-10}, 0\right)$ \\
\hline $\mathrm{G} 189.1+3.0$ & 20 & 12 & $(50,338)$ & $\left(-1.34 \times 10^{-10}, 0\right)$ \\
\hline G353.6-0.7 & 27 & 12 & $(50,457)$ & $\left(-1.34 \times 10^{-10}, 0\right)$ \\
\hline G18.9-1.1 & 4.4 & 9 & $(50,132)$ & $\left(-2.38 \times 10^{-10}, 0\right)$ \\
\hline G39.2-0.3 & 3 & 9 & $(50,90)$ & $\left(-2.38 \times 10^{-10}, 0\right)$ \\
\hline $\mathrm{G} 93.3+6.9$ & 5 & 9 & $(50,150)$ & $\left(-2.38 \times 10^{-10}, 0\right)$ \\
\hline G266.2-1.2 & 5.1 & 9 & $(50,153)$ & $\left(-2.38 \times 10^{-10}, 0\right)$ \\
\hline
\end{tabular}

threshold for each source and denote the corresponding likelihood $\mathcal{L}_{\text {th }}$. We follow up all unique frequency paths with $\mathcal{L}>\mathcal{L}_{\text {th }}$ using the procedure described in Appendix $\mathrm{A}$ and find no $\mathrm{CW}$ candidates that cannot be described by nonastrophysical noise.

\subsection{Dual-harmonic Viterbi}

Methods in Sections 4.1 and 4.2 assume that the star rotates about one of its principal axes of the moment of inertia, and hence the GWs are emitted at $2 f_{\star}$. This assumption is based on the fact that the phenomenon of free precession is not clearly observed in the population of known pulsars (Jones 2010). However, the superfluid interior of a star pinned to the crust along an axis nonaligned with any of its principal axes could allow the star to emit GWs at both $f_{\star}$ and $2 f_{\star}$, even without free precession (Jones 2010; Bejger \& Królak 2014; Melatos et al. 2015). The dual-harmonic emission mechanism motivates searches combining the two frequency components of a signal to improve signal-to-noise ratio. The HMM tracking scheme described in Section 4.2 has been extended to track two frequency components simultaneously (Sun et al. 2019). The signal model considered in this section consists of both $f_{\star}$ and $2 f_{\star}$ components, given by (Jaranowski et al. 1998; Sun et al. 2019)

$$
\begin{gathered}
h_{2+}=\frac{1}{2} h_{0}\left(1+\cos ^{2} \iota\right) \sin ^{2} \theta \cos 2 \Phi, \\
h_{2 \times}=h_{0} \cos \iota \sin ^{2} \theta \sin 2 \Phi, \\
h_{1+}=\frac{1}{8} h_{0} \sin 2 \iota \sin 2 \theta \sin \Phi, \\
h_{1 \times}=\frac{1}{4} h_{0} \sin \iota \sin 2 \theta \cos \Phi,
\end{gathered}
$$

where $\iota$ is the inclination angle of the source, $\theta$ is the wobble angle between the star's rotation axis and its principal axis of the moment of inertia, and $\Phi$ is the GW signal phase observed at the detector. In general, when precession and triaxiality of the star are included, emission occurs at other frequencies too (Zimmermann \& Szedenits 1979; Van Den Broeck 2005; Lasky \& Melatos 2013).

In this analysis, the HMM formulation generally follows the description in Section 4.2, with three major updates. First, two different coherent times of $T_{\text {coh }}=12$ and $9 \mathrm{hr}$ are selected for three sources with $t_{\text {age }} \gtrsim 20 \mathrm{kyr}$ and four sources with $t_{\text {age }} \lesssim$ $5 \mathrm{kyr}$, respectively. Second, two frequency components are tracked simultaneously. The GW signal for each frequency component is assumed to be monochromatic over $T_{\text {coh }}$. The signal power in each frequency bin is computed by the two- component $\mathcal{F}$-statistic, denoted by $\mathcal{F}_{1}\left(f_{i}\right)+\mathcal{F}_{2}\left(2 f_{i}\right)$, where $\mathcal{F}_{1}$ and $\mathcal{F}_{2}$ are the $\mathcal{F}$-statistic outputs computed in two separate frequency bands, and $f_{i}$ is the frequency value in the $i$ th bin. We use $\Delta f=1 /\left(4 T_{\text {coh }}\right)$ and $2 \Delta f=1 /\left(2 T_{\text {coh }}\right)$ as frequency bin sizes when computing $\mathcal{F}_{1}$ and $\mathcal{F}_{2}$, respectively, such that both the $f_{\star}$ and $2 f_{\star}$ signal components stay in one bin for each time interval $T_{\text {coh }}$. Third, we assume that the signal frequency evolution is dominated by secular spin-down and can be approximated by a negatively biased random walk. The unknown spin-down rate lies in the range between zero and the maximum estimated spin-down rate and can vary over time. Hence, we use a transition probability matrix $A_{q_{i-1} q_{i}}=A_{q_{i} q_{i}}=1 / 2$, with all other entries being zero. The full frequency band is divided into $1 \mathrm{~Hz}$ and $1.5 \mathrm{~Hz}$ subbands for $T_{\text {coh }}=12 \mathrm{hr}$ and $T_{\text {coh }}=9 \mathrm{hr}$, respectively, to parallelize computing. The detection statistic used in this analysis requires that the number of frequency bins in each subband (with bandwidth $B$ ) is significantly larger than the total number of tracking steps (i.e., $2 B T_{\text {coh }} \gg T_{\text {obs }} / T_{\text {coh }}$ ). Thus, for $T_{\text {coh }}=9 \mathrm{hr}$, we choose a $0.5 \mathrm{~Hz}$ wider subband such that the requirement is satisfied. More details are provided in Appendix B.3.

Seven sources in the top half of Table 1 with an assumed age of $t_{\text {age }} \gtrsim 3 \mathrm{kyr}$ are searched using this method. Due to the fact that two frequency bands are combined, this method is susceptible to noise features present in either band. Coherent times shorter than $\sim 5 \mathrm{hr}$ and, correspondingly, wider $\Delta f$ can further degrade the sensitivity. Hence, we do not search the other eight sources with $t_{\text {age }} \lesssim 3 \mathrm{kyr}$ that require a much shorter $T_{\text {coh. }}$. The parameter space covered for each source is listed in Table 4 . The $\dot{f}_{\star}$ range covered in this analysis is hence $\left|\dot{f}_{\star}\right| \in\left[0,1 /\left(4 T_{\text {coh }}^{2}\right)\right]$. The frequency range is determined as follows. For all seven sources, we fix the minimum frequency at 50 and $100 \mathrm{~Hz}$ for $f_{\star}$ and $2 f_{\star}$, respectively. We do not search below $50 \mathrm{~Hz}$ because the number of instrumental lines in each 1 $\mathrm{Hz}$ band significantly increases at low frequencies and the optimal Viterbi paths would be dominated by noise artifacts. The maximum frequency is set by the assumed minimum characteristic age of the source, $t_{\text {age }}$ (the second column in Table 4), assuming $\left|\dot{f}_{\star}\right|=f_{\star}(n-1)^{-1} t_{\text {age }}^{-1}$ (Sun et al. 2018; Abbott et al. 2019e), where $n=f_{\star} \ddot{f}_{\star} / \dot{f}_{\star}^{2}$ is the braking index, with $\ddot{f}_{\star}$ being the second time derivative of $f_{\star}$. We assume that the spin-down of the star is dominated by gravitational radiation due to a nonzero ellipticity, i.e., $n=5$.

We use the Viterbi score $S$ as the detection statistic in the dual-harmonic search, which indicates the significance of the optimal Viterbi path obtained in each subband compared to all other paths in that band at the final step of the tracking. Given that the condition $N_{T} \ll N_{Q}$ is generally satisfied with the choices of $T_{\text {coh }}$ in this method, the issue described in 
Section 4.2 with short $T_{\text {coh }} \sim 1$ hr does not happen. The full mathematical definition of $S$ is given in Sun et al. (2019). We determine a threshold corresponding to $1 \%$ false-alarm probability $S_{\mathrm{th}}=5.47$ and $S_{\mathrm{th}}=5.33$ for $T_{\mathrm{coh}}=12 \mathrm{hr}$ and $T_{\text {coh }}=9 \mathrm{hr}$, respectively, obtained from Monte Carlo simulations in Gaussian noise and verified in real O3a data. The results obtained from simulations in $\mathrm{O} 3 \mathrm{a}$ interferometric noise are consistent with the Gaussian noise thresholds.

\section{Sensitivity and Constraints}

A total of 42,464, 9236, and 477 first-stage candidates are identified across all SNRs in BSD, single-harmonic Viterbi, and dual-harmonic Viterbi pipelines. We apply a hierarchical veto procedure (Appendix A.1) to the full population and perform dedicated follow-up analyses on 35,1 , and 25 candidates for BSD, single-harmonic Viterbi, and dualharmonic Viterbi, respectively (Appendix A.2). No candidate survives from any pipeline. All are consistent with a nonastrophysical origin. In this section, we present the sensitivity of each pipeline and the constraints obtained from this analysis.

\subsection{BSD Constraints}

Surviving candidates are all compatible with noise fluctuations, and no evidence of their presence is found in Virgo O3a and/or in the full LIGO O3 data. We compute the constraints on the strain amplitude using a well-established method used in Piccinni et al. (2020) and described in Dreissigacker et al. (2018). The sensitivity curve is obtained from the $95 \%$ confidence level upper limits of 10 randomly selected frequency subbands of $1 \mathrm{~Hz}$ each for targets in the [10, $1000 \mathrm{~Hz}$ frequency band, and nine subbands for the remaining targets. The $h_{0}^{95 \%}$ in the subbands is computed with the frequentist approach, i.e., injecting 50 signals with a given amplitude $h_{0}$ and computing the corresponding detection efficiency. The injections are done for each source, assuming the same sky position as the selected source for each injection. The spin-down and polarization parameters $(\cos \iota$ and $\psi$ ) are randomly chosen from their uniform distributions. We repeat the injections in a given subband using 618 values of $h_{0}$ in the interval $\left[1.3 \times 10^{-26}, 3 \times 10^{-23}\right]$. The detection efficiency for a given amplitude $h_{0}$ is given by the fraction of injections recovered. The actual $h_{0}^{95 \%}$ corresponding to a detection efficiency of 0.95 is derived from the sigmoidal fit of the detection efficiency curve versus the injected amplitude.

Given that the sensitivity to $h_{0}$ is proportional to $\sqrt{S_{n}(f)}$, which is the noise amplitude spectral density, we compute the Normalized Upper Limit (NUL), $h_{\mathrm{NUL}}\left(f_{i}\right)=h_{0}^{95 \%}\left(f_{i}\right) / \sqrt{S_{n}\left(f_{i}\right)}$, in each of the randomly chosen subbands. We remark that it is the inverse of the more widely used "sensitivity depth" (Behnke et al. 2015). Since the NUL values should follow a linear trend, given by the dependence of the coherence time used in each $10 \mathrm{~Hz}$ band, we extrapolate the NUL values of the remaining bands with a linear fit of the NUL versus frequency. In this way we can translate the NUL values, interpolated from the linear fit for each $1 \mathrm{~Hz}$ band, into the $h_{0}^{95 \%}(f)$ curve. The final $h_{0}^{95 \%}(f)$ curve is then obtained for each detector, by multiplying the NUL values extrapolated from the linear fit in each $1 \mathrm{~Hz}$ band by the corresponding value of $\sqrt{S_{n}(f)}$ in that band, i.e.,

$$
h_{0}^{95 \%}(f)=h_{\mathrm{NUL}}(f) \sqrt{S_{n}(f)} .
$$

The sensitivity plots are presented in Figure 1, where we also report the indirect age-based limit from Equation (5) (solid line) for each target. The best sensitivity is below the indirect agebased limit for all the sources. In particular for $\mathrm{G} 65.7+1.2$, G189.1+3.0, and G266.21.2/Vela Jr., this happens for the full frequency band analyzed, except for the most disturbed regions, and for all the detectors. The difference in sensitivity among the analyzed targets is caused by the different antenna pattern response due to different sky locations of the sources, even when the same coherence time is used for multiple sources. We present different curves for each detector; the combined $h_{0}^{95 \%}(f)$ result would correspond to the one for the less sensitive LIGO detector. The best sensitivity at $95 \%$ confidence level occurs at the Livingston detector at $h_{0} \approx 7.8 \times$ $10^{-26}$ near $200 \mathrm{~Hz}$ for G65.7+1.2 and at $h_{0} \approx 7.7 \times 10^{-26}$ for G39.2-0.3 in the same bucket region.

\subsection{Single-harmonic Viterbi Constraints}

We report no evidence of CWs in the single-harmonic Viterbi search. In this section, we estimate the sensitivity of this search across 9 of the 15 sources. We estimate the sensitivity first using Equation (6) and assume that this is a reasonable representation of the key parameters determining the sensitivity, i.e., that between sources the sensitivity of the search is predominantly determined by $T_{\text {coh. }}$. So we determine the sensitivity for $T_{\text {coh }}=1 \mathrm{hr}$ using G266.2-1.2 and G347.3-0.5 and assume that the variation in sky position for other targets with the same $T_{\text {coh }}$ has a negligible effect on sensitivity. This assumption has been validated through detailed simulations. For each source we set limits on, we inject 100 simulated signals with fixed $h_{0}$ and randomly select $f$ and $\dot{f}_{0}$ into five frequency subbands, selected at random from a set of bands with no known lines, and which returned $<2$ unique paths with $\mathcal{L}>\mathcal{L}_{\text {th }}$ in the original search. We then apply the Viterbi algorithm to each injection. We repeat this for 5-10 values of $h_{0}$. Each set of $N_{I}=100$ injections forms a binomial distribution, with each injection and search acting as a Bernoulli trial with a probability of success (efficiency) $p$. We infer the value of $p$ given $s$ successes for each $h_{0}$ given using the Wilson interval (Wilson 1927)

$$
\begin{aligned}
p \approx & \frac{s+\frac{1}{2}\left(1-\alpha_{F} / 2\right)^{2}}{N_{I}+\left(1-\alpha_{F} / 2\right)^{2}} \\
& \pm \frac{1-\alpha_{F} / 2}{N_{I}+\left(1-\alpha_{F} / 2\right)^{2}} \sqrt{\frac{s\left(N_{I}-s\right)}{N_{I}}+\frac{\left(1-\alpha_{F} / 2\right)^{2}}{4}},
\end{aligned}
$$

where $\alpha_{F}$ is the false-alarm probability. For each frequency band, we fit a sigmoid curve (as in Banagiri et al. 2019) to the set of $h_{0}$ and the corresponding $p$ using the Bayesian inference package Bilby (Ashton et al. 2019) with a uniform prior over the sigmoid parameters. We sample the posterior and, for each sample, determine the $h_{0}^{95 \%}$ as the $h_{0}$ corresponding to $p=95 \%$. We take the average $h_{0}^{95 \%}$ of this population to be the $95 \%$ frequentist confidence upper limit in that frequency band. For each frequency band, we calculate $a=h_{0}^{95 \%} / h_{0}^{\text {est }}$ at the appropriate frequency, where $h_{0}^{\text {est }}$ is estimated by Equation (6). Lastly, we find the mean 


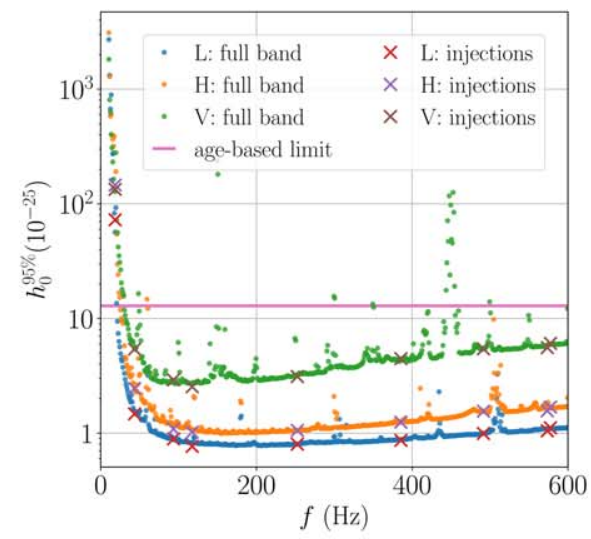

(a) $\mathrm{G} 65.7+1.2$

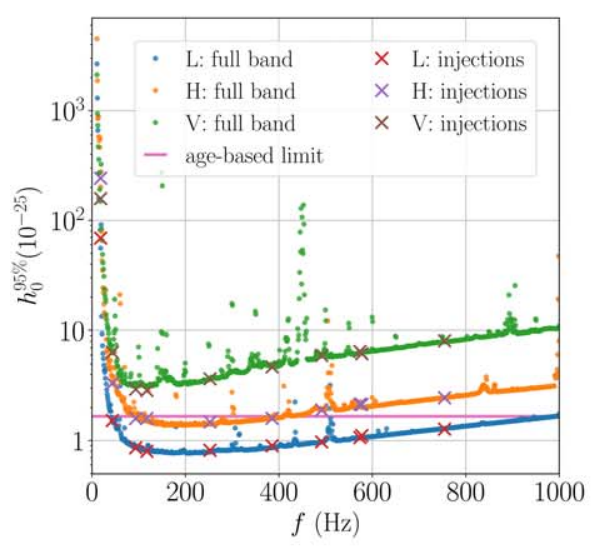

(d) G39.2-0.3

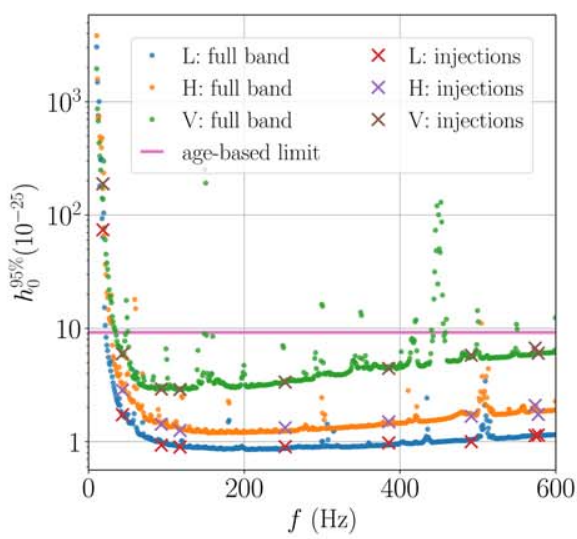

(b) $\mathrm{G} 189.1+3.0$

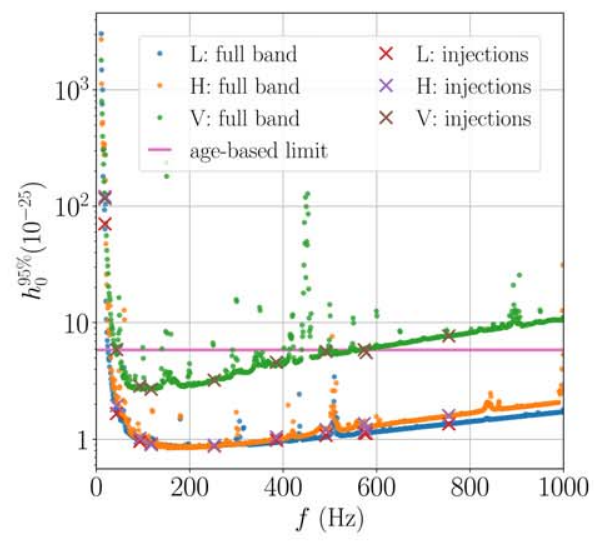

(e) G93.3+6.9

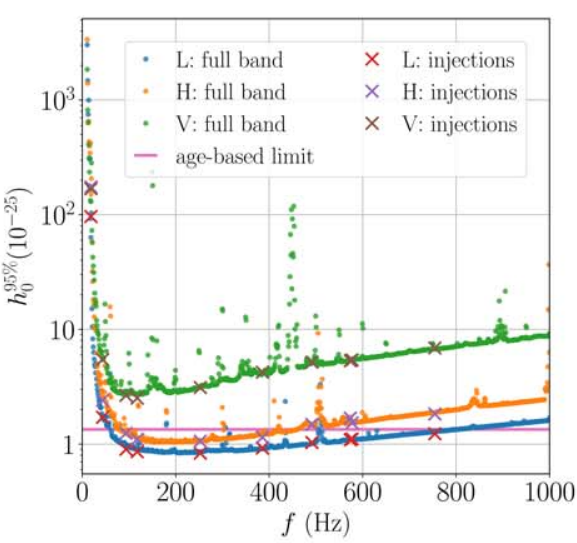

(g) G353.6-0.7

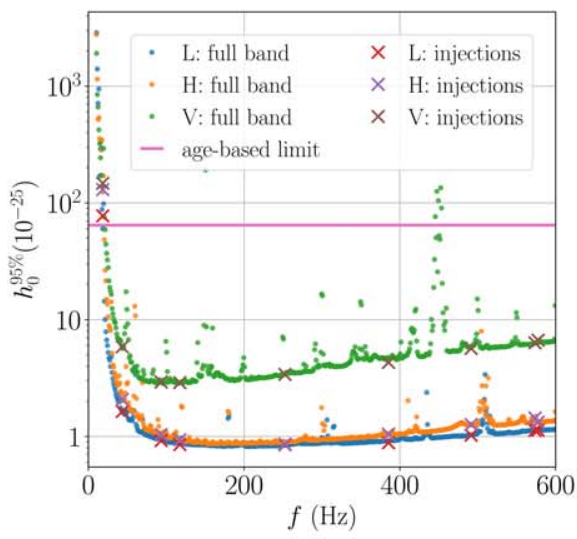

(c) G266.2-1.2/Vela Jr.

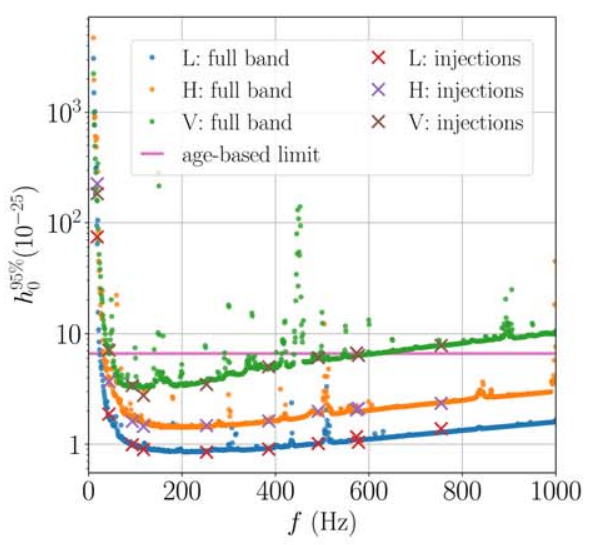

(f) G18.9-1.1

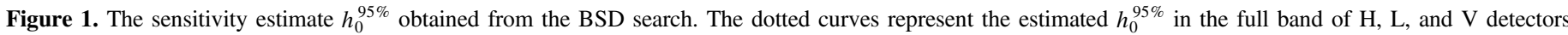

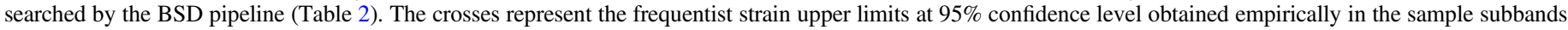

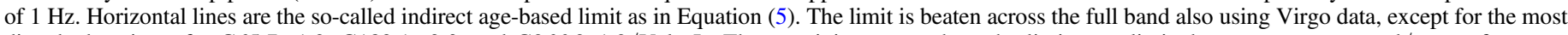

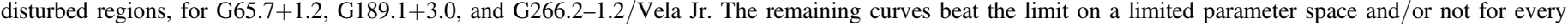
detector.

$a$ across the five frequency bands and calculate the sensitivity across the full frequency band as $h_{0}^{95 \%}=a h_{0}^{\text {est }}$, plotted as the curves in Figure 2. We overplot the age-based limit from Equation (5) (dashed line) for each target. Our search is more sensitive than the age-based limit for all targets except G18.9-1.1, G39.2-0.3, G330.2+1.0, and G353.6-0.7, despite G353.6-0.7 having the smallest detectable strain in this search, $2.64 \times 10^{-25}$ at $172 \mathrm{~Hz}$. The targets with the poorest overall sensitivity (those with short $T_{\text {coh }}$ ) place the tightest constraints relative to the agebased spin-down limit.

The constraints obtained in this search are for a random-walk signal model including spin-down and spin wandering. The random-walk signal model (including spin-down and spin wandering) and the range of $\dot{f}_{0}$ searched (up to $\dot{f}_{0}^{\max }=3.9 \times 10^{-8}$ 


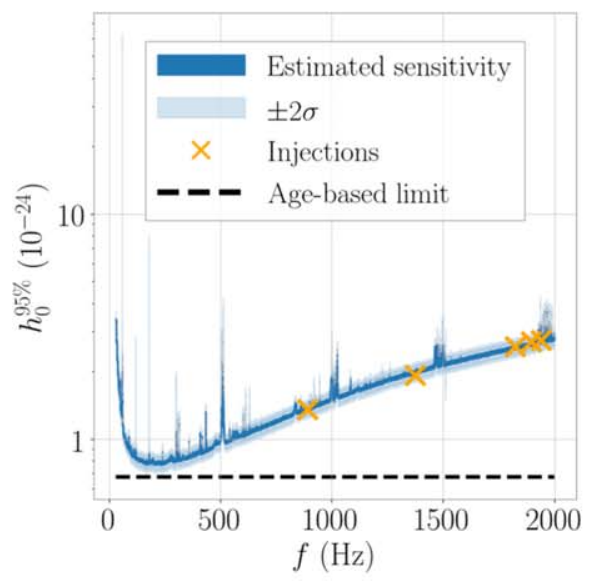

(a) G18.9-1.1

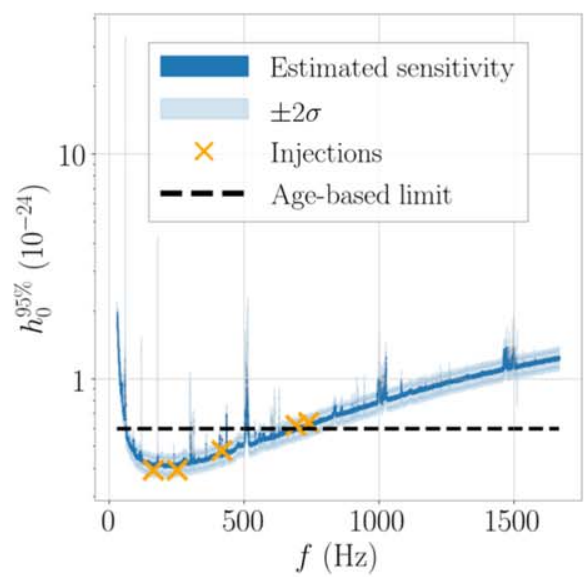

(d) $\mathrm{G} 93.3+6.9$

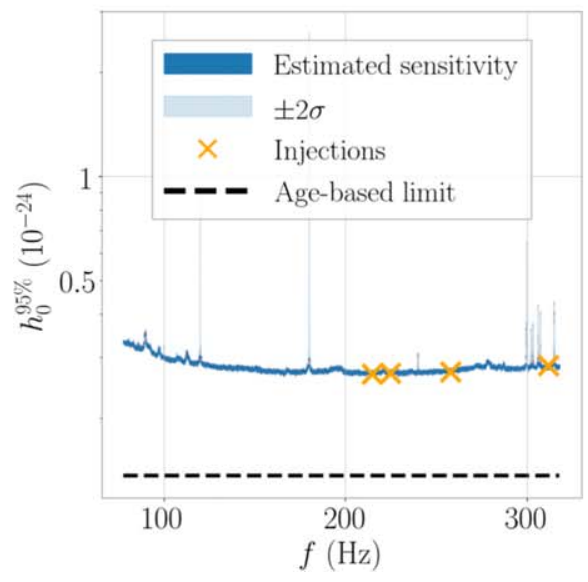

(g) G353.6-0.7

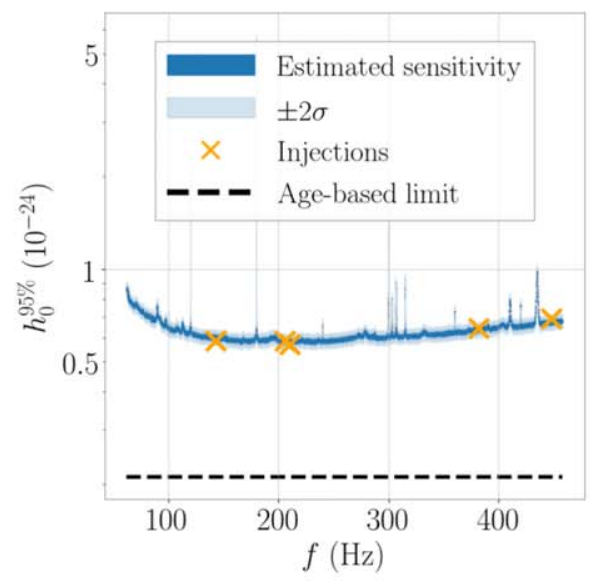

(b) G39.2-0.3

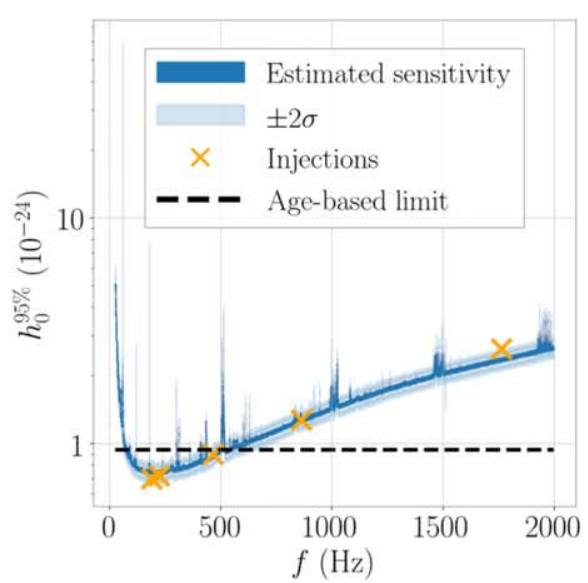

(e) G189.1+3.0

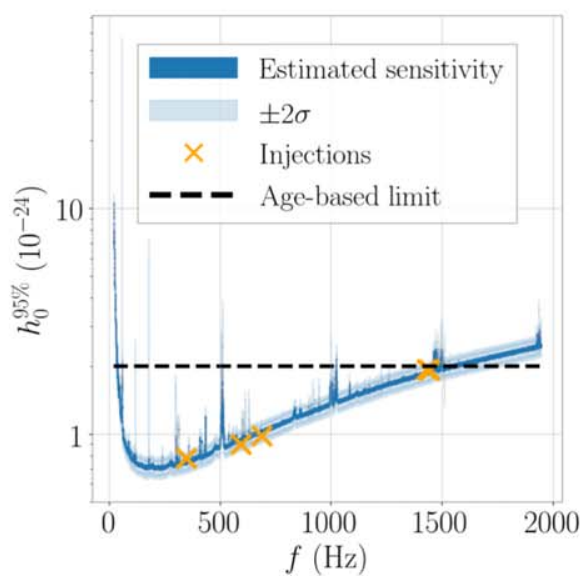

(h) G347.3-0.5

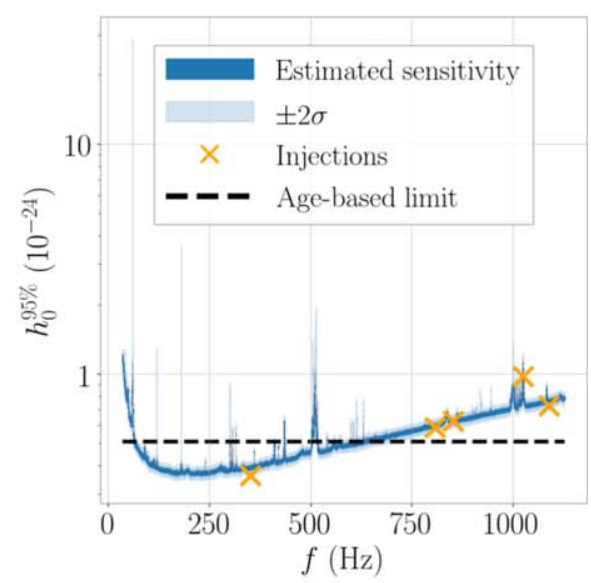

(c) $\mathrm{G} 65.7+1.2$

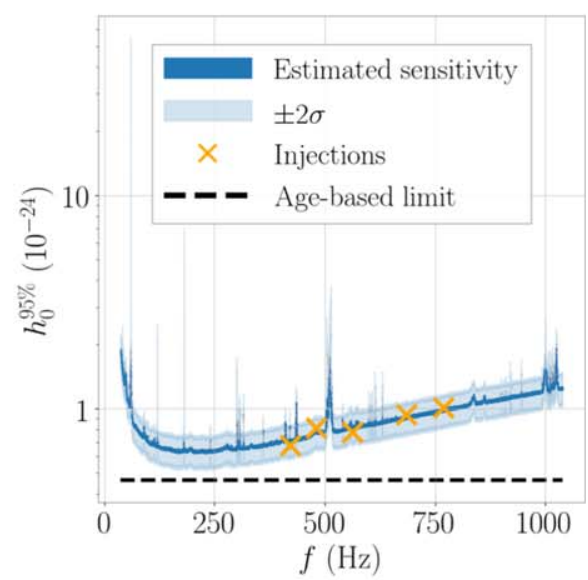

(f) $\mathrm{G} 330.2+1.0$

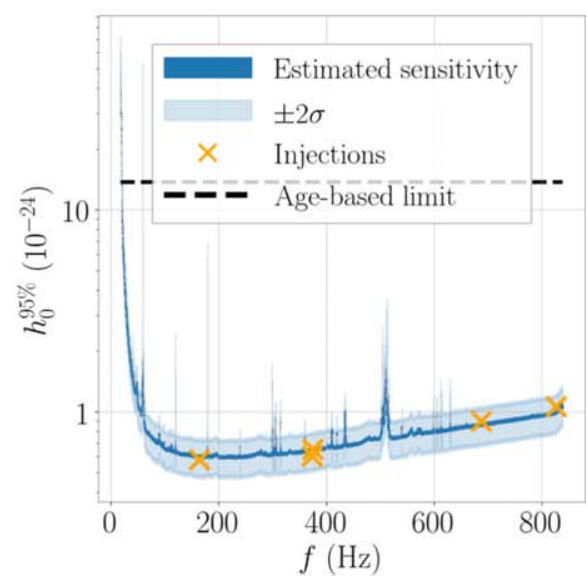

(i) G1.9+0.3, G15.9+0.2, G111.7-2.1, G266.2-1.2, G291.0-0.1, G350.1-0.3, G354.4+0.0

Figure 2. The sensitivity estimate $h_{0}^{95 \%}$ obtained from the single-harmonic Viterbi search for each source. Multiple sources have $T_{\text {coh }}=1 \mathrm{hr}$ and have the same sensitivity; these sources are shown on one plot for a representative source, G266.2-1.2. The blue curves represent the estimated $h_{0}^{95 \%}$ in the full band searched by the single-harmonic Viterbi pipeline (Table 3). The orange crosses represent the $h_{0}^{95 \%}$ values obtained empirically in the sample subbands. The black dashed line is the age-based upper limit on the GW strain from Equation (5).

$\mathrm{Hz} \mathrm{s}^{-1}$ for $T_{\text {coh }}=1 \mathrm{hr}$ ) mean that the $h_{0}^{95 \%}$ for this search is less stringent than for the other pipelines in this and other papers, which use a different signal model (e.g., Taylor expansion) and smaller range of $\dot{f}$. For G65.7+1.2, one of the injections at just over $1000 \mathrm{~Hz}$ appears to be on a noise spike despite known noise features being filtered out; however, the scale factor obtained for that band is consistent with the other four bands tested.

\subsection{Dual-harmonic Viterbi Constraints}

No evidence of CWs is found in the dual-harmonic Viterbi search. We empirically derive the sensitivity by estimating the 


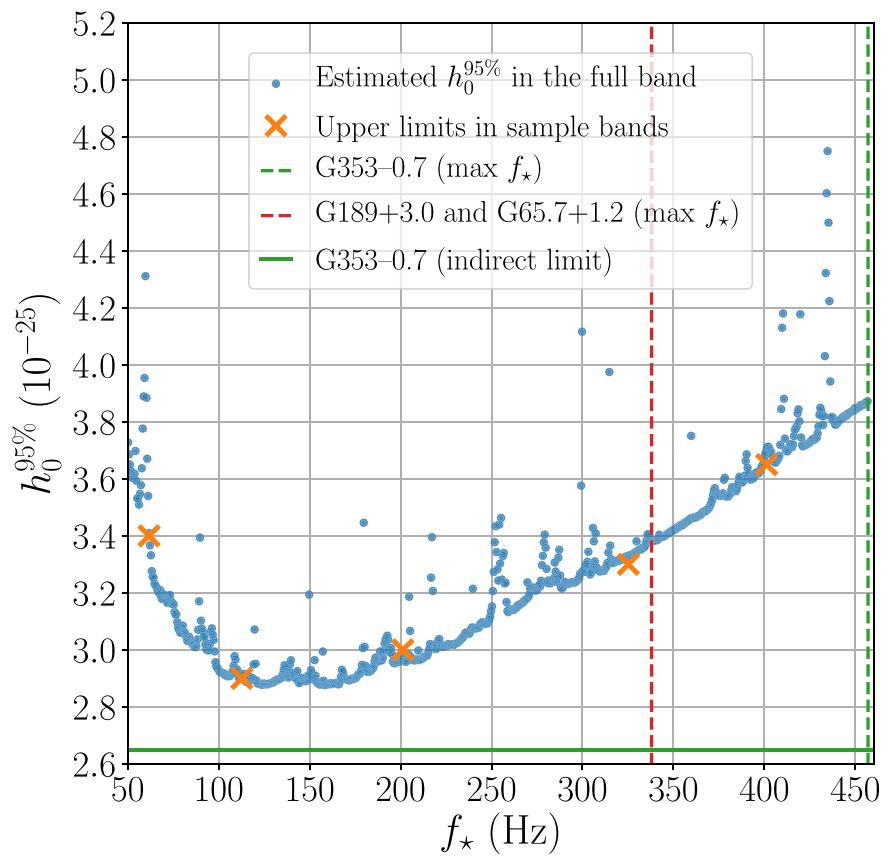

(a) G65.7+1.2, G189.1+3.0, G353.6-0.7

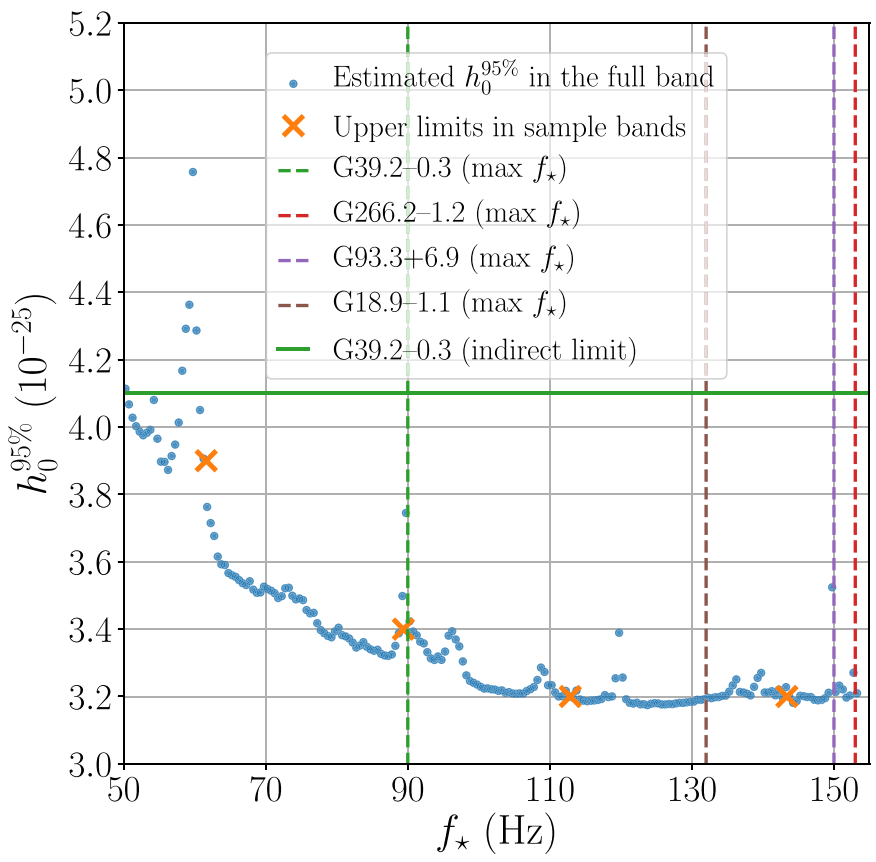

(b) G18.9-1.1, G39.2-0.3, G93.3+6.9, G266.2-1.2

Figure 3. The estimated sensitivity $h_{0}^{95 \%}$ obtained from the dual-harmonic Viterbi search as a function of $f_{\star}$ for (a) $T_{\text {coh }}=12 \mathrm{hr}$ and (b) $T_{\text {coh }}=9 \mathrm{hr}$, assuming a specific scenario with source properties $\theta=45^{\circ}$ and $\cos \iota=0$ (signals at both $f_{\star}$ and $2 f_{\star}$ are linearly polarized). The blue circles represent the estimated $h_{0}^{95 \%}$ in the full band searched by the dual-harmonic Viterbi pipeline (Table 4). The orange crosses represent the $h_{0}^{95 \%}$ strain upper limits obtained from injections in the randomly selected sample subbands. These estimates are obtained from randomized sky positions and hence apply to all sources using the same $T_{\text {coh. }}$ The vertical dashed lines indicate the maximum $f_{\star}$ covered for each SNR. The horizontal lines indicate the age-based indirect strain limits $h_{0}^{\text {age }}$ derived for the dual-harmonic signal model when $\theta=45^{\circ}$. For G353-0.7, the $h_{0}^{95 \%}$ obtained from the search has not beaten the indirect limit. For G39.2-0.3, the $h_{0}^{95 \%}$ has beaten the indirect limit at most of the frequencies except for the noisy bands around $60 \mathrm{~Hz}$. For all other sources, the $h_{0}^{\text {age }}$ values are much larger than $h_{0}^{95 \%}$ across the full band and thus are not shown in the figure.

signal strain $h_{0}^{95 \%}$ in each frequency subband (as a function of $f_{\star}$ ), such that a signal with $h_{0} \geqslant h_{0}^{95 \%}$ can be detected on $95 \%$ or more occasions. Since this pipeline considers a signal model with both $f_{\star}$ and $2 f_{\star}$ components, we use $f_{\star}$ instead of the GW frequency to avoid confusion. Note that the sensitivity on the strain $h_{0}$ quoted in this pipeline is based on a different signal model from the other two pipelines (see Equations (7)-(10)). Here we assume the special scenario $\theta=45^{\circ}$ and $\cos \iota=0$, i.e., signals at both $f_{\star}$ and $2 f_{\star}$ are linearly polarized. In this scenario, tracking the two frequency bands simultaneously offers the most significant sensitivity improvement from searching a single band, compared to other choices of $\theta$ and $\cos \iota$ (Sun et al. 2019).

Figure 3 shows $h_{0}^{95 \%}$ in all subbands and a set of frequentist upper limits obtained through injections in a handful of randomly selected sample subbands (orange crosses). The procedure to produce these results is as follows. First, we derive the frequentist upper limit in one sample subband, starting from $112.5 \mathrm{~Hz}$ for $f_{\star}$ and $225 \mathrm{~Hz}$ for $2 f_{\star}$. A set of 200 synthetic signals are injected into the O3a data at random sky positions in that subband with a fixed $h_{0}$. We use fixed $\theta=45^{\circ}$ and $\cos \iota=0$. The other source parameters, including $f_{\star}, \dot{f}_{\star}$, the polarization angle, and the initial phase, are randomly drawn from their uniform distributions. The corresponding detection rate is calculated. This process is repeated with different $h_{0}$ values with step size $1 \times 10^{-26}$ and $2 \times 10^{-26}$ in the regions where the detection rate is roughly above and below $50 \%$, respectively. With all the injected $h_{0}$ values and the corresponding detection rates, $h_{0}^{95 \%}$ is obtained through a sigmoidal fit. The $h_{0}^{95 \%}$ value found in the sample subband is $2.9 \times 10^{-25}$ and $3.2 \times 10^{-25}$ for $T_{\text {coh }}=12$ and $9 \mathrm{hr}$, respectively. Next, we use these values obtained in the sample subband (starting from $112.5 \mathrm{~Hz}$ for $f_{\star}$ and $225 \mathrm{~Hz}$ for $2 f_{\star}$ ) to analytically calculate $h_{0}^{95 \%}$ in the full frequency band (blue circles), using the scaling (Sun et al. 2019)

$$
h_{0}^{95 \%}(f) \propto\left(\frac{S_{n}(f) S_{n}(2 f)}{S_{n}(f)+S_{n}(2 f)}\right)^{1 / 2},
$$

where $S_{n}$ is the effective power spectral density calculated from the harmonic mean of the two detectors over all the 30-minute SFTs collected from 2019 September 1 to October 1 (GPS time 1251331218-1253923218). Finally, in order to verify the analytical scaling, the simulation procedure in the first step is repeated in several other randomly selected subbands, indicated by the orange crosses. The $h_{0}^{95 \%}$ values obtained empirically in those sample subbands agree to $<1.5 \%$ with the analytic sensitivity estimates. In the full frequency band searched, the best $h_{0}^{95 \%}$ values for $T_{\text {coh }}=12$ and $9 \mathrm{hr}$ are $2.88 \times 10^{-25}$ at $f_{\star}=$ $158.75 \mathrm{~Hz}$ and $3.17 \times 10^{-25}$ at $f_{\star}=123.75 \mathrm{~Hz}$, respectively. These results are obtained from randomized sky positions and hence apply to all sources using the same $T_{\text {coh }}$. In the dualharmonic Viterbi pipeline, the sensitivity is dominated by the length of $T_{\text {coh }}$ (for a fixed $T_{\text {obs }}$ ) rather than the sky position of the source. Additional spot checks validate that the difference between the empirical $h_{0}^{95 \%}$ values obtained from a fixed sky location and those from randomized sky positions is negligible. 


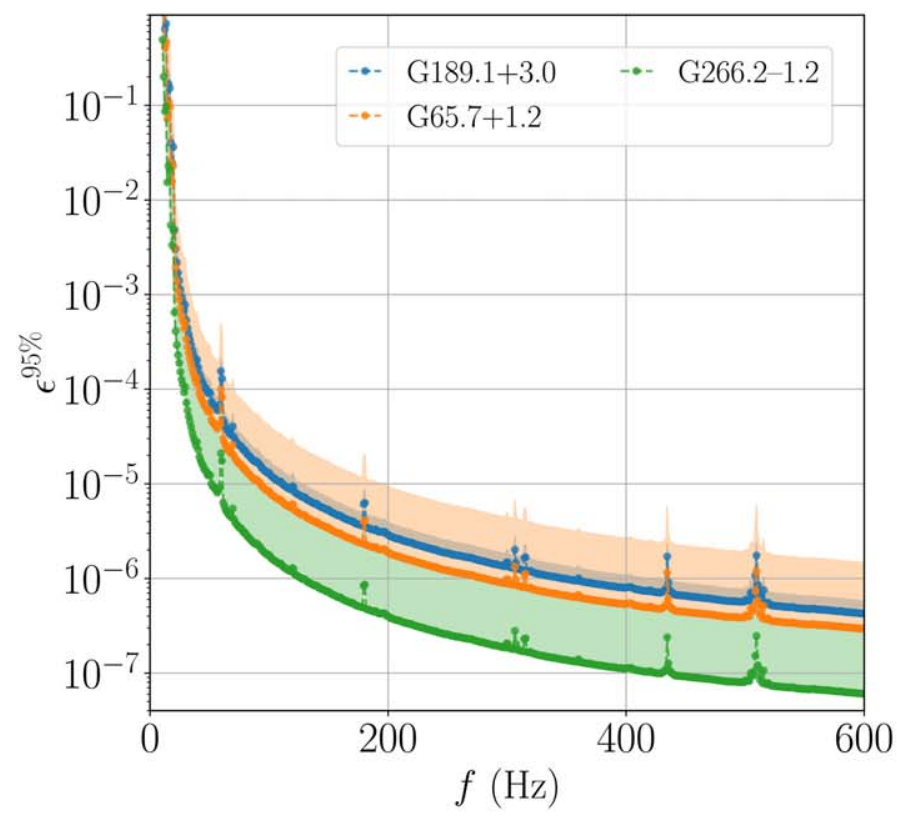

(a)

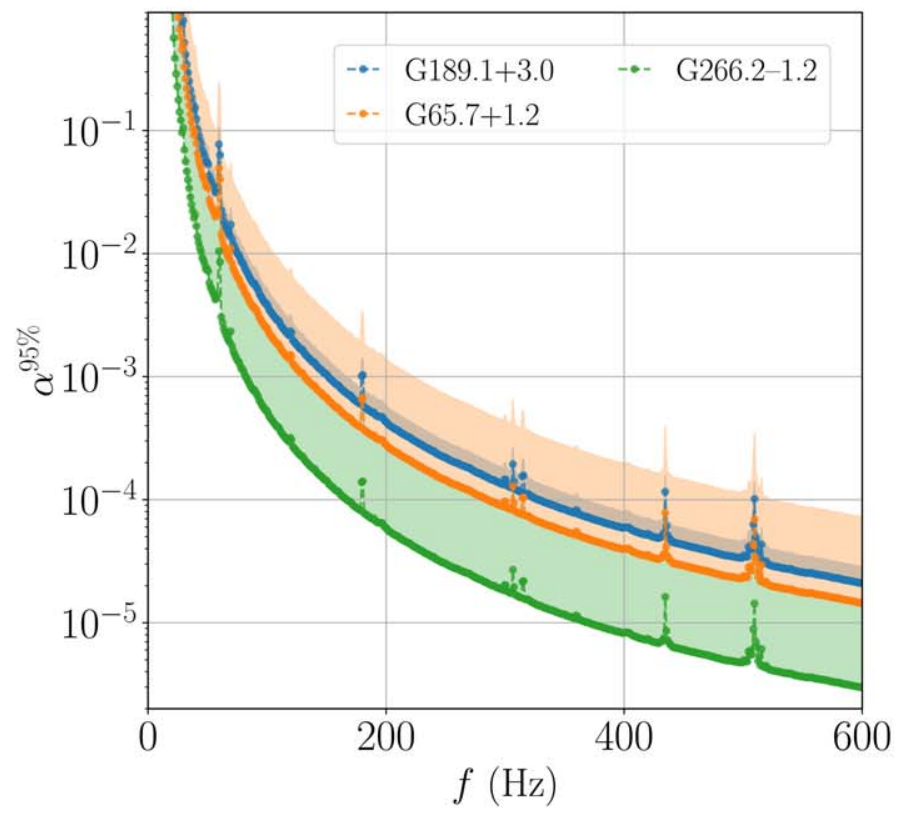

(c)

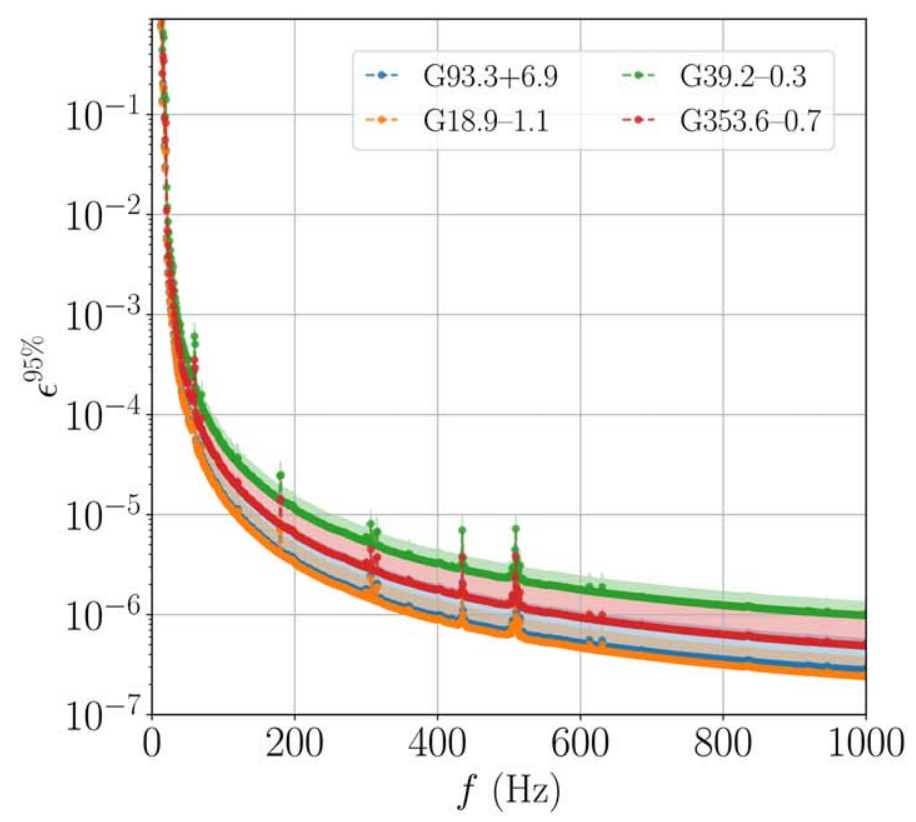

(b)

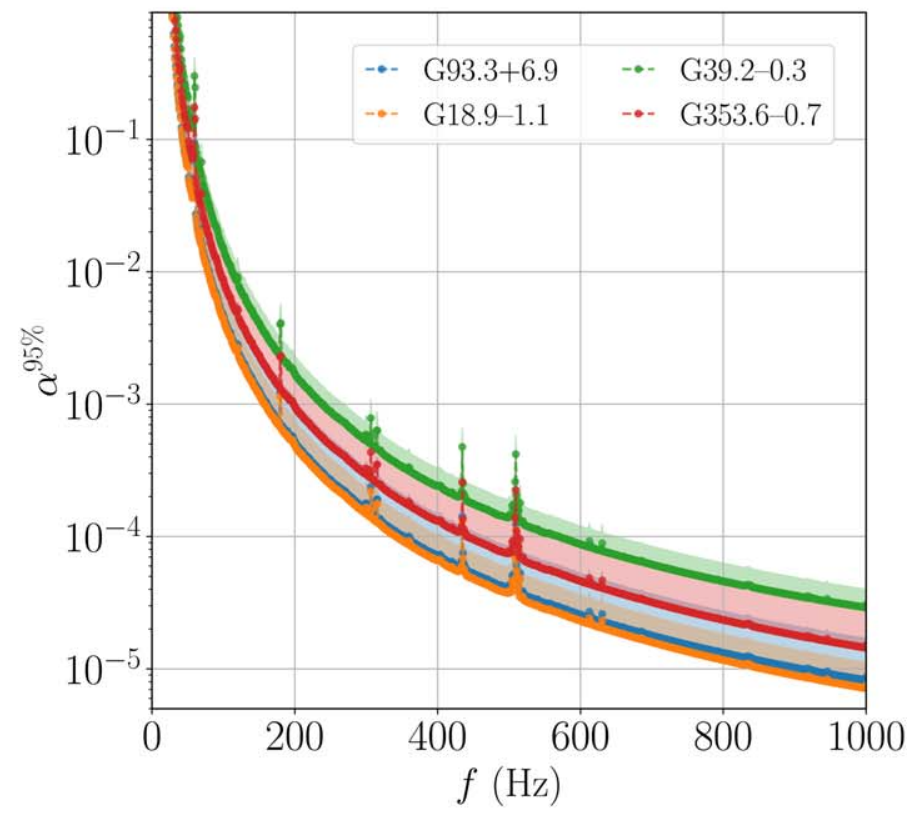

(d)

Figure 4. Constraints on the (a, b) neutron star ellipticity $\epsilon^{95 \%}$ and (c, d) $r$-mode amplitude $\alpha^{95 \%}$, from the BSD pipeline, converted from the $h_{0}^{95 \%}$ values in Figure 1 . Panels (a) and (c) report the results derived for G189.1+3.0, G65.7+1.2, and G266.2-1.2 (Vela Jr.), covering the [10, 600] Hz frequency band. Panels (b) and (d) report the results for G93.3+6.9, G18.9-1.1, G39.2-0.3, and G353.6-0.7, where the [10, 1000] Hz frequency band is investigated. Curves have been converted from $h_{0}^{95 \%}$ derived for the $\mathrm{L}$ detector. Shaded regions correspond to the inferred ellipticity and $r$-mode amplitude using the full range of distances in Table 1 . The minimum distance is assumed for the filled circle curves.

Assuming that the star's rotational kinetic energy loss is all radiated in GWs, the age-based indirect strain limits $h_{0}^{\text {age }}$ can be calculated for each source by fixing $\theta=45^{\circ}$ (consistent with the scenario presented in Figure 3), setting $t_{\mathrm{age}}$ to the value in Table 4, and setting the distance to the minimum value in Table 1. Note that the $h_{0}^{\text {age }}$ value derived explicitly for the dual-harmonic model with $\theta=45^{\circ}$ is a factor of $\sim 2$ larger than the value calculated from Equation (5) (Zimmermann \& Szedenits 1979; Wette et al. 2008). For five out of the seven sources, the indirect limits are much larger than the constraints obtained in this search across the full band. For G39.2-0.3, the $h_{0}^{95 \%}$ has beaten the indirect limit at most of the frequencies except for the noisy bands around $60 \mathrm{~Hz}$. For G353-0.7, the $h_{0}^{95 \%}$ obtained from the search is close to $h_{0}^{\text {age }}$ but has not reached it at any frequency. We emphasize that the sensitivity in the dual-harmonic Viterbi pipeline, and whether it beats the indirect limit, is not directly comparable to other methods owing to the model difference. 


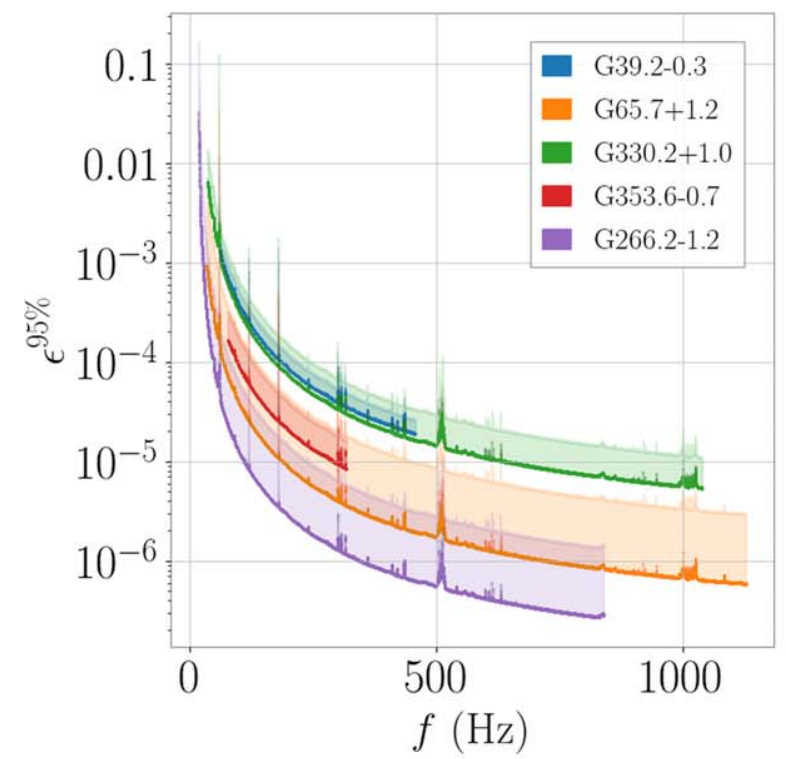

(a) Ellipticity constraints for targets with $f_{\max }<1500 \mathrm{~Hz}$

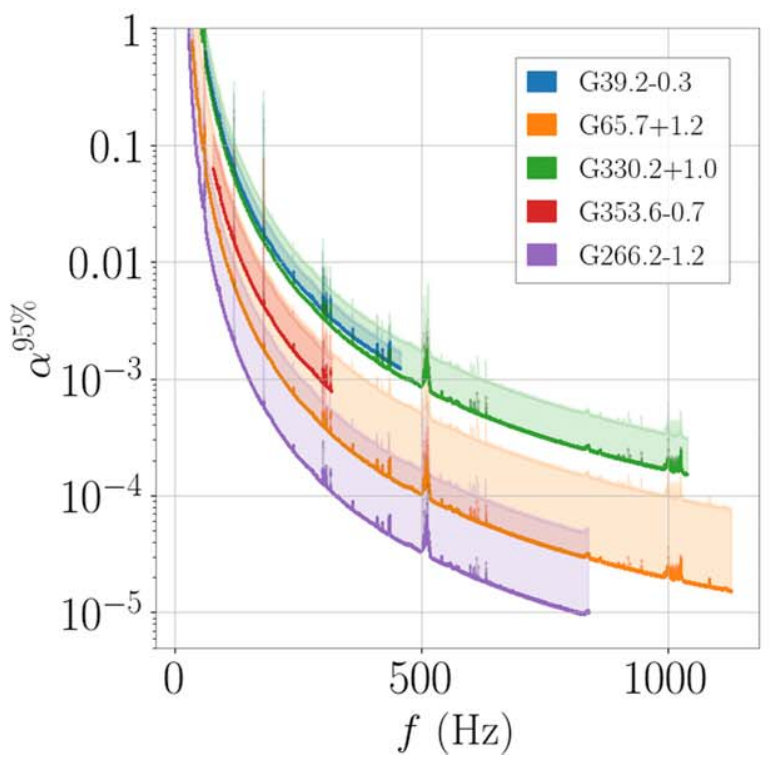

(c) r-mode constraints for targets with $f_{\max }<1500 \mathrm{~Hz}$

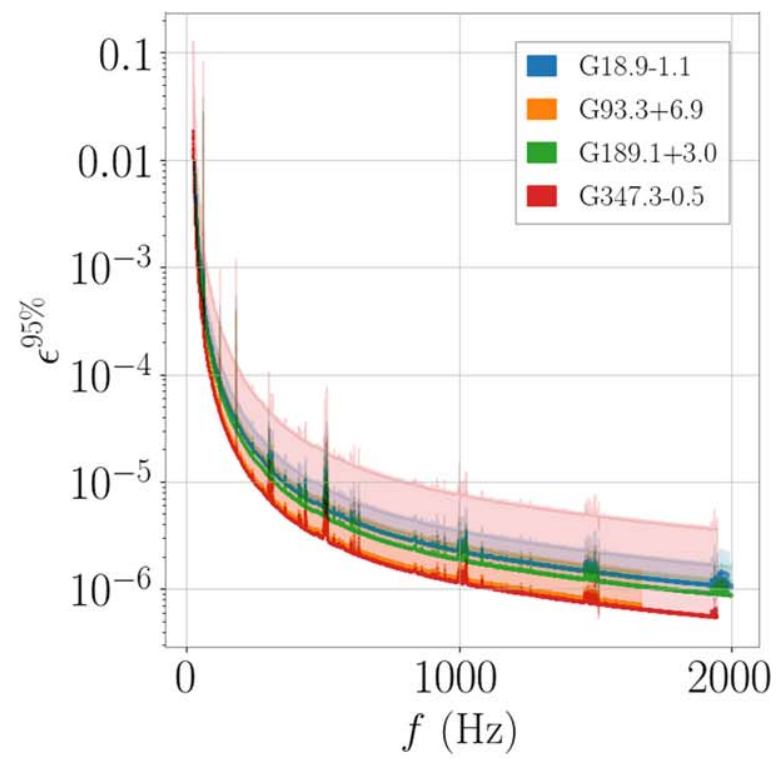

(b) Ellipticity constraints for targets with $f_{\max }>1500 \mathrm{~Hz}$

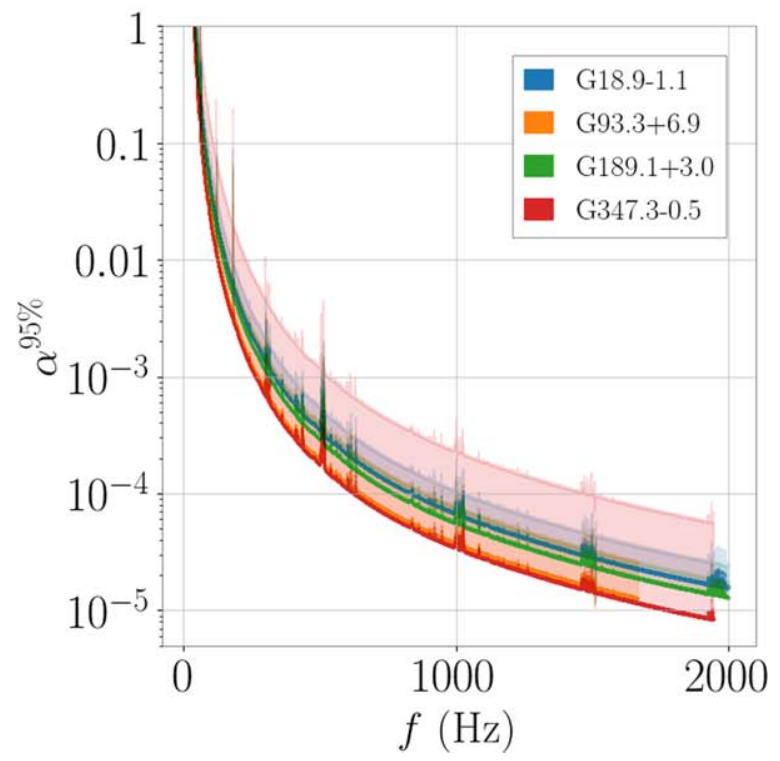

(d) r-mode constraints for targets with $f_{\max }>1500 \mathrm{~Hz}$

Figure 5. Constraints on the neutron star ellipticity (panels (a) and (b)) and $r$-mode amplitude (panels (c) and (d)) from the single-harmonic Viterbi pipeline converted from the $h_{0}^{95 \%}$ values in Figure 2. The results are plotted as a function of the GW frequency $f$. The solid line uses the closest distance estimate in Table 1, and the shaded area indicates the results across the full distance range. Panels (a) and (c) display the results for targets with $f_{\max }<1500 \mathrm{~Hz}$; panels (b) and (d) display results for targets with $f_{\max }>1500 \mathrm{~Hz}$.

\subsection{Astrophysical Implications}

The sensitivity in terms of the GW strain amplitude can be converted into constraints on the fiducial ellipticity of the neutron star, $\epsilon$ (Jaranowski et al. 1998), and the $r$-mode amplitude parameter, $\alpha$ (Owen 2010). We first discuss the constraints obtained in the BSD and single-harmonic Viterbi pipelines. For ellipticity, we assume that the GW frequency $f$ (equivalent to $f_{0}$ in the single-harmonic Viterbi pipeline) is at $2 f_{\star}$, which aligns with the model of a perpendicular biaxial rotor considered in both pipelines. Given $h_{0}^{95 \%}$ (derived with a uniform prior on the $\cos \iota$ ), we constrain the ellipticity of the neutron star in terms of the GW frequency $f=2 f_{\star}$ via (Jaranowski et al. 1998)

$$
\epsilon=9.46 \times 10^{-5}\left(\frac{h_{0}}{10^{-24}}\right)\left(\frac{D}{1 \mathrm{kpc}}\right)\left(\frac{100 \mathrm{~Hz}}{f}\right)^{2},
$$

assuming that the moment of inertia with respect to the rotation axis $\left(I_{z z}\right.$ for a perpendicular biaxial rotor) is $10^{38} \mathrm{~kg} \mathrm{~m}^{2}$. We can also convert $h_{0}^{95 \%}$ to limits on the amplitude of $r$-mode oscillations via (Owen 2010)

$$
\alpha \simeq 0.028\left(\frac{h_{0}}{10^{-24}}\right)\left(\frac{D}{1 \mathrm{kpc}}\right)\left(\frac{100 \mathrm{~Hz}}{f}\right)^{3} .
$$




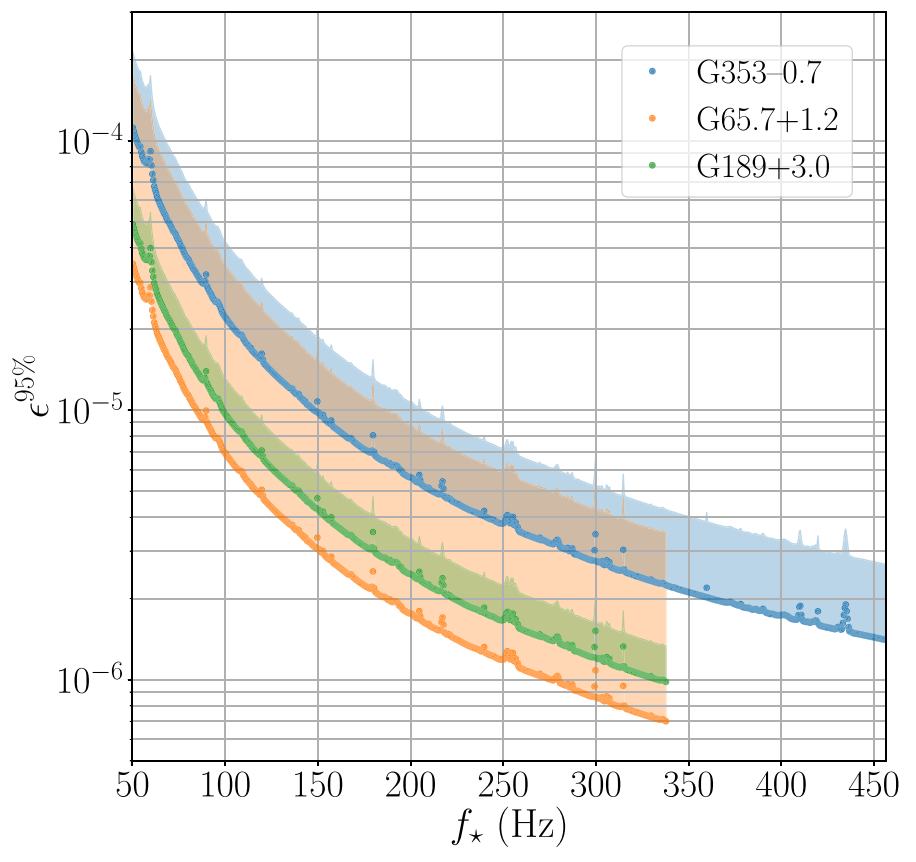

(a)

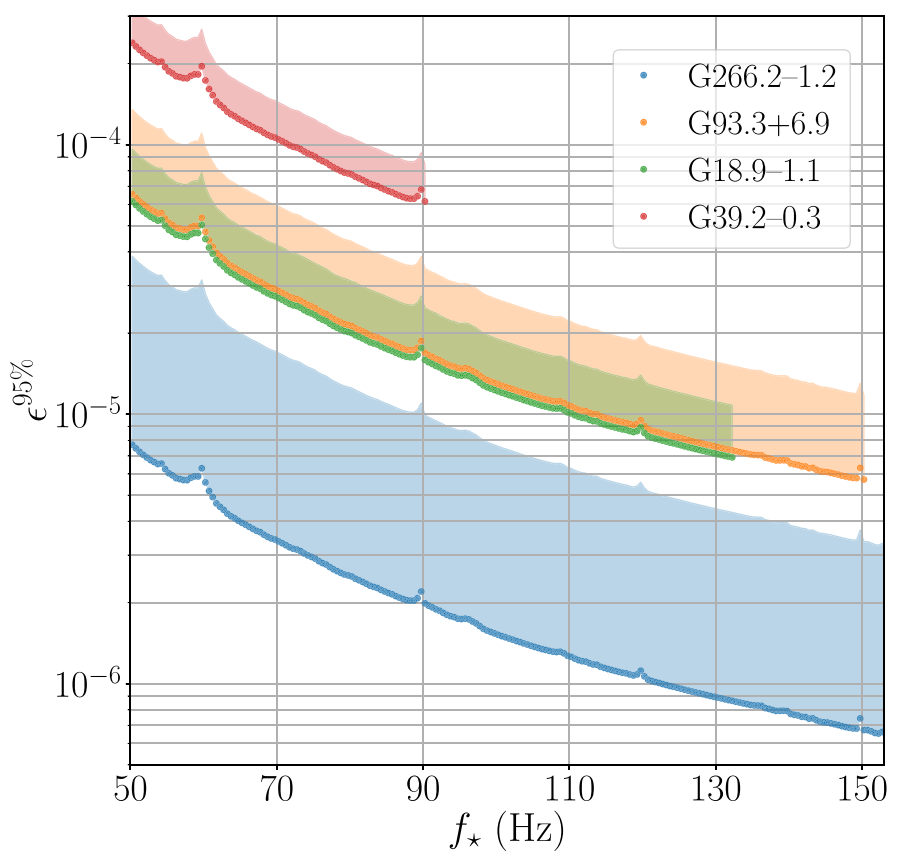

(b)

Figure 6. Constraints on the neutron star ellipticity with $95 \%$ confidence from the dual-harmonic Viterbi search as a function of $f_{\star}$ for (a) $T_{\text {coh }}=12 \mathrm{hr}$ and (b) $T_{\text {coh }}=9 \mathrm{hr}$, converted from the $h_{0}^{95 \%}$ values in Figure 3. The shaded region indicates the resulting ellipticity range calculated from the full estimated distance range for each SNR (see Table 1), and the filled circles correspond to the minimum distance estimate.

Figures 4 and 5 present the constraints on $\epsilon$ and $\alpha$ obtained from the BSD and single-harmonic Viterbi pipelines, respectively. The most stringent constraints, $\epsilon \lesssim 10^{-7}$ and $\alpha \lesssim 10^{-5}$, come from the BSD pipeline (see Figure 4), where the values are converted using the $h_{0}^{95 \%}$ curve from the L detector. The results from the single-harmonic Viterbi pipeline, covering more targets and a wider parameter space, are presented in Figure 5.

We also convert the $h_{0}^{95 \%}$ obtained in the dual-harmonic Viterbi pipeline to the $95 \%$ confidence constraint on the ellipticity of the star. Since GW emission is at both $f_{\star}$ and $2 f_{\star}$, Equation (14) can be written in terms of the spin frequency of the $\operatorname{star} f_{\star}$,

$$
\epsilon=2.36 \times 10^{-5}\left(\frac{h_{0}}{10^{-24}}\right)\left(\frac{D}{1 \mathrm{kpc}}\right)\left(\frac{100 \mathrm{~Hz}}{f_{\star}}\right)^{2} .
$$

Figure 6 shows the limits on $\epsilon$ as a function of $f_{\star}$. Note that the results here are converted from the $h_{0}^{95 \%}$ values obtained for a specific scenario with source properties $\theta=45^{\circ}$ and $\cos \iota=0$, and hence the $\epsilon$ values in Figure 6 are not directly comparable to the results obtained in other conventional searches where $\theta=90^{\circ}$ and the emission is only at $2 f_{\star}$. The signal model adopted in the dual-harmonic search cannot be interpreted as current quadrupole emission from an $r$-mode, so we do not infer $r$-mode amplitudes from $h_{0}^{95 \%}$.

The strictest constraints on the intrinsic GW strain from the BSD pipeline are $h_{0}^{95 \%} \approx 7.7 \times 10^{-26}$ for G39.2-0.3 and $h_{0}^{95 \%} \approx 7.8 \times 10^{-26}$ for $\mathrm{G} 65.7+1.2$ near $200 \mathrm{~Hz}$. The results obtained by the Viterbi pipelines set the first constraints on $\mathrm{CWs}$ that allow for spin wandering in the signal model. Note that the authors of Millhouse et al. (2020) conducted a search for 13 out of the 15 sources in Advanced LIGO O2 data using the single-harmonic Viterbi method but did not derive the constraints from the search sensitivity. Furthermore, the dualharmonic Viterbi analysis provides the first results for these SNR sources derived considering two frequency harmonics simultaneously. The best constraints on the star's ellipticity obtained at frequencies $f \gtrsim 100 \mathrm{~Hz}$ reach $\epsilon<10^{-6}$ for most of the sources, reaching below the rough theoretical upper limit for normal neutron stars (Johnson-McDaniel \& Owen 2013), and reach as low as $\epsilon \approx 6 \times 10^{-8}$ for the closest source G266.2-1.2/Vela Jr., well below the theoretical limits. However, these limits are model dependent; the uncertainties on the star's geometry and composition, like the internal equation of state, the moment of inertia, and the magnetic field, play a significant role when deriving these limits. For example, Woan et al. (2018) show that an ellipticity of $\epsilon \sim 10^{-9}$ can be sustained by neutron stars with a buried magnetic field of $\sim 10^{11} \mathrm{G}$. The most stringent constraints on the $r$-mode amplitude obtained above $\sim 100 \mathrm{~Hz}$ arrive at the theoretical prediction level of $\alpha \sim 10^{-3}$, expected for the nonlinear saturation mechanisms (Bondarescu et al. 2009), and reach as low as $\alpha \sim 10^{-5}$ at higher frequencies.

\section{Conclusion}

In this work, we present the results of the search for CWs from neutron stars in 15 young SNRs by analyzing the data collected in the first half of O3. No evidence of CWs is identified. We present constraints on the GW strain, as well as the implied mass ellipticity and $r$-mode amplitude for each source. The inferred upper limits on the latter quantities reach below the maximum values allowed on theoretical grounds. The strictest constraints on the intrinsic GW strain from the BSD pipeline are $h_{0}^{95 \%} \approx$ $7.7 \times 10^{-26}$ for G39.2-0.3 and $h_{0}^{95 \%} \approx 7.8 \times 10^{-26}$ for G65.7 +1.2 , both near $200 \mathrm{~Hz}$. The Viterbi pipelines set the first constraints on the signal strain allowing for spin wandering. The dual-harmonic Viterbi analysis reports the first results for these 
SNR sources derived considering two frequency harmonics simultaneously. All of the three pipelines are computationally efficient, costing $\sim 10^{2}$ core-hr for each source per pipeline (postprocessing excluded). The significantly improved search efficiency compared to the existing studies for this type of source mainly comes from two factors: (1) we use a much shorter coherence time $T_{\text {coh }}$ in this analysis compared to other studies (e.g., Abbott et al. 2019e; Lindblom \& Owen 2020; Papa et al. 2020), and the computing cost scales roughly as $T_{\text {coh }}^{4}$ to $T_{\text {coh }}^{7}$ depending on the orders of the time derivatives of frequency searched; and (2) advanced signal processing techniques are used in these three pipelines to further accelerate the search, e.g., the dynamic programming in Viterbi pipelines (Viterbi 1967; Sun et al. 2018) and the use of subsampled data and heterodyne correction in the BSD pipeline (Piccinni et al. 2018).

We briefly compare the constraints derived in this work to existing constraints in the literature. The constraints from the two Viterbi-based pipelines are not directly comparable with other analyses because they assume different signal models. The dual-harmonic Viterbi pipeline searches simultaneously for emission at $f_{\star}$ and $2 f_{\star}$. In addition, both Viterbi pipelines search a set of frequency random walks, unlike other pipelines that search a set of Taylor expansion coefficients. A previous search for these sources in $\mathrm{O} 1$ reported $h_{0}^{95 \%} \approx 2 \times 10^{-25}$ for most of the sources and $h_{0}^{95 \%} \approx 1 \times 10^{-25}$ for one source in the most sensitive band (Abbott et al. 2019e), excluding results on Fomalhaut B (Jones \& Sun 2021). The best limit on $h_{0}$ for G39.2-0.3 is $h_{0}^{95 \%} \approx 2 \times 10^{-25}$, approximately 2.5 times higher than the results obtained here for the same source with the BSD pipeline. Lindblom \& Owen (2020) presented a similar search to Abbott et al. (2019e) using the O2 data and reported strain limits slightly above $1 \times 10^{-25}$ at $90 \%$ confidence level, e.g., for G39.2-0.3, which is $\sim 1.4$ times higher than the results obtained here for the same source with the BSD pipeline. Also, using the LIGO data in the full $\mathrm{O} 1$ run and a coherent integration duration longer than 10 days, Papa et al. (2020) set $90 \%$ confidence limits of $h_{0}^{90 \%}=1.2 \times 10^{-25}$, $9.3 \times 10^{-26}$, and $8.8 \times 10^{-26}$ for Cas A, Vela Jr., and G347.3-0.5 near $172.5 \mathrm{~Hz}$, respectively. These limits for Cas A and G347.3-0.5 are lower than those obtained in the singleharmonic Viterbi search for these two SNRs, without any adjustments being made for the different signal models assumed. We do not search for these two SNRs using the BSD and dual-harmonic Viterbi pipelines. The results on Vela Jr. in this analysis from the BSD search slightly improve the previous constraints set by Papa et al. (2020), despite the use of a coherent integration duration $\sim 20$ times shorter. Future data collection and improved analysis methods will further extend the sensitivity of CW searches and increase the probability of a future discovery.

This material is based on work supported by NSF's LIGO Laboratory, which is a major facility fully funded by the National Science Foundation. The authors also gratefully acknowledge the support of the Science and Technology Facilities Council (STFC) of the United Kingdom, the Max-Planck-Society (MPS), and the State of Niedersachsen/Germany for support of the construction of Advanced LIGO and construction and operation of the GEO600 detector. Additional support for Advanced LIGO was provided by the Australian Research Council. The authors gratefully acknowledge the Italian Istituto Nazionale di Fisica Nucleare (INFN), the
French Centre National de la Recherche Scientifique (CNRS), and the Netherlands Organization for Scientific Research, for the construction and operation of the Virgo detector and the creation and support of the EGO consortium. The authors also gratefully acknowledge research support from these agencies, as well as by the Council of Scientific and Industrial Research of India, the Department of Science and Technology, India, the Science \& Engineering Research Board (SERB), India, the Ministry of Human Resource Development, India, the Spanish Agencia Estatal de Investigación, the Vicepresidència i Conselleria d'Innovació Recerca i Turisme and the Conselleria d'Educació i Universitat del Govern de les Illes Balears, the Conselleria d'Innovació Universitats, Ciència i Societat Digital de la Generalitat Valenciana and the CERCA Programme Generalitat de Catalunya, Spain, the National Science Centre of Poland and the Foundation for Polish Science (FNP), the Swiss National Science Foundation (SNSF), the Russian Foundation for Basic Research, the Russian Science Foundation, the European Commission, the European Regional Development Funds (ERDF), the Royal Society, the Scottish Funding Council, the Scottish Universities Physics Alliance, the Hungarian Scientific Research Fund (OTKA), the French Lyon Institute of Origins (LIO), the Belgian Fonds de la Recherche Scientifique (FRS-FNRS), Actions de Recherche Concertes (ARC) and Fonds Wetenschappelijk Onderzoek Vlaanderen (FWO), Belgium, the Paris Île-de-France Region, the National Research, Development and Innovation Office Hungary (NKFIH), the National Research Foundation of Korea, the Natural Science and Engineering Research Council Canada, Canadian Foundation for Innovation (CFI), the Brazilian Ministry of Science, Technology, and Innovations, the International Center for Theoretical Physics South American Institute for Fundamental Research (ICTPSAIFR), the Research Grants Council of Hong Kong, the National Natural Science Foundation of China (NSFC), the Leverhulme Trust, the Research Corporation, the Ministry of Science and Technology (MOST), Taiwan, the United States Department of Energy, and the Kavli Foundation. The authors gratefully acknowledge the support of the NSF, STFC, INFN, and CNRS for provision of computational resources.

This work was supported by MEXT, JSPS Leading-edge Research Infrastructure Program, JSPS Grant-in-Aid for Specially Promoted Research 26000005, JSPS Grant-in-Aid for Scientific Research on Innovative Areas 2905: JP17H06358, JP17H06361, and JP17H06364, JSPS Core-toCore Program A. Advanced Research Networks, JSPS Grantin-Aid for Scientific Research (S) 17H06133, the joint research program of the Institute for Cosmic Ray Research, University of Tokyo, National Research Foundation (NRF) and Computing Infrastructure Project of KISTI-GSDC in Korea, Academia Sinica (AS), AS Grid Center (ASGC) and the Ministry of Science and Technology (MoST) in Taiwan under grants including AS-CDA-105-M06, Advanced Technology Center (ATC) of NAOJ, and Mechanical Engineering Center of KEK.

Note added in proofs. G1.9+0.3 has recently been reclassified as a Type 1a supernova remnant (Borkowski et al. 2017) and should not have been included in this search. We would like to thank Ben Owen and Katie Auchettl for independently informing us of this.

We would like to thank all of the essential workers who put their health at risk during the COVID-19 pandemic, without whom we would not have been able to complete this work. 


\section{Appendix A Candidate Follow-up}

Narrowband noise features and the non-Gaussianity in the interferometric data can cause outliers with detection statistic above the threshold. Consequently, each pipeline requires postprocessing of the results to eliminate candidates originating from noise artifacts. We follow up all the first-stage candidates identified in each pipeline with a hierarchy of predefined veto procedures, as well as additional manual scrutiny. No candidate survives from any pipeline. In this section, we detail the postprocessing procedures.

\section{A.1. Vetoes}

We first describe the predefined veto procedures in this section.

\section{A.1.1. Known-line Veto}

Candidates caused by known instrumental lines are rejected in the first step in all three pipelines. For each candidate identified at a starting frequency $f_{0}$ at $t=0$, we veto the candidate if the band $\left[f_{0}-\delta f, f_{0}+\delta f\right]$ intersects any known instrumental lines present in either the Hanford or Livingston interferometer, where $\delta f=10^{-4} f_{0}$ is used to account for the Doppler shift due to Earth's motion. Note that there is a subtle difference between the pipelines when applying the Dopplershift effect. The BSD and Viterbi pipelines apply $\delta f$ to the line frequency and the candidate frequency, respectively. The three pipelines use a list of known instrumental lines in C01 data (Goetz et al. 2021). In the dual-harmonic Viterbi pipeline, candidates caused by instrumental lines in either of the two separate subbands, corresponding to the $f_{\star}$ and $2 f_{\star}$ components, are rejected.

\section{A.1.2. Interferometer Veto}

In general, a candidate signal with an astrophysical origin should be present in the data of all detectors. If the candidate is louder in one detector than the other, it ought to be louder in the detector with better sensitivity for the source and in the frequency band considered. Each pipeline therefore applies a veto to the consistency of the candidate signal strength across each detector.

For the BSD algorithm, this means vetoing candidates with a weighted $\mathrm{CR}$ in the less sensitive detector more than three times higher than the corresponding weighted $\mathrm{CR}$ in the more sensitive one. This is applied using the CR computed from the statistical distribution of the FH number counts. This veto is repeated after the next veto step A.1.3, using another statistic, namely, the 5-vector statistic (Astone et al. 2010, 2014a), as the second round of consistency check. All the candidates with $\rho_{\mathrm{CR}_{1}} / \sqrt{S_{n_{1}}}>3 \rho_{\mathrm{CR}_{2}} / \sqrt{S_{n_{2}}}$ are vetoed, where $\sqrt{S_{n_{i}}}$ is the noise amplitude spectral density in each $i$ th detector, and assuming that detector 1 is less sensitive than detector 2 . The factor of 3 is a conservative choice (recently used in Abbott et al. (2019f)).

For the Viterbi algorithms, we repeat the full search over $T_{\text {obs }}$ in each individual interferometer. The candidate is vetoed if the two criteria are both satisfied: (a) searching data from a single interferometer yields $\mathcal{L} \geqslant \mathcal{L} \cup$ in single-harmonic Viterbi $\left(S \geqslant S_{\cup}\right.$ in dual-harmonic Viterbi), where $\mathcal{L}_{\cup}\left(S_{\cup}\right)$ is the original statistic obtained with both interferometers combined, while searching the other interferometer yields $\mathcal{L}<\mathcal{L} \cup$
$\left(S<S_{\text {th }}\right)$; and (b) the Viterbi path from the interferometer with $\mathcal{L} \geqslant \mathcal{L}_{\cup}\left(S \geqslant S_{\cup}\right)$ intersects the original path, i.e., the increased significance in a single detector occurs at the same frequency as the original candidate.

\section{A.1.3. Doppler-shift (and Spin-down-shift) Veto}

All three pipelines apply a Doppler correction to transform the observation in the detector frame to the source frame. For a true astrophysical signal, this correction should increase the significance of any candidate in the data; for local noise, the significance should decrease or remain unaffected. Both the BSD and the dual-harmonic Viterbi pipeline apply a veto based on this correction. This is not applied to the single-harmonic Viterbi pipeline because the $T_{\text {coh }}$ are short enough to track the Doppler shift.

For the BSD algorithm, we compute the significance of the candidate in terms of the $\mathrm{CR}$ and signal-to-noise ratio ( $\rho_{i}$ where $i=\mathrm{CR}$, snr) with and without the Doppler and spin-down corrections applied to the time series. The Doppler and spindown corrections are done using a heterodyne phase correction, where the assumed phase evolution of the signal, $\phi\left(f_{0}, \dot{f}_{0}\right)$, is fully described by the frequency and spin-down parameters of the candidate, given by $f_{0}$ and $\dot{f}_{0}$, respectively. The corrected time series is computed by multiplying the original (uncorrected) time series by the exponential factor $\exp j \phi\left(f_{0}, \dot{f}_{0}\right)$. An easy way to compute the statistical significance of a candidate, once the $\phi\left(f_{0}, \dot{f}_{0}\right)$ is assumed to be known, is to use the 5-vector statistics (Astone et al. 2010, 2014a), originally developed for the search of known pulsars. Hence, for this step, we use a statistic based on the 5-vector method, whose main properties are described in Appendix B.1.2. We use $\rho_{\mathrm{CR}}$ and $\rho_{\text {snr }}$ to check the nature of a candidate, but this time we compute them from the 5-vector statistic $\mathcal{S}$ rather than from the FH number count. For this reason, the CR computed in this step is not directly comparable with the CR of the FH map used for the first-level selection of candidates. If a candidate is from astrophysical origin, we expect, after testing the procedure with simulated signals injected in $\mathrm{O} 3$ data, that the significance will increase after the correction, and that it would increase proportionally to the fourth root of the coherence time. This comparison is done using two different coherence times, $T_{\text {sid }}$ and $T_{4 \text { sid }}=4 T_{\text {sid }}$, where $T_{\text {sid }}=86,164.0905 \mathrm{~s}$ is the duration of a sidereal day. We use the 5-vector $\rho_{i} \mathrm{CR}$ and signal-to-noise ratio to check the change of significance. We keep the candidates if a larger 5-vector $\rho_{\mathrm{CR}, \mathrm{C}}$ is obtained with the correction applied than the 5 -vector $\rho_{\mathrm{CR}, \mathrm{NC}}$ obtained without the correction applied (in the two cases using $T_{\text {sid }}$ and $T_{4}$ sid). We also veto those candidates that do not show an increased signal-to-noise ratio after the correction. Simulation studies show that the false dismissal probability of this veto is below $10 \%$ if a tolerance of $5 \%$ is used, e.g., we keep all those candidates with $\rho_{i, C}-0.95 \rho_{i, \mathrm{NC}}>0$, where $\rho_{i, \mathrm{C}}$ and $\rho_{i, \mathrm{NC}}$ refer to the corrected and uncorrected case, respectively.

In the dual-harmonic Viterbi search, for each candidate remaining, we recompute $\mathcal{F}$-statistics over the same $T_{\text {coh }}$ as listed in Table 4 with Doppler modulation correction turned off (DM-off) and repeat the search using the DM-off $\mathcal{F}$-statistics (Zhu et al. 2017). If the candidate is of astrophysical origin, it should become undetectable in the DM-off search, returning a score $S_{\mathrm{DM}-\text { off }}<S_{\mathrm{th}}$ and a Viterbi path different from the original one. This criterion does not apply to a high signal-tonoise ratio candidate, i.e., $S \gg S_{\text {th }}$. However, after previous 
veto steps, no such high signal-to-noise ratio candidate is left in this search. Instead, if a candidate is caused by noise artifacts on Earth, its significance is expected to increase in the followup. Hence, we veto a candidate if the DM-off follow-up yields $S_{\mathrm{DM}-\text { off }} \geqslant S_{\cup}$ and returns a new Viterbi path intersecting the band $\left[f_{0}-\delta f, f_{0}+\delta f\right]$.

\section{A.1.4. Sky-position Veto}

If a candidate is of astrophysical origin, it should yield the highest detection statistic at the sky position of the source (Isi et al. 2020). For candidates surviving previous steps, the Viterbi pipelines conduct another follow-up step by shifting the sky position away from the true position of the SNR (Jones \& Sun 2021). This off-target veto contains two separate parts: (a) shift R.A. by an offset $\delta_{\mathrm{RA}}$ while keeping decl. fixed at the true location, and (b) shift decl. by $\delta_{\mathrm{DEC}}$ while keeping R.A. fixed at the true location. We use $\delta_{\mathrm{RA}}=3 \mathrm{hr}$ and $\delta_{\mathrm{DEC}}=30^{\circ}$. These offset values are chosen based on a large number of Monte Carlo simulations that pass the veto safety check. For the sources with a decl. angle in the range of $\left[-90^{\circ}, 0^{\circ}\right]$ and $\left(0^{\circ}, 90^{\circ}\right]$, we set $\delta_{\mathrm{DEC}}$ to $30^{\circ}$ and $-30^{\circ}$, respectively. The single-harmonic Viterbi pipeline conducts (a) only. The dual-harmonic Viterbi pipeline conducts both (a) and (b). For (a), we veto the candidate if the off-target search yields $\mathcal{L}_{\text {off-target }}>\mathcal{L}_{\text {th }}\left(S_{\text {off-target }}>S_{\cup}\right)$ and returns a new Viterbi path intersecting the band $\left[f_{0}-\delta f, f_{0}+\delta f\right]$. For (b), we veto the candidate if the off-target search yields $S_{\text {off-target }}>S_{\text {th }}$ and returns a new Viterbi path intersecting the band $\left[f_{0}-\delta f, f_{0}+\delta f\right]$. Note that the veto criterion for (b) is more stringent than that for (a) in the dual-harmonic Viterbi pipeline, because this analysis is more sensitive to the mismatch along the direction of decl. Only the candidates surviving both (a) and (b) are kept.

\section{A.1.5. Cumulative-significance Veto}

The significance of a CW signal should be consistent over $T_{\text {obs }}$, and in the presence of stationary noise, there should be no sudden increase or decrease in the significance when more data are used to integrate the signal. The BSD algorithm uses the 5-vector statistics (Astone et al. 2010, 2014a) to compute the cumulative signal-to-noise ratio and $\mathrm{CR}$ on a monthly basis, increasing the amount of data used in each iteration by 1 month. We also compute this trend using a heterodynecorrected time series with a phase correction $\phi\left(f_{0}, \dot{f}_{0}\right)$ obtained from the candidate's parameters $\left(f_{0}, \dot{f}_{0}\right)$. The two trends, derived from the corrected and uncorrected time series, are then compared. The comparison is done looking at the plots of the $\mathrm{CR}$, the signal-to-noise ratio, and the 5-vector statistics $\mathcal{S}$ (defined in Equation (B7) of Appendix B.1.2) as a function of the number of months used to compute these quantities. We visually inspect these plots by comparing the trend of both curves in the corrected and uncorrected case. We veto the candidates if the plot of the corrected case has lower values than the corresponding uncorrected case for the entire duration of the run. We also veto candidates when the CR cumulative curve of the most sensitive detector is well below the less sensitive one, which is a clear clue that the candidate is actually due to some noise present in the less sensitive detector. For other, more complicated cases, we do not automatically veto the candidate but leave them for further investigation in the full $\mathrm{O} 3 \mathrm{H}$ and $\mathrm{L}$ data. This is a conservative choice, since vetoing all the candidates that simply present a sudden increase or decrease in the significance (in terms of CR, signal-to-noise ratio, and 5-vector statistic value) is not safe when the noise is not Gaussian.

\section{A.1.6. Subband Veto}

If a subband is heavily contaminated by non-Gaussian noise, it can be challenging to distinguish noise from a candidate signal. In the case of the single-harmonic Viterbi pipeline, this renders $\mathcal{L}_{\text {th }}$ invalid because $\mathcal{L}_{\text {th }}$ is calculated using the results of Gaussian noise simulations. Furthermore, we do not expect multiple CW signals in a single subband. Consequently, we veto any candidates in a subband if the subband has more than two unique Viterbi paths with $\mathcal{L}>\mathcal{L}_{\text {th }}$. Simulations in Gaussian noise found that $<1 \%$ of bands returned two unique paths with $\mathcal{L}>\mathcal{L}_{\text {th }}$, justifying our assumption that a subband with multiple candidates is dominated by non-Gaussian noise. The dual-harmonic Viterbi pipeline uses the Viterbi score as the detection statistic and only keeps the optimal path, and hence this step does not apply.

\section{A.1.7. Coherence-time Veto}

Both implementations of the Viterbi algorithm use the $\mathcal{F}$-statistic computed over each $T_{\text {coh }}$ interval in Tables 3 and 4. In the original search, we select $T_{\text {coh }}$ assuming a range of $\dot{f}_{0}$. Candidates returned with relatively low $\left|\dot{f}_{0}\right|$ allow us to increase the coherent time in a follow-up search. The sensitivity of the $\mathcal{F}$-statistic increases with longer $T_{\text {coh }}$, as long as there is no power leakage over $T_{\text {coh }}$ given the inferred $\left|\dot{f}_{0}\right|$; a more sensitive $\mathcal{F}$-statistic facilitates a more sensitive Viterbi search. Hence, the significance of the candidate should increase with longer $T_{\text {coh }}$ if the candidate is a real astrophysical signal. This has been verified using simulations.

In practice, we first calculate the mean $\dot{f}_{0}$ value over the candidate path and then estimate the maximum $T_{\text {coh }}$ capable of tracking the inferred spin-down. In the single-harmonic Viterbi pipeline, we conduct a follow-up search using $T_{\text {coh }}=4 \mathrm{hr}$ for all survivors. With the increased $T_{\text {coh }}$, the ratio $\mathcal{L} / \mathcal{L}_{\text {th }}$ should increase for a real signal, so we veto any candidates for which $\mathcal{L}\left(T_{\text {coh }}=4 \mathrm{hr}\right) / \mathcal{L}_{\text {th }}\left(T_{\text {coh }}=4 \mathrm{hr}\right)<\mathcal{L} / \mathcal{L}_{\text {th }}$, where $\mathcal{L} / \mathcal{L}_{\text {th }}$ are the values from the initial search. Similarly, in the dualharmonic Viterbi pipeline, we veto a candidate if a decreased Viterbi score is returned with the increased $T_{\text {coh }}$. No candidate survives in the dual-harmonic Viterbi pipeline after this step.

\section{A.2. Further Verification}

After the hierarchy of well-defined veto steps, we discuss the additional verification conducted in each pipeline.

\section{A.2.1. BSD Follow-up}

A total of 35 candidates identified by the BSD pipeline survive the vetoes described in Appendix A.1. This is consistent given the low CR threshold chosen in Section 4.1; indeed, we are exposed to false-alarm candidates, and most of them could arise from noise fluctuations. The $\rho_{\mathrm{CR}, \mathrm{thr}}$ chosen corresponds to the probability of picking, on average, more than one noise candidate. Indeed, the CR threshold corresponding to the selection of only one false candidate over the total number of points in the parameter space would be $\rho_{\mathrm{CR} \text {,thr }} \sim 5.7$, while, for instance, in the search described in Piccinni et al. (2020) the CR threshold used is 6.5. In this section, we describe the extra steps taken to investigate and 
Table 5

Surviving Candidates from the BSD Pipeline after Vetos in Appendix A.1

\begin{tabular}{|c|c|c|c|}
\hline Source & Original CR $(\mathrm{FH})$ & $f_{0}(\mathrm{~Hz})$ & $\dot{f}_{0}\left(\mathrm{~Hz} \mathrm{~s}^{-1}\right)$ \\
\hline \multirow[t]{6}{*}{$\mathrm{G} 189.1+3.0$} & 5.40 & 65.6458598 & $-3.6202 \times 10^{-10}$ \\
\hline & 4.80 & 75.2342077 & $-7.9666 \times 10^{-10}$ \\
\hline & 5.44 & 117.6621941 & $-7.3779 \times 10^{-10}$ \\
\hline & 4.89 & 321.8914027 & $-3.3898 \times 10^{-9}$ \\
\hline & 4.96 & 355.9654409 & $-1.1674 \times 10^{-9}$ \\
\hline & 4.90 & 498.1299761 & $-1.9622 \times 10^{-9}$ \\
\hline \multirow[t]{2}{*}{$\mathrm{G} 65.7+1.2$} & 5.56 & 97.5785468 & $-8.5222 \times 10^{-10}$ \\
\hline & 4.92 & 120.7226480 & $-1.0230 \times 10^{-9}$ \\
\hline \multirow[t]{6}{*}{ G266.2-1.2 } & 5.07 & 90.3241483 & $4.0128 \times 10^{-11}$ \\
\hline & 5.48 & 101.8200802 & $-6.1815 \times 10^{-10}$ \\
\hline & 5.18 & 139.6310303 & $-1.1902 \times 10^{-10}$ \\
\hline & 5.07 & 142.8760455 & $-2.7103 \times 10^{-12}$ \\
\hline & 4.87 & 217.9480484 & $2.1858 \times 10^{-10}$ \\
\hline & 5.15 & 274.5506302 & $1.1958 \times 10^{-10}$ \\
\hline \multirow[t]{5}{*}{ G93.3+6.9 } & 5.61 & 208.8871413 & $-9.6487 \times 10^{-10}$ \\
\hline & 5.25 & 758.8185308 & $-4.4728 \times 10^{-9}$ \\
\hline & 4.83 & 807.0006637 & $-2.6116 \times 10^{-9}$ \\
\hline & 4.86 & 851.3276865 & $-3.9519 \times 10^{-9}$ \\
\hline & 5.49 & 858.9144800 & $-3.9696 \times 10^{-9}$ \\
\hline \multirow[t]{6}{*}{ G18.9-1.1 } & 5.24 & 65.0196186 & $-4.7373 \times 10^{-11}$ \\
\hline & 5.18 & 91.4561088 & $-4.2390 \times 10^{-10}$ \\
\hline & 5.78 & 332.4543731 & $-3.6398 \times 10^{-10}$ \\
\hline & 4.88 & 488.0994499 & $-2.5631 \times 10^{-9}$ \\
\hline & 4.72 & 841.6011602 & $-2.8949 \times 10^{-9}$ \\
\hline & 4.95 & 844.8167574 & $-4.1613 \times 10^{-9}$ \\
\hline \multirow[t]{8}{*}{ G39.2-0.3 } & 4.98 & 135.6997524 & $-7.5073 \times 10^{-10}$ \\
\hline & 4.77 & 333.8910938 & $-2.1219 \times 10^{-9}$ \\
\hline & 4.76 & 334.0656179 & $-1.7914 \times 10^{-9}$ \\
\hline & 4.76 & 700.7408355 & $-3.9014 \times 10^{-9}$ \\
\hline & 5.12 & 721.1474692 & $-2.9249 \times 10^{-9}$ \\
\hline & 5.35 & 802.0985150 & $-3.0294 \times 10^{-9}$ \\
\hline & 4.68 & 831.8034158 & $-6.6128 \times 10^{-10}$ \\
\hline & 5.31 & 902.3896162 & $-4.9226 \times 10^{-9}$ \\
\hline \multirow[t]{2}{*}{ G353.6-0.7 } & 5.36 & 64.6267590 & $-9.1944 \times 10^{-12}$ \\
\hline & 4.99 & 754.3285519 & $-4.0769 \times 10^{-10}$ \\
\hline
\end{tabular}

Note. The candidates investigated were excluded using the full $\mathrm{O} 3$ data in the BSD search. The columns list the source name, the original mean CR from the FH map, the candidate frequency $f_{0}$, and the spin-down $\dot{f}_{0}$ at the time of the coincidences. The initial set of candidates has been selected using $\rho_{\mathrm{CR} \text {,thr }}=4.7$ for G65.7+1.2, G189.1+3.0, and G266.2-1.2; $\rho_{\mathrm{CR}, \mathrm{thr}}=4.6$ for G18.9-1.1 and $\mathrm{G} 93.3+6.9$; and $\rho_{\mathrm{CR}, \mathrm{thr}}=4.5$ for G353.6-0.7 and G39.2-0.3.

disqualify the surviving candidates (listed in Table 5). We repeat the Doppler-shift (and spin-down-shift) veto (Appendix A.1.3), the cumulative-significance veto (Appendix A.1.5), and the interferometer veto (Appendix A.1.2) using the full O3 (O3a and $\mathrm{O} 3 \mathrm{~b}$ ) $\mathrm{C} 01$ data. We remind the reader that the $\mathrm{CR}$ computed by the $\mathrm{FH}$ is based on the number count associated with the pixel in the FH map where the candidate has been found, while the CR in the 5-vector is computed from the $\mathcal{S}$ statistic. In this step, we use the CR from the $\mathcal{S}$ statistic. None of the candidates in Table 5 survive the full $\mathrm{O} 3$ vetos, and as can be seen, the original $\mathrm{CR}$ associated with the candidate in the FH map is below the $\rho_{\mathrm{CR} \text {,thr }} \sim 5.7$, hence with a very low significance compatible with noise.

\section{A.2.2. Single-harmonic Viterbi Follow-up}

One candidate identified by the single-harmonic Viterbi pipeline survives the veto process defined in Appendix A.1. The candidate

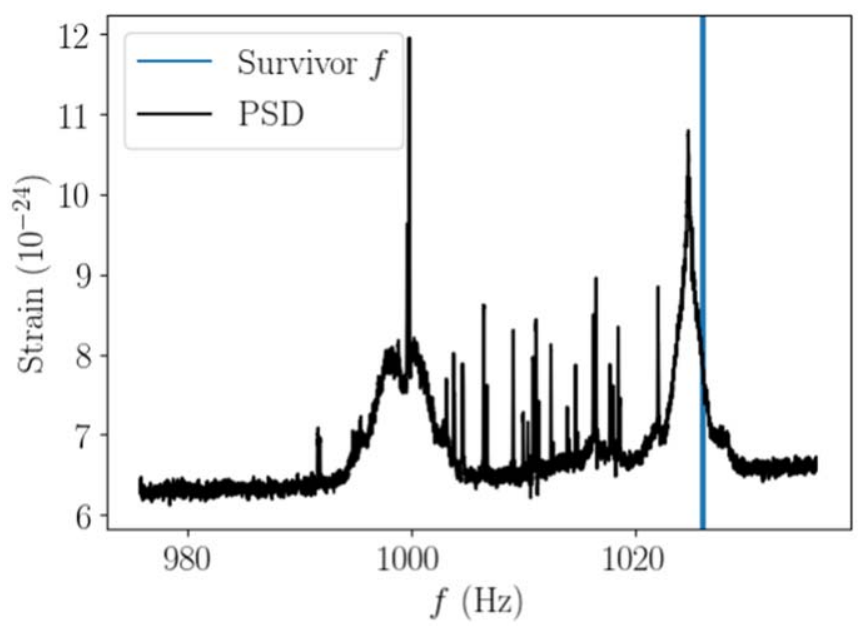

Figure 7. Power spectral density (black curve) vs. frequency and the frequency of the last surviving candidate for source G93.3+6.9 (blue vertical line). The power spectral density is built using data collected from both Hanford and Livingston detecters over the full observing time of O3a.

is associated with G93.3+6.9 and has a frequency path with mean $f_{0}=1025.95 \mathrm{~Hz}$ and $\dot{f}_{0}=-2.13 \times 10^{-9} \mathrm{~Hz} \mathrm{~s}^{-1}$ and a likelihood $\mathcal{L}=18154.0$, within $5 \%$ of $\mathcal{L}_{\text {th }}$. In Figure 7 , we plot the power spectral density (black curve) for both detectors combined, calculated over the full duration of $\mathrm{O} 3 \mathrm{a}$, and overplot the frequency of the surviving path (blue vertical line). Visual inspection plainly indicates that it is associated with noise. Though it does not lie on the peak of the noise disturbance, it is in the wings.

\section{A.2.3. Dual-harmonic Viterbi Cross-check}

All candidates identified in this search pipeline are rejected after the veto procedure described in Appendix A.1.

Here we provide more details of the last set of candidates processed in the coherence-time veto (Appendix A.1.7). In Table 6 , the original Viterbi score, estimated $2 f_{\star}$ at the beginning and the end of the observation $\left(2 f_{\star \text { start }}\right.$ and $\left.2 f_{\star \text { end }}\right)$, and the mean $2 \dot{f}_{\star}$ values (i.e., $\left.\left(2 f_{\star \text { start }}-2 f_{\star \text { end }}\right) / T_{\text {obs }}\right)$ of each candidate are provided. Note that the pipeline returns estimated frequencies and spin-down rates corresponding to the $2 f_{\star}$ component. We directly report the returned values in this section rather than converting them into the $f_{\star}$ component. The increased $T_{\text {coh }}$ used for each candidate is listed in the sixth column. All of the new scores obtained by increasing $T_{\text {coh }}$ fall below the original score. They are all considered vetoed according to the criteria set in Appendix A.1.7. To be more conservative, we further discuss the only two candidates with new scores above $S_{\text {th }}$ (although decreased compared to the original score), marked by "**" and " $\uparrow$ " in the table. The one marked by "“*” can be confidently ruled out since it returns a completely different path. The follow-up search for the candidate marked by " $\dagger$ " using $T_{\text {coh }}=11 \mathrm{hr}$ yields a lower mean spin-down rate, $2 \dot{f}_{\star}=-1.56 \times 10^{-10} \mathrm{~Hz} \mathrm{~s}^{-1}$, which allows us to further increase $T_{\text {coh }}$. By searching the same subband using $T_{\text {coh }}=15 \mathrm{hr}$, we find that the optimal path overlaps with the original one, but with a further decreased significance, $S=4.97$. Hence, it does not survive the further scrutiny.

Next, we describe additional verification conducted to ensure that we do not veto a weak signal at the final step accidentally. For all the final-stage candidates in Table 6, we cross-check 
Table 6

Final Candidates from the Dual-harmonic Viterbi Pipeline and the Coherence-time Veto Results (All Vetoed)

\begin{tabular}{|c|c|c|c|c|c|c|c|}
\hline Source & $S$ & $2 f_{\star \text { start }}(\mathrm{Hz})$ & $2 f_{\text {*end }}(\mathrm{Hz})$ & Mean $2 \dot{f}_{\star}\left(10^{-10} \mathrm{~Hz} \mathrm{~s}^{-1}\right)$ & New $T_{\text {coh }}(\mathrm{hr})$ & New $S$ & Paths Overlap? \\
\hline \multirow[t]{10}{*}{ G353.6-0.7 } & 5.48 & 129.1071064815320 & 129.1048495370870 & -1.4276 & 15 & 5.17 & $\checkmark$ \\
\hline & 5.68 & 130.0884606481900 & 130.0864004630040 & -1.30314 & 15 & $5.49^{*}$ & $\times$ \\
\hline & 5.52 & 256.5881944432800 & 256.5861342580990 & -1.30314 & 15 & 5.08 & $\checkmark$ \\
\hline & 5.56 & 416.7312615726270 & 416.7296180541120 & -1.03959 & 15 & 4.85 & $\checkmark$ \\
\hline & 5.52 & 453.4695254620340 & 453.4668981472240 & -1.66187 & 15 & 4.45 & $\times$ \\
\hline & 5.80 & 464.3793518511010 & 464.3771180548090 & -1.41296 & 15 & 4.33 & $\times$ \\
\hline & 5.82 & 578.1490972219270 & 578.1467592589690 & -1.47885 & 15 & 4.71 & $\times$ \\
\hline & 5.84 & 636.2839467586970 & 636.2820833327750 & -1.17869 & 15 & 4.26 & $\times$ \\
\hline & 5.60 & 742.4446990731940 & 742.4426273139391 & -1.31046 & 15 & 5.21 & $\times$ \\
\hline & 5.82 & 870.4499074065170 & 870.4476504620770 & -1.4276 & 15 & 3.64 & $\times$ \\
\hline G189.1+3.0 & 5.66 & 253.1534143519250 & 253.1509490741460 & -1.55938 & 15 & 4.34 & $\times$ \\
\hline \multirow[t]{6}{*}{ G65.7+1.2 } & 5.49 & 248.5372569447000 & 248.5349537039590 & -1.45689 & 15 & 4.72 & $x$ \\
\hline & 5.77 & 269.5971180543740 & 269.5952662025260 & -1.17137 & 15 & 4.74 & $\times$ \\
\hline & 5.71 & 277.6064583321330 & 277.6043981469520 & -1.30314 & 15 & 5.12 & $\times$ \\
\hline & 5.69 & 321.1599189811650 & 321.1574537033920 & -1.55938 & 15 & 5.03 & $x$ \\
\hline & 5.74 & 404.2299537032490 & 404.2277314810310 & -1.40564 & 15 & 4.34 & $\times$ \\
\hline & 5.51 & 486.9126851833790 & 486.9104398130130 & -1.42028 & 15 & 3.94 & $x$ \\
\hline \multirow[t]{2}{*}{ G266.2-1.2 } & 5.76 & 172.1456481481280 & 172.1412808641780 & -2.76247 & 11 & 4.96 & $\times$ \\
\hline & 5.45 & 296.5154012345940 & 296.5116358024890 & -2.38178 & 12 & 3.74 & $x$ \\
\hline \multirow[t]{2}{*}{ G93.3+6.9 } & 5.51 & 121.0981018518380 & 121.0943364197400 & -2.38178 & 12 & 4.45 & $\times$ \\
\hline & 6.49 & 138.5975617282440 & 138.5931481479980 & -2.79175 & 11 & $5.80 \dagger$ & $\checkmark$ \\
\hline \multirow[t]{3}{*}{ G18.9-1.1 } & 5.34 & 193.5356481480750 & 193.5317746912850 & -2.45011 & 12 & 4.64 & $\checkmark$ \\
\hline & 5.71 & 219.8922530862280 & 219.8871604936370 & -3.22125 & 12 & 3.68 & $\times$ \\
\hline & 5.85 & 253.5746296295510 & 253.5707407406620 & -2.45987 & 12 & 4.06 & $\times$ \\
\hline G39.2-0.3 & 6.33 & 109.5362962962230 & 109.5325771604210 & -2.35249 & 12 & 4.91 & $x$ \\
\hline
\end{tabular}

Note. The first five columns list the source name, original score, estimated start and end $2 f_{\star}$, and the mean $2 \dot{f}_{\star}$. The sixth column lists the new $T_{\text {coh }}$ used in the coherence-time veto. The last two columns show the follow-up results: the new score obtained by increasing $T_{\text {coh }}$, and whether the new optimal path overlaps the original candidate path. The top and bottom halves of the table correspond to the searches using original $T_{\text {coh }}=12 \mathrm{hr}\left(S_{\text {th }}=5.47\right)$ and $T_{\text {coh }}=9 \mathrm{hr}\left(S_{\text {th }}=5.33\right)$, respectively. The GPS times for the start and end of the observation are 1238166353 and 1253975702 , respectively. The two new scores marked by “*” and " $\dagger$ " are above $S_{\text {th. }}$. (Note that the pipeline returns estimated frequencies and spin-down rates corresponding to the $2 f_{\star}$ component.)

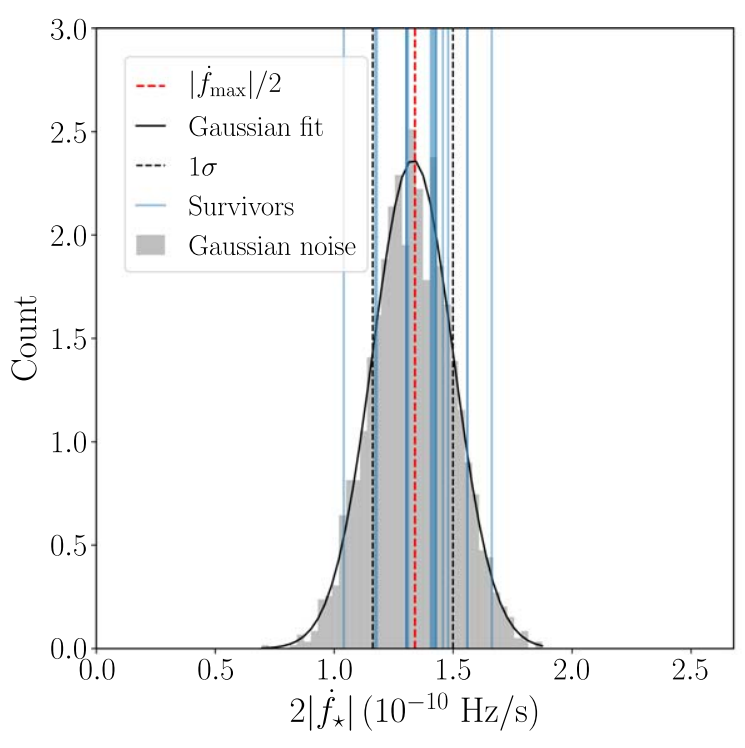

(a)

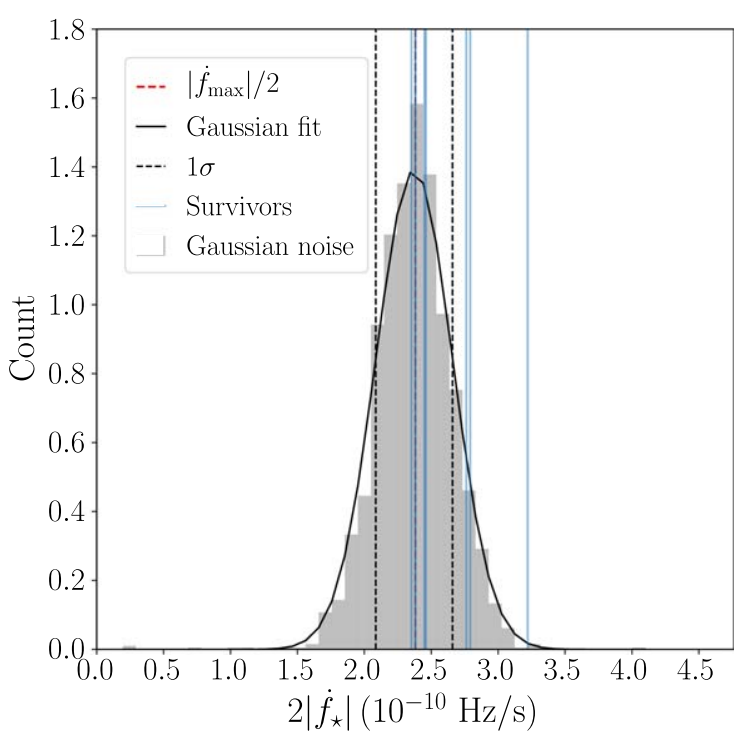

(b)

Figure 8. Noise-only distribution of the mean $2\left|\dot{f}_{\star}\right|$ (gray histogram) and the values obtained from the remaining candidates (blue vertical lines) for (a) $T_{\text {coh }}=12 \mathrm{hr}$ and (b) $T_{\text {coh }}=9 \mathrm{hr}$ in the dual-harmonic Viterbi search. The right edge of each panel is the maximum spin-down rate $\left|\dot{f}_{\max }\right|=1 /\left(2 T_{\text {coh }}^{2}\right)$ for the $2 f_{\star}$ component. The red dashed line indicates $\left|\dot{f}_{\max }\right| / 2$. The noise-only distribution is obtained from 2000 Gaussian noise realizations for each panel. The black solid curve indicates the Gaussian fit of the noise distribution. The two black dashed lines are the $\pm 1 \sigma$ bounds.

them by searching in the data collected over the second half of $\mathrm{O} 3$, with the same configuration as in the original search, in the subbands where these candidates are found. None of the optimal paths returned in O3b data overlap the original path (taking into consideration the possible spin-down during the shutdown time between two halves of observation). 
A further consistency verification is conducted for the candidates in Table 6 . All of them have low scores of $S \lesssim$ $1.2 S_{\text {th }}$ (cf. $S \sim 5 S_{\text {th }}$ vetoed at early steps). Hence, we examine whether they are false alarms arising from noise given that the threshold chosen corresponds to $1 \%$ false-alarm probability. Since the signal frequency is approximated by a negatively biased random walk, the mean $2\left|\dot{f}_{\star}\right|$ value of a path obtained from pure Gaussian noise over $T_{\text {obs }}$ is expected to be around $\left|\dot{f}_{\max }\right| / 2$, where $\left|\dot{f}_{\max }\right|=1 /\left(2 T_{\text {coh }}^{2}\right)$ is the maximum spin-down rate covered in the search for the $2 f_{\star}$ component. This is because the method attempts to "track" pure Gaussian noise with a transition probability $A_{q_{i-1} q_{i}}=A_{q_{i} q_{i}}=1 / 2$ and $2\left|\dot{f}_{\star}\right|$ can take any value in the range of $\left[0,\left|\dot{f}_{\max }\right|\right]$. Figure 8 shows the distribution of the mean $2\left|\dot{f}_{\star}\right|$ obtained by tracking 2000 pure Gaussian noise realizations (gray histograms; fit with black curve) and the values from the remaining candidates (blue vertical lines). The left and right edges of each panel are the minimum and maximum spin-down rates covered in the search, respectively. Out of all 25 candidates, 18 lie within the interval of $[-\sigma, \sigma]$ (black dashed lines). For both $T_{\text {coh }}=12 \mathrm{hr}$ and $T_{\text {coh }}=9 \mathrm{hr}$, all the candidate paths are consistent with pure noise. Moreover, the total number of remaining candidates is consistent with the false-alarm probability $(1 \%$ in each subband). Hence, these candidates with low scores are likely to be false alarms. This explains why they are not confidently rejected at early steps.

\section{Appendix B Details on Search Pipelines}

\section{B.1. BSD}

\section{B.1.1. Impact of the Age and the Second-order Spin-down}

As mentioned in Section 4.1, the age of the source sets the range of frequency and spin-down/up we can investigate for a given target, and it does not directly affect the sensitivity of the pipeline as it happens, e.g., for the coherence time. In the BSD search the first- and second-order spin-down/up (typically referred to as spin-down) ranges are defined by the age $t_{\text {age }}$ and the braking index $n$ of the source as

$$
\left\{\begin{array}{l}
-f / t_{\text {age }} \leqslant \dot{f} \leqslant 0.1 f / t_{\text {age }} \\
0 \mathrm{~Hz} \mathrm{~s}^{-2} \leqslant \ddot{f} \leqslant n|\dot{f}|_{\max }^{2} / f=n f / t_{\text {age }}^{2}
\end{array} .\right.
$$

Since we are not explicitly removing the frequency modulation due to the second-order spin-down, we will limit our search to those sources for which second-order spin-down range is constrained in a single second-order spin-down bin $\delta \ddot{f}$. In practice, we require that $n f / t_{\text {age }}^{2} \leqslant \delta \ddot{f}$. Given that the size of the second-order spin-down bin is $\delta \ddot{f}=\delta f T_{\text {obs }}^{-2}$ (as in Frasca et al. 2005; Astone et al. 2014a), where $\delta f$ is the frequency bin size and proportional to $\sqrt{f}$, we can write the maximum frequency allowed for a given source (in the case of a spin-down dominated by gravitational emission, hence $n=5$ ) as

$$
f \leqslant 4.85 \times 10^{-13}\left(\frac{t_{\text {age }}}{T_{\text {obs }}}\right)^{4} \mathrm{~Hz} .
$$

This means that, e.g., for a source of $t_{\mathrm{age}}=3 \mathrm{kyr}$ and $T_{\text {obs }}$ equal to the $\mathrm{O} 3 \mathrm{a}$ run length, the maximum frequency covered in the search, neglecting the second-order spin-down modulation, is $\sim 600 \mathrm{~Hz}$.

\section{B.1.2. Follow-up Based on the 5-vector Statistic}

In this section, we briefly recap the 5-vector method and its statistic in order to describe the new follow-up veto steps (Appendices A.1.3 and A.1.5) used in this search by the BSD pipeline. The 5-vector detection statistic is built exploiting the feature of the amplitude modulation we observe at the detector. This modulation is induced by the detector radiation pattern in response to a $\mathrm{CW}$ signal and, given that the interferometers are on Earth, also by the change of the received polarization, called sidereal modulation. The response of the detector to a passing CW signal, after removing the Doppler and spin-down frequency modulations, can be described as (see Astone et al. 2010 for more details)

$$
\begin{aligned}
h(t)= & H_{0}(\eta)\left[H_{+}(\psi, \eta) A_{+}(t)\right. \\
& \left.+H_{\times}(\psi, \eta) A_{\times}(t)\right],
\end{aligned}
$$

where $A_{+/ \times}(t)$ are the two sidereal responses to plus and cross polarizations and $H_{0}(\eta)$ is the maximum signal strain. The plus and cross amplitudes $H_{+/ \times}$are given by

$$
\begin{aligned}
& H_{+}=\frac{\cos (2 \psi)-i \eta \sin (2 \psi)}{\sqrt{1+\eta^{2}}}, \\
& H_{\times}=\frac{\sin (2 \psi)-i \eta \cos (2 \psi)}{\sqrt{1+\eta^{2}}},
\end{aligned}
$$

and they depend on the polarization angle $\psi$ and the parameter $\eta$, which denotes the degree of polarization of the $\mathrm{CW}(\eta=0$ for a linearly polarized wave, $\eta= \pm 1$ for a circularly polarized wave). It can be shown (see, e.g., Astone et al. 2010) that the frequency components of the signal at the detector are all encoded in the $A_{+/ \times}(t)$ functions and in particular that the signal is fully described by its Fourier components at the five angular frequencies centered at the intrinsic angular frequency of the source, $\omega_{0}, \omega_{0} \pm \Omega, \omega_{0} \pm 2 \Omega$, where $\Omega$ is Earth's sidereal angular frequency. These five-component complex vectors identify the so-called 5-vector space onto which interferometric data can be projected. Let us call $\boldsymbol{X}$ and $\boldsymbol{A}_{+/ \times}$the 5-vectors for the data and the plus/cross polarization signal templates, respectively. The scalar products between $\boldsymbol{X}$ and $\boldsymbol{A}_{+/ \times}$ correspond to the matched filters between the data and the signal templates, and if opportunely normalized, these two quantities are the estimators of the signal plus and cross amplitudes

$$
\widehat{H}_{+/ \times}=\frac{\boldsymbol{X} \cdot \boldsymbol{A}_{+} / x}{\left|\boldsymbol{A}_{+/ \times}\right|^{2}},
$$

from which a detection statistic can be derived as

$$
\mathcal{S} \equiv\left|\boldsymbol{A}_{+}\right|^{4}\left|\widehat{H}_{+}\right|^{2}+\left|\boldsymbol{A}_{\times}\right|^{4}\left|\widehat{H}_{\times}\right|^{2} .
$$

We can use the value of this detection statistic to compute the associated significance and the false-alarm probability of a given candidate. To do so, we need to estimate also the noise background distribution, by repeating the calculation of $\mathcal{S}$ in an "off-source" analysis (far from the signal frequency). In the veto step described in Appendix A.1.3, we compute the statistical properties of the data (and noise) over chunks of data 


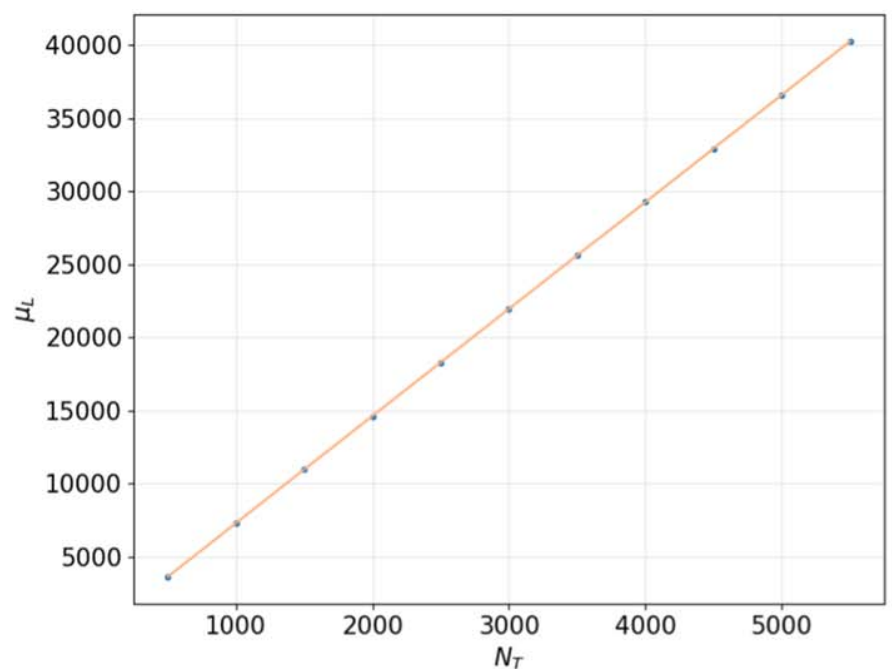

(a)

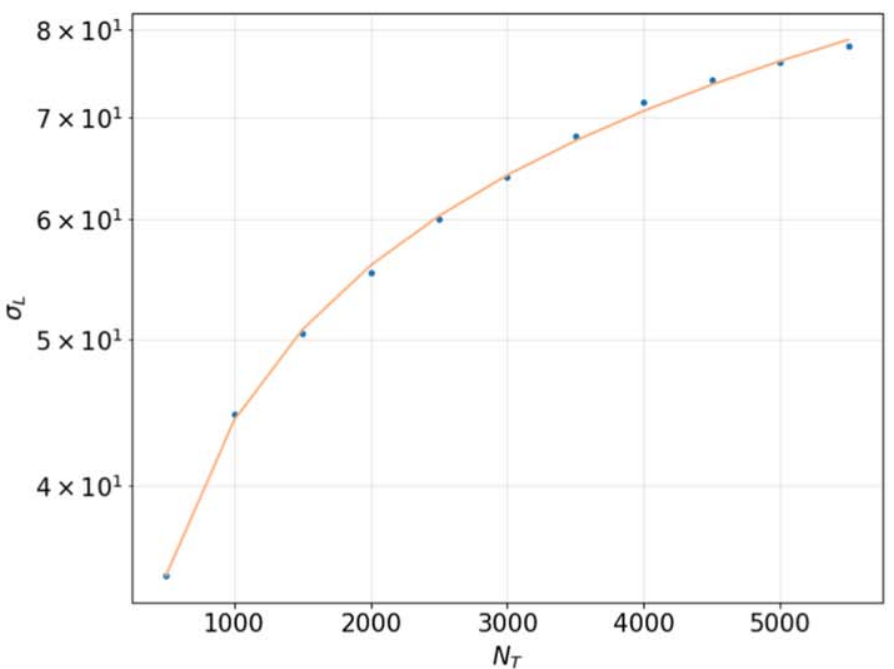

(b)

Figure 9. (a) Mean $\mu_{\mathcal{C}}$ and (b) standard deviation $\sigma_{\mathcal{L}}$ of the log-likelihoods of the Viterbi paths as a function of $N_{T}$. Blue circles are values obtained from 100 trials of simulations. The orange curves are the linear and power-law fits describing the mean and standard deviation dependence on $N_{T}$. An analogous calibration is presented in Millhouse et al. (2020).

of duration $T_{\text {sid }}$, by summing up the values of the statistic in each iteration. In this step, we compare the statistical features of the data twice: first using a time series corrected for the Doppler and the spin-down parameters provided by the candidate, and then using no correction. We expect that even if the correction is not precise, if the candidate is of astrophysical origin, the significance with respect to the uncorrected case will be higher. The same comparison is repeated using data of longer duration (4 times longer), which should correspond to an increase of the candidate significance proportional to the fourth square root of the coherence time used.

\section{B.2. Single-harmonic Viterbi}

In this section, we outline the methods used to determine the parameter space and detection thresholds used in the singleharmonic Viterbi pipeline.

First, we outline the process to determine the frequency range and $T_{\text {coh }}$ for each source. We determine the maximum and minimum spin-down rate $\dot{f}_{0}$ expected for the source assuming a typical signal model with $f=2 f_{\star}$,

$$
-\frac{f}{\left(n_{\min }-1\right) t_{\mathrm{age}}} \leqslant \dot{f}_{0} \leqslant-\frac{f}{\left(n_{\max }-1\right) t_{\mathrm{age}}},
$$

where $t_{\text {age }}=f_{\star} /\left[(n-1) \dot{f}_{\star}\right]$ and $n$ is the braking index. Because the braking index is unknown for each source, we use the most extreme plausible values $n_{\min }=2$ and $n_{\max }=7$. Also, we neglect stochastic spin wandering when determining the maximum $\dot{f}$ because we expect the spin-down rate to be much faster than the rate of spin wandering, especially in young SNRs. Using the maximum value of $\dot{f}_{0}^{\max }$ from Equation (B8), we make our first estimate of the coherence time

$$
T_{\text {coh }}=2^{-1 / 2}\left|\dot{f}_{0}^{\max }\right|^{-1 / 2}
$$

and calculate the analytically estimated sensitivity $h_{0}^{\text {est }}$ using Equation (6). We also calculate the inferred $h_{0}^{\max }$ using Equation (5). The initial estimate of $T_{\text {och }}$ is used only to find the
Table 7

The Threshold $\mathcal{L}_{\text {th }}$ for Each SNR in the Single-harmonic Viterbi Search

\begin{tabular}{lc}
\hline \hline Source & $\mathcal{L}_{\text {th }}$ \\
\hline G1.9+0.3 & 32552 \\
G15.9+0.2 & 32575 \\
G18.9-1.1 & 17417 \\
G39.2-0.3 & 11683 \\
G65.7+1.2 & 7160 \\
G93.3+6.9 & 17174 \\
G111.7-2.1 & 32568 \\
G189.1+3.0 & 24187 \\
G266.2-1.2 & 32579 \\
G291.0-0.1 & 32586 \\
G330.2+1.0 & 30130 \\
G347.3-0.5 & 32589 \\
G350.1-0.3 & 32577 \\
G353.6-0.7 & 3334 \\
G354.4+0.0 & 32553 \\
\hline
\end{tabular}

frequency range, with the maximum frequency covered in the search, $f_{\max }$, set to the maximum frequency for which $h_{0}^{\max }>h_{0}^{\text {est }}$. We then recalculate $T_{\text {coh }}$ using $f_{\max }$ to identify the $T_{\text {coh }}$ necessary to track the $\dot{f}_{0}^{\max }$ implied by $f_{\max }$ in Equation (B9). If, after recalculating $T_{\text {coh }}$, we have $T_{\text {coh }}<1 \mathrm{hr}$, we recalculate $\dot{f}_{\max }$ (by inverting Equation (B9)) using $T_{\text {coh }}=1 \mathrm{hr}$ and insert the new $\dot{f}_{\max }$ into Equation (B8) to obtain a new $f_{\max }$. We do not search using $T_{\text {coh }}<1 \mathrm{hr}$ because to do so would require reproducing and cleaning short Fourier transforms (SFTs) explicitly for this search, instead of using the same standard SFTs as other pipelines. In addition, using coherent time shorter than an hour would significantly degrade the sensitivity. If, after recalculating $T_{\text {coh }}$, we have $T_{\text {coh }} \geqslant 1 \mathrm{hr}$, we do not need to recalculate $f_{\max }$ and $\dot{f}_{0}^{\max }$. Finally, we determine the minimum search frequency $f_{\min }$ that satisfies $h_{0}^{\max }>h_{0}^{\text {est }}$. The values $T_{\text {coh }}$, $f_{\min }, f_{\max }$, and $\dot{f}_{0}^{\max }$ define the parameter space for the search and are summarized in Table 3.

Next, we outline the process of setting the detection threshold for each source. In each subband, the Viterbi algorithm obtains $N_{Q}$ 
frequency paths (ending in $N_{Q}$ different frequency bins), each with a $\log$-likelihood $\mathcal{L}$. We set a $\log$-likelihood threshold to determine which, if any, of the $N=N_{Q} N_{\text {band }}$ paths warrant further analysis. We require a false-alarm probability $\alpha_{N}=0.01$ for each source across all subbands. This is equivalent to requiring a false-alarm probability per subband of

$$
\alpha_{F}=1-\left(1-\alpha_{N}\right)^{1 / N} .
$$

The likelihood threshold $\mathcal{L}_{\text {th }}$ is then determined by solving

$$
\alpha_{F}=\int_{\mathcal{L}_{\text {th }}}^{\infty} \mathrm{d} \mathcal{L} p(\mathcal{L}) .
$$

The threshold $\mathcal{L}_{\text {th }}$ is unique to each source. We follow up any path with $\mathcal{L}>\mathcal{L}_{\text {th }}$.

While the distribution of the log-likelihoods is unknown (see Suvorova et al. 2016 for details), Millhouse et al. (2020) demonstrated that the mean $\mu_{L}$ and standard deviation $\sigma_{L}$ depend only on $N_{T}$ and scale according to linear and power-law relationships, respectively. We determine the form of these relationships by simulating 100 subbands of Gaussian noise for 11 different $N_{T}$ values in the range $500 \leqslant N_{T} \leqslant 5500$ and conduct a search on each band. From the log-likelihoods for each Viterbi path we calculate $\mu_{\mathcal{L}}, \sigma_{\mathcal{L}}$, and scaling relations of the log-likelihood distribution for each $N_{T}$. Figure 9 displays $\mu_{\mathcal{L}}$ and $\sigma_{\mathcal{L}}$ from simulations (blue circles) and from the scaling relations (orange curves).

For each source, we use the scaling relations from Figure 9 to define a Gaussian log-likelihood distribution $p(\mathcal{L})$ for $N_{T}=T_{\text {obs }} / T_{\text {coh }}$ and solve $\alpha_{F}=\int_{\mathcal{L}_{\text {th }}}^{\infty} p(\mathcal{L})$ to obtain $\mathcal{L}_{\text {th }}$ (Table 7). We follow up all unique frequency paths with $\mathcal{L}>\mathcal{L}_{\text {th }}$ using the procedure described in Appendix A.

\section{B.3. Dual-harmonic Viterbi}

The HMM formulation in the dual-harmonic search is essentially the same as described in Section 4.2, with modifications detailed in Section 4.3. Here we briefly review the dual-harmonic formulation (Sun et al. 2019) and describe the settings used in this analysis.

We select a coherent time interval, $T_{\text {coh }}$, and assume that

$$
\left|\int_{t}^{t+T_{\mathrm{coh}}} d t^{\prime} \dot{f}_{\star}\left(t^{\prime}\right)\right|<\Delta f
$$

is always satisfied, where $\Delta f=1 /\left(4 T_{\text {coh }}\right)$ is the frequency bin size in the $\mathcal{F}_{1}$ output. We use $2 \Delta f=1 /\left(2 T_{\text {coh }}\right)$ as the frequency bin size when computing $\mathcal{F}_{2}$ such that the signal is expected to move at most one bin in the outputs of both $\mathcal{F}_{1}$ and $\mathcal{F}_{2}$ over each discrete time step, i.e., from one coherent time interval to the next. The log emission probability computed over each $T_{\text {coh }}$ interval is (Jaranowski et al. 1998; Sun et al. 2019)

$$
\begin{aligned}
\ln L_{o\left(t_{k}\right) q\left(t_{k}\right)}= & \ln P\left[o\left(t_{k}\right) \mid f_{i} \leqslant f_{\star}\left(t_{k}\right) \leqslant f_{i}+\Delta f\right] \\
& =\mathcal{F}_{1}\left(f_{i}\right)+\mathcal{F}_{2}\left(2 f_{i}\right),
\end{aligned}
$$

where $f_{i}$ is the frequency value in the $i$ th bin. In this analysis, we assume that the frequency evolution in these young sources is dominated by the secular spin-down of the star, and hence the transition probability matrix $A_{q_{j} q_{i}}$ becomes (Sun et al. 2019)

$$
A_{q_{i-1} q_{i}}=A_{q_{i} q_{i}}=\frac{1}{2}
$$

with all other entries being zero. A uniform prior $\Pi\left[q\left(t_{0}\right)\right]=$ $N_{Q}^{-1}$ is used.

\section{References}

Aasi, J., Abadie, J., Abbott, B., et al. 2013, PhRvD, 88, 102002 Aasi, J., Abbott, B., Abbott, R., et al. 2015b, ApJ, 813, 39

Aasi, J., Abadie, J., Abbott, B. P., et al. 2015a, CQGra, 32, 074001 Abadie, J., Abbott, B. P., Abbott, R., et al. 2010, ApJ, 722, 1504 Abbott, B., Abbott, R., Abbott, T., et al. 2019a, PhRvD, 99, 122002 Abbott, B. P., Abbott, R., Abbott, T. D., et al. 2017, PhRvD, 95, 082005 Abbott, B. P., Abbott, R., Abbott, T. D., et al. 2019b, PhRvX, 9, 031040 Abbott, B. P., Abbott, R., Abbott, T. D., et al. 2019c, ApJ, 879, 10 Abbott, B. P., Abbott, R., Abbott, T. D., et al. 2019d, PhRvD, 100, 122002 Abbott, B. P., Abbott, R., Abbott, T. D., et al. 2019e, ApJ, 875, 122 Abbott, B. P., Abbott, R., Abbott, T. D., et al. 2019f, PhRvD, 100, 024004 Abbott, R., Abbott, T. D., Abraham, S., et al. 2020, ApJ, 902, L21 Abbott, R., Abbott, T. D., Abraham, S., et al. 2021a, PhRvX, 11, 021053 Abbott, R., Abbott, T. D., Abraham, S., et al. 2021b, ApJL, 913, L27 Abbott, R., Abbott, T. D., Abraham, S., et al. 2021c, PhRvD, 103, 064017 Acernese, F., Agathos, M., Agatsuma, K., et al. 2015, CQGra, 32, 024001

Allen, G. E., Chow, K., DeLaney, T., et al. 2014, ApJ, 798, 82

Ambrocio-Cruz, P., Rosado, M., de la Fuente, E., Silva, R., \& Blanco-Piñon, A. 2017, MNRAS, 472, 51

Andersson, N. 1998, ApJ, 502, 708

Antonucci, F., Astone, P., D’Antonio, S., Frasca, S., \& Palomba, C. 2008, CQGra, 25, 184015

Ashton, G., Hübner, M., Lasky, P. D., et al. 2019, ApJS, 241, 27

Ashton, G., Jones, D., \& Prix, R. 2015, PhRvD, 91, 062009

Astone, P., Colla, A., D'Antonio, S., et al. 2014a, PhRvD, 89, 062008

Astone, P., Colla, A., D'Antonio, S., Frasca, S., \& Palomba, C. 2014b, PhRvD, 90, 042002

Astone, P., D’Antonio, S., Frasca, S., \& Palomba, C. 2010, CQGra, 27, 194016

Astone, P., Frasca, S., \& Palomba, C. 2005, CQGra, 22, 1197

Banagiri, S., Sun, L., Coughlin, M. W., \& Melatos, A. 2019, PhRvD, 100, 024034

Behnke, B., Papa, M. A., \& Prix, R. 2015, PhRvD, 91, 064007

Bejger, M., \& Królak, A. 2014, CQGra, 31, 105011

Beniwal, D., Clearwater, P., Dunn, L., Melatos, A., \& Ottaway, D. 2021, PhRVD, 103, 083009

Bondarescu, R., Teukolsky, S. A., \& Wasserman, I. 2009, PhRvD, 79, 104003 Borkowski, K. J., Gwynne, P., Reynolds, S. P., et al. 2017, ApJ, 837, L7

Borkowski, K. J., Reynolds, S. P., Williams, B. J., \& Petre, R. 2018, ApJL, 868, L21

Buikema, A., Cahillane, C., Mansell, G. L., et al. 2020, PhRvD, 102, 062003 Caride, S., Inta, R., Owen, B. J., \& Rajbhandari, B. 2019, PhRvD, 100, 064013 Cassam-Chenai, G., Decourchelle, A., Ballet, J., et al. 2004, A\&A, 427, 199 Cordes, J. M., \& Greenstein, G. 1981, ApJ, 245, 1060

Covas, P. B., \& Sintes, A. M. 2020, PhRvL, 124, 191102

Cutler, C. 2002, PhRvD, 66, 084025

Davis, D., Areeda, J. S., Berger, B. K., et al. 2021, CQGra, 38, 135014

Dergachev, V., \& Papa, M. A. 2020, PhRvL, 125, 171101

Dergachev, V., Papa, M. A., Steltner, B., \& Eggenstein, H.-B. 2019, PhRvD, 99, 084048

Dreissigacker, C., Prix, R., \& Wette, K. 2018, PhRvD, 98, 084058

Ferrand, G., \& Safi-Harb, S. 2012, AdSpR, 49, 1313

Fesen, R., \& Kirshner, R. 1980, ApJ, 242, 1023

Fesen, R. A., Hammell, M. C., Morse, J., et al. 2006, ApJ, 645, 283

Foster, T., \& Routledge, D. 2003, ApJ, 598, 1005

Frasca, S., Astone, P., \& Palomba, C. 2005, CQGra, 22, S1013

Fukuda, T., Yoshiike, S., Sano, H., et al. 2014, ApJ, 788, 94

Gaensler, B., Tanna, A., Slane, P., et al. 2008, ApJL, 680, L37

Glampedakis, K., \& Gualtieri, L. 2018, in The Physics and Astrophysics of Neutron Stars, ed. L. Rezzolla et al., Vol. 457 (Berlin: Springer), 673

Gnedin, O. Y., Yakovlev, D. G., \& Potekhin, A. Y. 2001, MNRAS, 324, 725

Goetz, E., Neunzert, A., Riles, K., et al. 2021, Tech. Rep. LIGO-T2100153, LIGO Laboratory

Gotthelf, E. V., Halpern, J. P., \& Alford, J. 2013, ApJ, 765, 58

Green, D. A. 2019, JApA, 40, 36

Haensel, P., Urpin, V. A., \& Yakovlev, D. G. 1990, A\&A, 229, 133

Harrus, I. M., \& Slane, P. O. 1999, ApJ, 516, 811

Harrus, I. M., Slane, P. O., Hughes, J. P., \& Plucinsky, P. P. 2004, ApJ, 603,152

Hobbs, G., Lyne, A., \& Kramer, M. 2010, MNRAS, 402, 1027

Ilovaisky, S. A., \& Lequeux, J. 1972, A\&A, 18, 169 
Isi, M., Mastrogiovanni, S., Pitkin, M., \& Piccinni, O. J. 2020, PhRvD, 102, 123027

Jaranowski, P., Królak, A., \& Schutz, B. F. 1998, PhRvD, 58, 063001

Jiang, B., Chen, Y., \& Wang, Q. D. 2007, ApJ, 670, 1142

Johnson-McDaniel, N. K., \& Owen, B. J. 2013, PhRvD, 88, 044004

Jones, D., \& Sun, L. 2021, PhRvD, 103, 023020

Jones, D. I. 2010, MNRAS, 402, 2503

Kargaltsev, O., Pavlov, G. G., Klingler, N., \& Rangelov, B. 2017, JPIPh, 83, 635830501

Karpova, A., Zyuzin, D., Danilenko, A., \& Shibanov, Y. 2015, MNRAS, 453,2241

Klochkov, D., Suleimanov, V., Pühlhofer, G., et al. 2015, A\&A, 573, A53

Knispel, B., \& Allen, B. 2008, PhRvD, 78, 044031

Kothes, R., Landecker, T., Reich, W., Safi-Harb, S., \& Arzoumanian, Z. 2008, ApJ, 687, 516

Landecker, T. L., Routledge, D., Reynolds, S. P., et al. 1999, ApJ, 527, 866

Lasky, P. D., \& Melatos, A. 2013, PhRvD, 88, 103005

Lazendic, J. S., Slane, P. O., Gaensler, B. M., et al. 2003, ApJL, 593, L27

Leahy, D. A., Ranasinghe, S., \& Gelowitz, M. 2020, ApJS, 248, 16

Lindblom, L., \& Owen, B. J. 2020, PhRvD, 101, 083023

Liseau, R., Lorenzetti, D., Nisini, B., Spinoglio, L., \& Moneti, A. 1992, A\&A, 265,577

Lovchinsky, I., Slane, P., Gaensler, B., et al. 2011, ApJ, 731, 70

Lower, M. E., Bailes, M., Shannon, R. M., et al. 2020, MNRAS, 494, 228

Mastrano, A., Melatos, A., Reisenegger, A., \& Akgün, T. 2011, MNRAS, 417, 2288

McClure-Griffiths, N. M., Green, A. J., Dickey, J. M., et al. 2001, ApJ, 551,394

Melatos, A., Douglass, J. A., \& Simula, T. P. 2015, ApJ, 807, 132

Melatos, A., \& Link, B. 2014, MNRAS, 437, 21

Middleton, H., Clearwater, P., Melatos, A., \& Dunn, L. 2020, PhRvD, 102, 023006

Millhouse, M., Strang, L., \& Melatos, A. 2020, PhRvD, 102, 083025

Ming, J., Papa, M. A., Singh, A., et al. 2019, PhRvD, 100, 024063

Moffett, D., Gaensler, B., \& Green, A. 2001, AIP Conference Proceedings, 565,333

Namkham, N., Jaroenjittichai, P., \& Johnston, S. 2019, MNRAS, 487, 5854

Owen, B. J. 2010, PhRvD, 82, 104002

Owen, B. J., Lindblom, L., Cutler, C., et al. 1998, PhRvD, 58, 084020

Papa, M. A., Ming, J., Gotthelf, E. V., et al. 2020, ApJ, 897, 22

Park, S., Kargaltsev, O., Pavlov, G. G., et al. 2009, ApJ, 695, 431

Parthasarathy, A., Shannon, R. M., Johnston, S., et al. 2019, MNRAS, 489,3810

Piccinni, O. J., Astone, P., D’Antonio, S., et al. 2018, CQGra, 36, 015008

Piccinni, O. J., Astone, P., D’Antonio, S., et al. 2020, PhRvD, 101, 082004

Potekhin, A. Y., Pons, J. A., \& Page, D. 2015, SSRv, 191, 239

Price, S., Link, B., Shore, S., \& Nice, D. J. 2012, MNRAS, 426, 2507

Quinn, B. G., \& Hannan, E. J. 2001, The Estimation and Tracking of Frequency, Vol. 9 (Cambridge: Cambridge Univ. Press)
Ranasinghe, S., Leahy, D., \& Tian, W. W. 2020, JHEPGC, 6, 9

Reed, J. E., Hester, J. J., Fabian, A., \& Winkler, P. 1995, ApJ, 440, 706

Reynolds, S. P., Borkowski, K. J., Green, D. A., et al. 2008, ApJL, 680, L41

Reynolds, S. P., Borkowski, K. J., Hwang, U., et al. 2006, ApJL, 652, L45

Riles, K. 2013, PrPNP, 68, 1

Riles, K. 2017, MPLA, 32, 1730035

Roger, R. S., Milne, D. K., Caswell, J. L., \& Little, A. G. 1986, MNRAS, 219, 815

Roy, S., \& Pal, S. 2013, ApJ, 774, 150

Roy, S., \& Pal, S. 2014, in ASI Conf Ser, Vol. 13, ed. A. Ray \& R. A. McCray, 263

Sasaki, M., Mäkelä, M. M., Klochkov, D., Santangelo, A., \& Suleimanov, V. 2018, MNRAS, 479, 3033

Shan, S. S., Zhu, H., Tian, W. W., et al. 2018, ApJS, 238, 35

Shannon, R. M., \& Cordes, J. M. 2010, ApJ, 725, 1607

Slane, P., Gaensler, B. M., Dame, T. M., et al. 1999, ApJ, 525, 357

Slane, P., Hughes, J. P., Temim, T., et al. 2012, ApJ, 749, 131

2020, SNRcat-High Energy Observations of Galactic Supernova Remnants, http://snrcat.physics.umanitoba.ca/index.php?

Steltner, B., Papa, M. A., Eggenstein, H.-B., et al. 2021, ApJ, 909, 79

Straal, S. M., \& van Leeuwen, J. 2019, A\&A, 623, A90

Su, Y., Chen, Y., Yang, J., et al. 2010, ApJ, 727, 43

Sun, L., Goetz, E., Kissel, J. S., et al. 2020, CQGra, 37, 225008

Sun, L., Melatos, A., \& Lasky, P. D. 2019, PhRvD, 99, 123010

Sun, L., Melatos, A., Lasky, P. D., Chung, C. T. Y., \& Darman, N. S. 2016, PhRvD, 94, 082004

Sun, L., Melatos, A., Suvorova, S., Moran, W., \& Evans, R. J. 2018, PhRvD, 97, 043013

Suvorova, S., Sun, L., Melatos, A., Moran, W., \& Evans, R. J. 2016, PhRvD, 93, 123009

Swartz, D. A., Pavlov, G. G., Clarke, T., et al. 2015, ApJ, 808, 84

Tian, W. W., Leahy, D. A., Haverkorn, M., \& Jiang, B. 2008, ApJL, 679, L85

Tsuji, N., \& Uchiyama, Y. 2016, PASJ, 68, 108

Ushomirsky, G., Cutler, C., \& Bildsten, L. 2000, MNRAS, 319, 902

van den Bergh, S. 1971, ApJ, 165, 457

Van Den Broeck, C. 2005, CQGra, 22, 1825

Viterbi, A. 1967, ITIT, 13, 260

Wang, Z. R., Qu, Q. Y., \& Chen, Y. 1997, A\&A, 318, L59

Wette, K., Dunn, L., Clearwater, P., \& Melatos, A. 2021, PhRvD, 103, 083020

Wette, K., Owen, B., Allen, B., et al. 2008, CQGra, 25, 235011

Wilson, E. B. 1927, Journal of the American Statistical Association, 22, 209

Woan, G., Pitkin, M. D., Haskell, B., Jones, D. I., \& Lasky, P. D. 2018, ApJ, 863, L40

Yasumi, M., Nobukawa, M., Nakashima, S., et al. 2014, PASJ, 66, 68

Zhang, Y., Papa, M. A., Krishnan, B., \& Watts, A. L. 2021, ApJ, 906, L14

Zhu, S. J., Papa, M. A., \& Walsh, S. 2017, PhRvD, 96, 124007

Zimmermann, M., \& Szedenits, E., Jr. 1979, PhRvD, 20, 351

Zweizig, J., \& Riles, K. 2020, Tech. Rep. LIGO-T2000384, LIGO Laboratory 